\title{
From the Solar Corona to Clusters of Galaxies: The Radio Astronomy of Bruce Slee
}

\author{
Wayne Orchiston \\ Australia Telescope National Facility, PO Box 76, Epping NSW 2121, Australia \\ (e-mail: Wayne.Orchiston@csiro.au)
}

Received 2003 May 8, accepted 2003 September 27

\begin{abstract}
Owen Bruce Slee is one of the pioneers of Australian radio astronomy. During World War II he independently discovered solar radio emission, and, after joining the CSIRO Division of Radiophysics, used a succession of increasingly more sophisticated radio telescopes to examine an amazing variety of celestial objects and phenomena. These ranged from the solar corona and other targets in our solar system, to different types of stars and the ISM in our Galaxy, and beyond to distant galaxies and clusters of galaxies. Although long retired, Slee continues to carry out research, with emphasis on active stars and clusters of galaxies. A quiet and unassuming man, Slee has spent more than half a century making an important, wide-ranging contribution to astronomy, and his work deserves to be more widely known.
\end{abstract}

Keywords: biographies — Bruce Slee — radio continuum: galaxies — radio continuum: ISM — radio continuum: stars — stars: flare — Sun: corona

\section{Introduction}

Radio astronomy is so new a discipline that it has yet to acquire an extensive historical bibliography. With foundations dating from 1931 this is perhaps not surprising, and it is only since the publication of Sullivan's classic work, The Early Years of Radio Astronomy, in 1984 that scholars have begun to take a serious interest in the history of this discipline and its 'key players'. However, in this context, the prior appearance of Serendipitous Discoveries in Radio Astronomy (Kellermann and Sheets, 1983), with its focus on Karl Jansky, deserves special mention.

Australia has long been a leader in radio astronomy (Sullivan, 1988), but apart from the publication of a special issue of the Australian Journal of Physics devoted to the late John Bolton (Goddard and Haynes, 1994), there has been little attempt to document the achievements of some of our most notable radio astronomers. This has become a pressing issue as most of these people retired years ago, and many are no longer with us. This particular study focusses on one of our pioneers, a man who began to hone his scientific skills in 1946 - in the formative days of Australian radio astronomy. He is now long retired, but continues an active research program. I first had the pleasure of working with him in 1961, and although quiet and unassuming, he has been an inspiration to me - and to others. His name is Bruce Slee.

Owen Bruce Slee (Fig. 1) was born on 1924 August 10 in Adelaide (for localities mentioned in the text see Fig. 2). Bruce was the second of three children, and his father worked as a carpenter. During the Great Depression life was particularly difficult. The family eventually lost their home, and went to live with relatives at Kadina

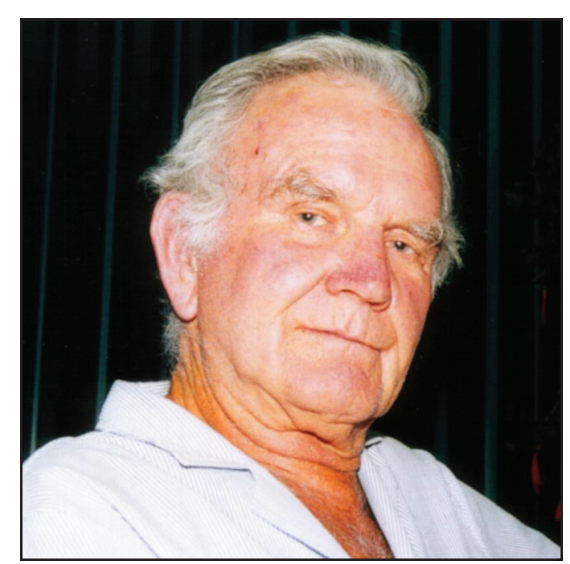

Figure 1 Owen Bruce Slee (1924-) in 2002.

on the Yorke Peninsula, where Slee's father worked at a gold mine. He followed this with another mining position at Woodside in the Adelaide Hills, the family moving there in 1936. After completing his primary schooling at Woodside, Slee attended secondary school in nearby Birdwood, where he proved to be an able student. Armed with good grades in the Leaving Certificate examinations, Slee then accepted a clerical job in the Lands Department, and one year later, at the age of 18 , he joined the Royal Australian Air Force.

Aircraftsman Slee was sent to Melbourne to attend a six-month radio mechanics course, and was then selected to complete a three-month radar mechanics course at Richmond Air Force Base. He was then posted to a radar station in Albany, Western Australia, and about one year later moved to another radar unit, at Onslow. His third 


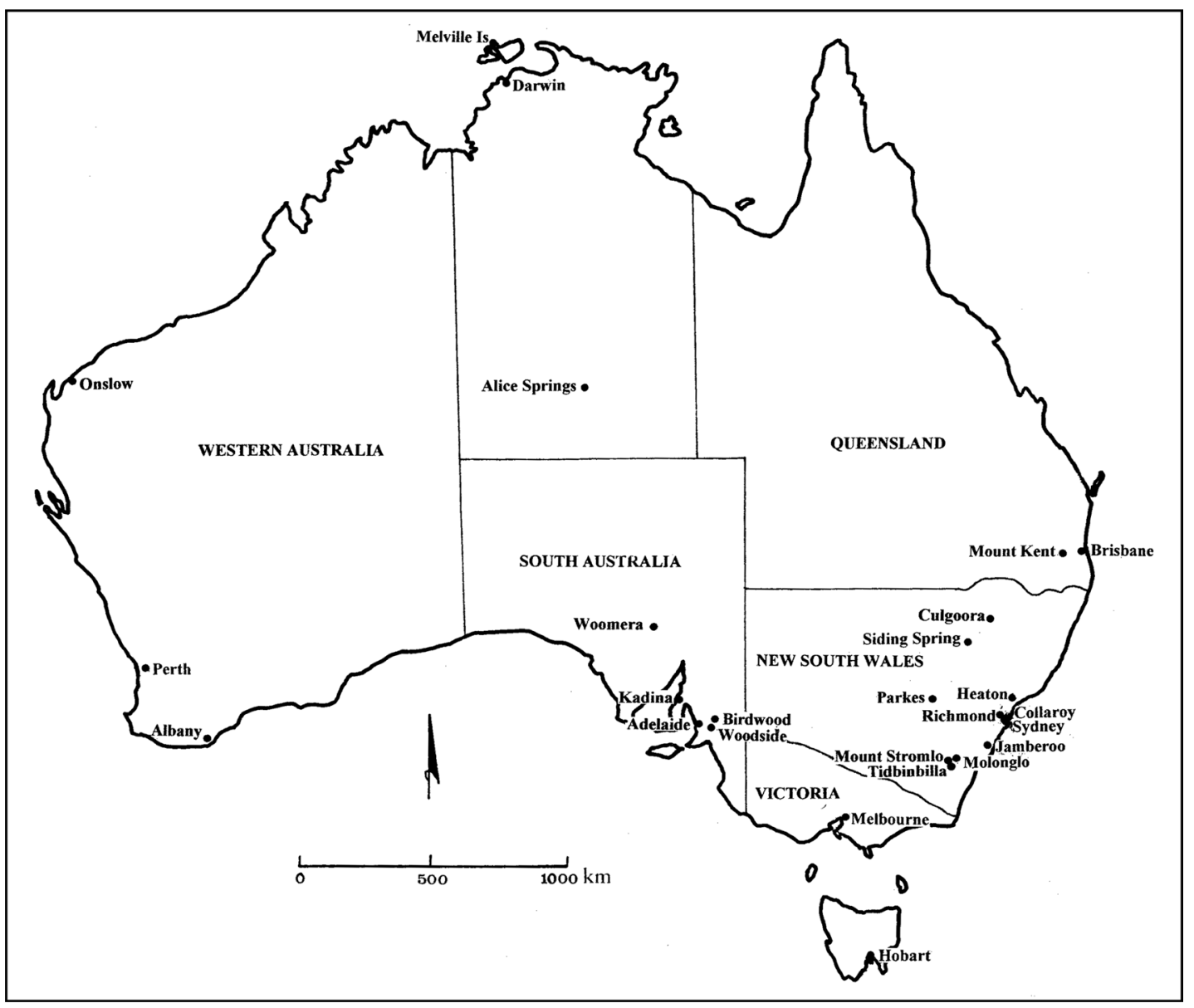

Figure 2 Australian localities mentioned in the text.

posting was to a radar station on the northern tip of Melville Island, and several months later he transferred to Radar Station 59 on the mainland, near Darwin, eventually becoming officer-in-charge with a rank of sergeant. It was there, during the second half of 1945, that he noticed there was sometimes distinctive 'static' when the antenna was pointing west near the time of sunset. By driving the antenna in azimuth, he noted that the static increased when the Sun was in the beam and concluded that this 'interference' was in fact solar radio emission (see Orchiston and Slee, 2002a). Slee did not publish this discovery, but what he did do was submit a report on this work to the CSIRO's Division of Radiophysics in Sydney.

As it happened, Slee's detection of $200 \mathrm{MHz}$ radio emission occurred soon after other independent war-time detections made by Reber and Southworth in the USA, German radar operators in Denmark, Hey in England and Alexander in New Zealand (see Orchiston, 1994b; Schott, 1947; Sullivan, 1984). The Division of Radiophysics was already aware of this emission, having received 'Top Secret' reports from England and New Zealand, and from October 1945 staff from the Division carried out successful solar observations using an antenna located at the Collaroy radar station near Sydney (see Pawsey, 1946). Fortuitously, there was a particularly active sunspot group present at this time, which was undoubtedly responsible for the emission detected by the Sydney radio astronomers and by Slee. Further successful observations were also made at this time in New Zealand.

After the War, Slee returned to his Lands Department job in Adelaide, and was subsequently contacted by Dr Joe Pawsey, head of the radio astronomy group at the Division of Radiophysics, who wrote about forthcoming vacancies in the Division. Pawsey visited Adelaide in 1946 August, interviewed Slee, was suitably impressed, and invited him to join the group. Slee accepted this invitation and in 1946 November moved to Sydney and was immediately posted to the Dover Heights field station as John Bolton's technical assistant. Apart from a brief interlude at the short-lived Georges Heights field station, assisting Don Yabsley with solar observations using an ex-WWII radar unit (Fig. 3), he remained at Dover Heights until transferring to Fleurs in 1954 when this field station opened.

Soon after joining the Division of Radiophysics, Slee began a five-year Diploma in Radio Engineering at Sydney Technical College and followed this with a two-year Diploma in Physics. Juggling these evening studies with his daytime employment and a growing family (for by 1952 he and Nan had two young daughters) was a challenge, but he succeeded admirably. Armed with assorted 


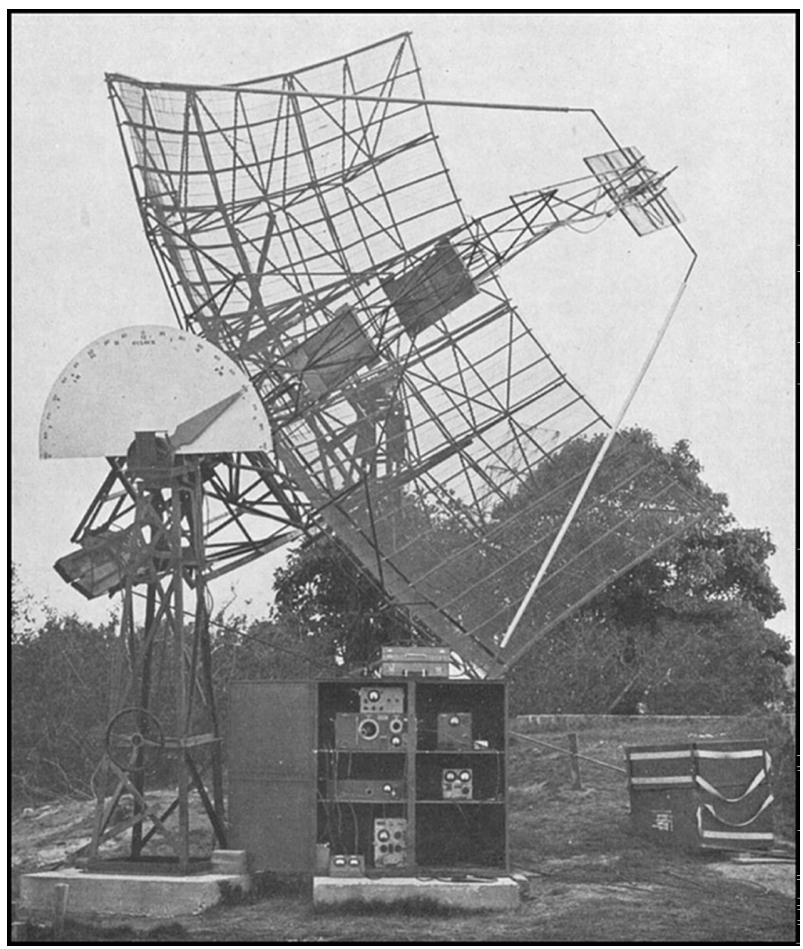

Figure 3 The ex-WWII radar antenna at Georges Heights field station that was used for solar observations in 1947 (ATNF Historic Photographic Archive).

credits from both Diploma courses, he began a part-time B.Sc. degree at the University of New South Wales, graduating with first-class honours in 1959.

By 1961 Slee had moved up the ranks at Radiophysics and was a research officer, and it was at this time that I joined him at Fleurs, serving as his technical assistant. When Fleurs field station closed in 1963, Slee returned to the Radiophysics Laboratory and continued his research programs from there, initially by means of the 64-m Parkes radio telescope. His wide-ranging research saw a continual flow of research papers, and in 1971 these were brought together for a D.Sc., which was awarded by the University of New South Wales.

Apart from a two-year sojourn in England during the 1960s, Slee spent the remainder of his working life with the Division of Radiophysics and its successor, the Australia Telescope National Facility (ATNF). By the time he retired as a principal research scientist in 1989, he had witnessed an amazing change in the nature of radio astronomical research. Back in the 1940s and 1950s, instrumentation had been simple and relatively inexpensive, and most of the emphasis was on the Division's scattered field stations. Christiansen (1984: 113, 115) paints a poignant picture of these halcyon days:

"The fieldwork had a pioneering appearance. Each morning people set off in open trucks to the field stations where their equipment, mainly salvaged and modified from radar installations, had been installed in ex-army and navy huts. At the field stations the atmosphere was completely informal and egalitarian, with dirty jobs shared by all. Thermionic valves were in frequent need of replacement and old and well-used co-axial connectors were a constant source of trouble. All receivers suffered from drifts in gain and 'system-noise' of hundreds or thousands of degrees represented the state of the art. During this period there was no place for observers who were incapable of repairing and maintaining the equipment. One constantly expected trouble."

The opening of the Parkes Radio Telescope in 1961 marked a new era in Australian radio astronomy, one associated with 'big science', which culminated more than two decades later with the construction of the Australia Telescope (see Haynes, Haynes, Malin and McGee, 1996; Robertson, 1992). Gone were radio telescopes that could be constructed and maintained by a single individual or a small group of colleagues. Gone too was the chance to engage in long-term projects involving extended observing time. As the focus of international radio astronomy shifted from the solar system to the far reaches of the universe, observing time tended to be assigned in short discrete blocks, and international collaborations became the norm. Papers with ten, twenty and sometimes more co-authors were not uncommon.

Since his retirement, Slee has continued his research programs as a research associate of the ATNF, and this is his story, a personal saga that began in the infant days of radio astronomy, spans more than half a century and a succession of Radiophysics field stations, and takes us from the simple Yagis that made up the early sea interferometers at Dover Heights, past the Mills Cross, the Parkes Radio Telescope, the Culgoora Radioheliograph to the Australia Telescope Compact Array (ATCA) and the Very Large Array (VLA). It is a journey that ranges from the solar corona, past Jupiter and Io and the comets of our solar system, out to the stars, pulsars and nebulae in our Galaxy, and beyond to distant quasars and clusters of galaxies.

\section{Research Programs}

\subsection{Investigating the Enigmatic 'Radio Stars'}

Upon his appointment to the Division of Radiophysics, Slee was assigned to John Bolton as his technical assistant, and spent his initial month working at the Dover Heights field station. This 5-ha facility in the eastern suburbs of Sydney was located on a 79-m cliff top overlooking the Tasman Sea, $5 \mathrm{~km}$ south of the entrance to Sydney Harbour, and the site offered two concrete blockhouses, a WWII radar antenna and direct access to water and power. Dover Heights was merely one of nine scattered field stations in the Sydney region that were maintained by the Division during the 1940s and/or 1950s as Figure 4 indicates.

Bolton and Slee's initial project at Dover Heights was to investigate solar bursts with a simple sea interferometer, but the Sun was inactive at the time and Bolton quickly tired of this. He decided instead that they would search for the enigmatic 'radio star' in Cygnus recently reported 
by Hey, Phillips and Parsons (1946). These observations, too, were unsuccessful, but

“... after a week or two they were cut short by an unheralded visit from Pawsey, who noted that the aerials were not looking at the Sun. Suffice it to say that he was not amused and we were both ordered back to the Lab. Bruce was reassigned to McCready to work on receiver construction and I to assist Gordon Stanley, who was building equipment to go on an eclipse expedition the following year." (Bolton, 1982: 349-350).

For Slee, this was hardly an auspicious start to a lifetime of involvement in radio astronomy!

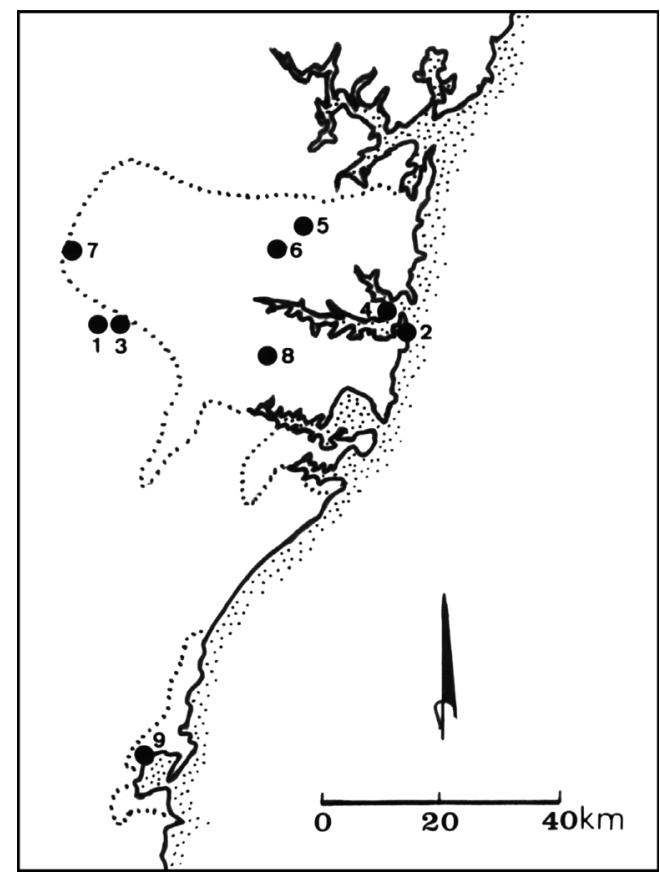

Figure 4 Major radio astronomy field stations in and near Sydney, 1945-1960. (Key: 1 = Badgerys Creek, $2=$ Dover Heights, $3=$ Fleurs, $4=$ Georges Heights, $5=$ Hornsby Valley, $6=$ Murray bank, $7=$ Penrith, $8=$ Potts Hill, and $9=$ Dapto; the dotted boundaries indicate the present-day Greater Sydney and Wollongong regions.) At various times, Slee worked at locations 2, 3 and 4.
When the Brazilian eclipse expedition was cancelled, Bolton was able to return to Dover Heights in league with Stanley, and after some initial solar monitoring they then spent the next three months observing Cygnus A at 60, 85,100 and $200 \mathrm{MHz}$ using simple Yagi antennas operating as sea interferometers (see Bolton and Slee, 1953, for a discussion of the sea interferometer principle). Slee joined them in 1947 September, in order “... to assist with improvements to receivers and antennas in preparation for a search for weaker sources at $100 \mathrm{MHz}$." (Slee, 1994: $520)$. The three then began a study of radio stars that would evolve into a long-term multi-faceted research program and one of the mainstays of the Division. And it was this program which opened up a whole new field of investigation, one that has remained central to the development of radio astronomy through to the present day (see Sullivan, 1982: 221).

On 1947 November 6, Bolton, Stanley and Slee detected a second source, Taurus A (Fig. 5), and within the next two months five additional sources. In a paper in Nature reporting these discoveries, Bolton (1948) commented on the difficulties associated with obtaining precise locations, and also noted that as in the case of Cygnus, the new sources did not appear to be associated with any notable stellar objects. The true nature of 'radio stars' would only be revealed when precise source positions were available, and so Bolton and Stanley arranged a field trip to New Zealand where the risings and settings of the sources could be observed without interruption, and from coastal sites with greater elevations than Dover Heights that therefore would provide higher-resolution interference fringes. From 1948 June, Bolton and Stanley spent three months in northern New Zealand observing Cygnus A, Taurus A, Centaurus A and Virgo A at $100 \mathrm{MHz}$ with a simple mobile 4-Yagi sea interferometer, while Slee continued to make parallel observations from Dover Heights. The New Zealand fieldtrip was a resounding success (Orchiston, 1993, 1994a), and in a paper published in Nature, Bolton, Stanley and Slee (1949) associated Taurus A with the Crab Nebula, Centaurus A with NGC 5128 and Virgo A with NGC 4486. This, incidentally, was Slee's first

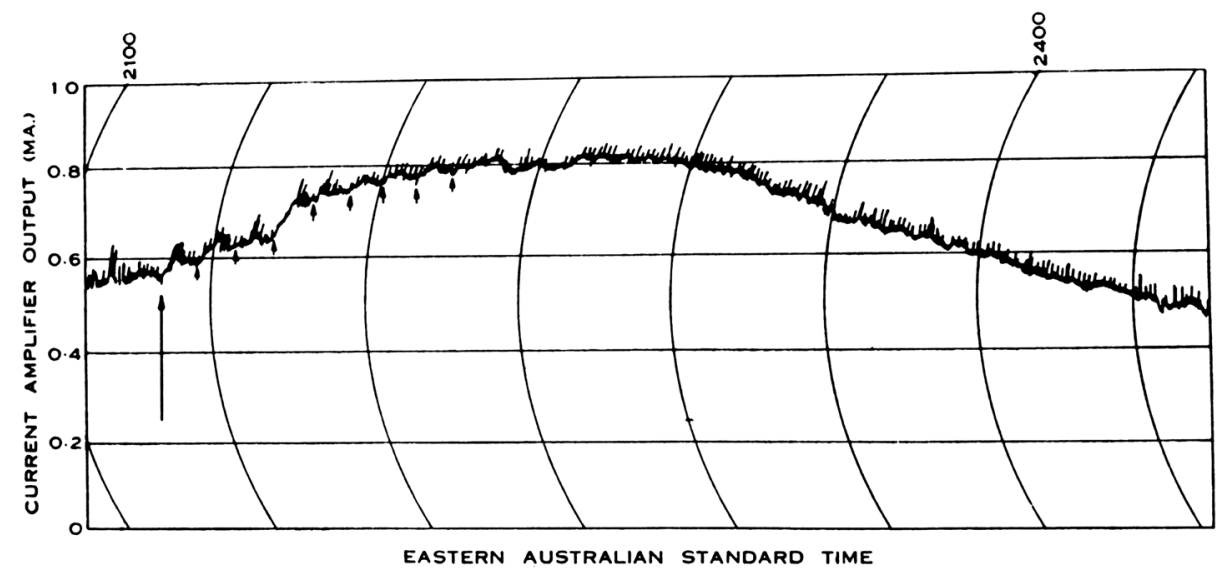

Figure 5 Chart record showing the discovery of the Taurus A source. The interferometer fringe pattern is indicated by the arrows (after Bolton and Stanley, 1949: 140). 
scientific paper, just as he was about to celebrate his 25 th birthday. Now only the identity of Cygnus A was in doubt, and although the New Zealand position of R.A. $19^{\mathrm{h}} 58^{\mathrm{m}}$ $14^{\mathrm{s}}$ and Dec. $+40^{\circ} 36^{\prime}$ was an improvement on the Dover Heights value (Stanley and Slee 1950), it would take several more years and even more precise positions before an optical correlate would emerge (see Bolton, 1955).

One other interesting outcome of the New Zealand field trip was a resolution of the enigmatic intensity fluctuations that were a conspicuous feature of Cygnus A. Simultaneous trans-Tasman observations showed that these were clearly due to scintillations, probably of ionospheric origin, and were not intrinsic to the source. Stanley and Slee (1950: 246) noted that intensity fluctuations also were associated with several other sources, particularly Centaurus A.

After returning from New Zealand and while analysing the newly acquired data, Bolton, Stanley and Slee continued their Dover Heights source survey, and by the end of 1948 had discovered seven new discrete sources.

Early in 1949 staff from the Radiophysics Laboratory Workshop installed an equatorially-mounted 9-Yagi array on top of the blockhouse at Dover Heights. Operating at $100 \mathrm{MHz}$, and with a $17^{\circ}$ beam, this new antenna was used by Bolton, Stanley, Slee and Westfold to survey Galactic radio emission between $140^{\circ}<l<40^{\circ}$ and $|b| \leq 60^{\circ}$, an area of the sky that included the galactic centre and was largely inaccessible from the northern hemisphere (see Bolton and Westfold 1950). In addition to producing a plot of the all-sky distribution of $100-\mathrm{MHz}$ radio emission, the array, in sea-interferometer mode, also revealed six new discrete sources. As a result, Stanley and Slee (1950) were able to list 18 different sources detected at Dover Heights between 1946 and 1949. They also included plots of the first radio spectra of Cygnus A, Virgo A, Centaurus A and Taurus A.
With Bolton and Westfold both overseas in 1950, Stanley and Slee were left to carry on the radio astronomy at Dover Heights, and Slee used observations of Cygnus A, Taurus A, Virgo A and Centaurus A to continue his systematic study of source scintillations. This was his first independent research project. He and Stanley also constructed a 3.9-m parabolic dish that eventually was mounted on the roof of the blockhouse and was used to observe the stronger sources at frequencies between 100 and $400 \mathrm{MHz}$ in order to investigate their spectra (Bolton 1982; Stanley 1994), but no searches for new sources were carried out.

A new source survey only became possible in 1951, after Bolton's return from overseas, when a new $4 \times 2$ Yagi array was constructed by Radiophysics Workshop staff, and installed on an ex-WWII radar altazimuth mount at Dover Heights. Soon after, this antenna was extended to a $6 \times 2$-Yagi array, still on the same mount (Fig. 6).

During 1951 and 1952, Bolton, Stanley and Slee used this new array to carry out what would prove to be the last major source survey at Dover Heights, and by far the most comprehensive one. With the benefit of improved electronics and a beamwidth of just $12^{\circ}$, this survey produced 104 sources. Bolton, Stanley and Slee, in various authorship combinations, then prepared three different papers on aspects of this survey, but often incorporating data from the earlier Dover Heights source surveys (see Bolton, Stanley and Slee, 1953; Bolton, Stanley and Slee, 1954; and Bolton, Westfold, Stanley and Slee, 1954). Slee also made a special study of the intensity variations exhibited by the Hydra A source, and this was written up as his first solo paper (Slee, 1955; c.f. Bolton and Slee, 1957).

One other interesting Dover Heights development relating to discrete sources that should be mentioned was the construction of a prototype two-element azimuth

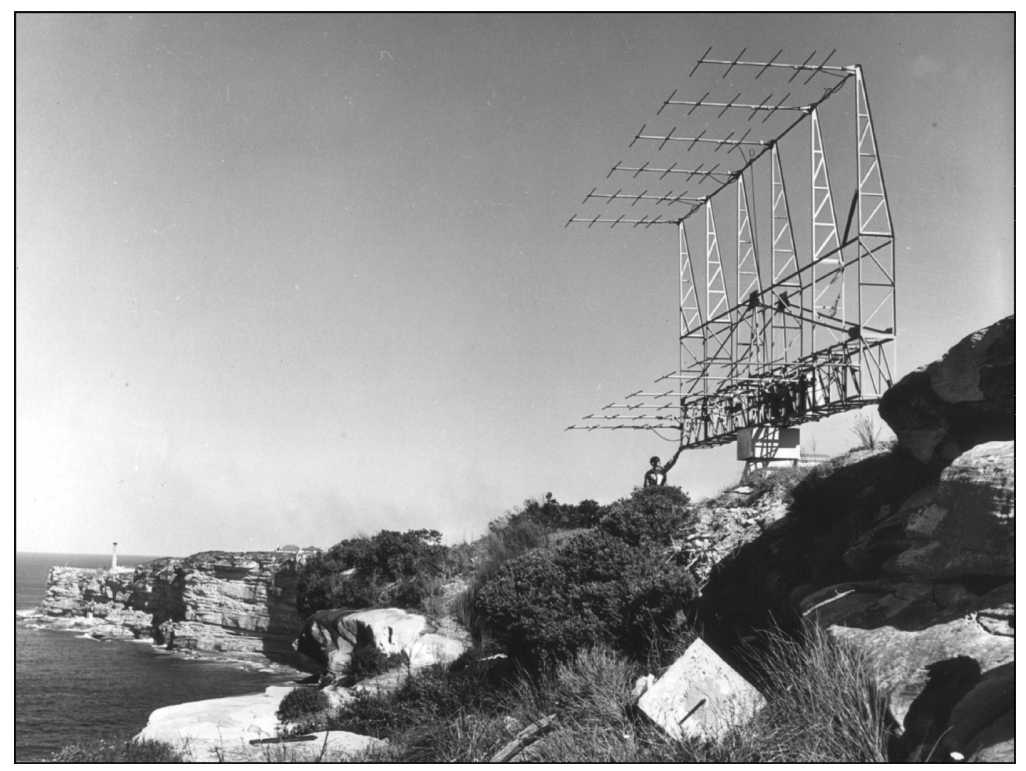

Figure 6 The $6 \times 2$-Yagi array, used for the most comprehensive Dover Heights source survey (Photograph: ATNF Historic Photographic Archive, 2763-8). 


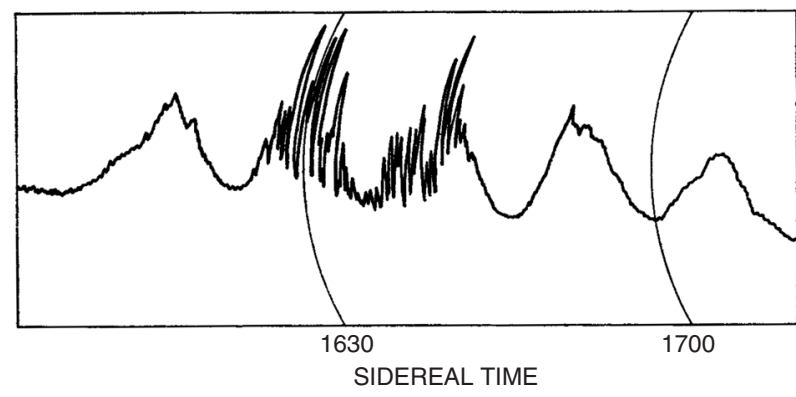

Figure 7 Chart record of Cygnus A showing scintillations (after Bolton, Stanley and Slee, 1953: 436).

interferometer in 1951. Bolton and Slee (1953: 431-432) explain the basics of this new system:

"In this system two aerials spaced along the cliff edge are used. The spaced aerials produce a second set of interference fringes at right angles to the normal sea interference fringes. The two aerials are connected to the receiver alternatively in and out of phase, thus switching the azimuth fringe system through half a fringe width. A synchronous rectifier at the receiver output detects only signals due to sources or irregularities in the background distribution smaller than the angular separation of the azimuth fringes."

Although the two Yagi arrays were spaced just $15 \mathrm{~m}$ apart, to their surprise Bolton, Stanley and Slee recorded many sources that were not seen with the sea interferometer. Amongst the sources detected was Puppis-A, and Baade and Minkowski (1954) were able to associate it with a supernova remnant. Although further research was carried out on some of the extended sources, no attempt was made to plot the numerous discrete sources that did not show up on the sea interferometer records.

With the completion of the $6 \times 2$-Yagi survey the days of the sea interferometer were over. Although it offered both advantages and disadvantages (see Stanley, 1994), it was soon replaced by spaced interferometers and cross arrays, bringing radio astronomy at Dover Heights to an end.

\subsection{Source Scintillations and the Ionosphere}

One of the early results to come from the Dover Heights radio stars project was the discovery that variations in the intensity of Cygnus-A and other 'radio stars' were not intrinsic to the source itself but were associated with scintillations (Bolton and Slee, 1957; Bolton, Slee and Stanley, 1953; Slee, 1955), and this was to open up a whole new area of investigation that Slee would pursue while based at the Fleur field station. An example of scintillations on the interferometer record of Cygnus A on 1948 May 19 is shown in Figure 7.

Irregularities in the Earth's ionosphere were seen as the cause of scintillations, but while Hewish concluded these were located at a height of $\sim 400 \mathrm{~km}$, Booker believed they were at a much lower level, between the $E$ and $F$ regions. In 1958 February-March, Slee (1958) recorded the signal transmitted by Satellite $1958 \alpha$ at $108 \mathrm{MHz}$, and his results supported Booker's views. Smerd then joined Slee in examining variations in Fleurs radio source scintillations with season, time of day and solar distance, and produced compelling evidence for the ionospheric origin of scintillations (Smerd and Slee, 1966).

Slee was also interested in the relationship between Fleurs radio source scintillations and the Earth's magnetic field. Using a sample of radio sources observed with the Mills Cross between 1955 and 1959, he showed that a relationship existed between source scintillations and geomagnetic activity, but concluded that "... it is improbable that geomagnetic variation is a direct cause of widespread intense scintillation ... [rather] a scintillation mechanism already present is made more effective on magnetically disturbed nights ...." (Slee, 1962: 570).

Following the opening of the Culgoora Radioheliograph, Slee and Lee were able to determine regular refraction in the ionosphere near the peak of the 1968-1971 solar activity cycle by using 2000 measurements of source positions at $80 \mathrm{MHz}$. Their results can be summarised as follows:

"Diurnal, seasonal and zenith-angle dependencies of the refraction are deduced and found to be quantitatively consistent with the known gradients in electron density. The variability of the refraction from one measurement to the next is found to depend upon the zenith angle and season and to be mainly due to ionospheric disturbances whose presence is not usually revealed by measurements of the $F_{2}$ critical frequency.... [We find] a value of $190 \pm 12 \mathrm{~km}$ for the equivalent thickness of the nocturnal ionosphere. A comparison of the present measurement of the thickness parameter with measurements of the same quantity made during the minimum of solar activity shows that the shape of the $F_{2}$ layer is not dependent on the solar activity cycle and hence is not a sensitive measure of the gas temperature." (Slee and Lee, 1974: 838).

\subsection{Cosmology and the MSH Catalogue}

After leaving Dover Heights in 1954, Slee transferred to the new field station at Fleurs, which was destined to make a major contribution to radio astronomy (see Orchiston and Slee, 2002c). Fleurs eventually was home to three innovative cross-type radio telescopes, and the first of these, the Mills Cross (Fig. 8), was under construction in 1954, following the erection and testing of a prototype at the Potts Hill field station in 1953 (see Mills and Little, 1953).

The Mills Cross at Fleurs (Mills, Little, Sheridan and Slee, 1958) comprised north-south and east-west arms each $450 \mathrm{~m}$ in length, and containing two rows of 250 half-wave dipole elements backed by a plain wire mesh reflector. The array operated at $85.5 \mathrm{MHz}$, with a 50 arc minutes pencil beam and a sensitivity, under ideal conditions, of $\sim 2$ Jy. Effectively a transit instrument, pointing 


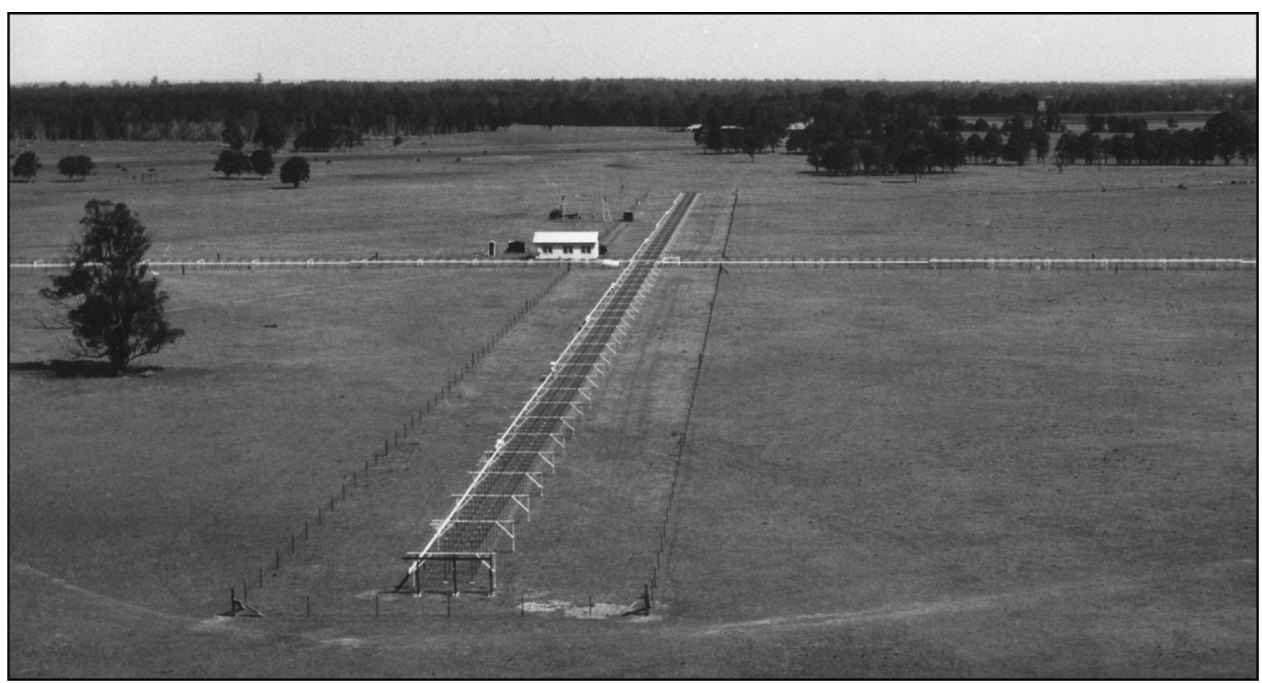

Figure 8 The newly constructed Mills Cross from the north looking along the north-south arm, showing the central receiver hut, and most of the east-west arm (ATNF Historic Photographic Archive, 3476-3).

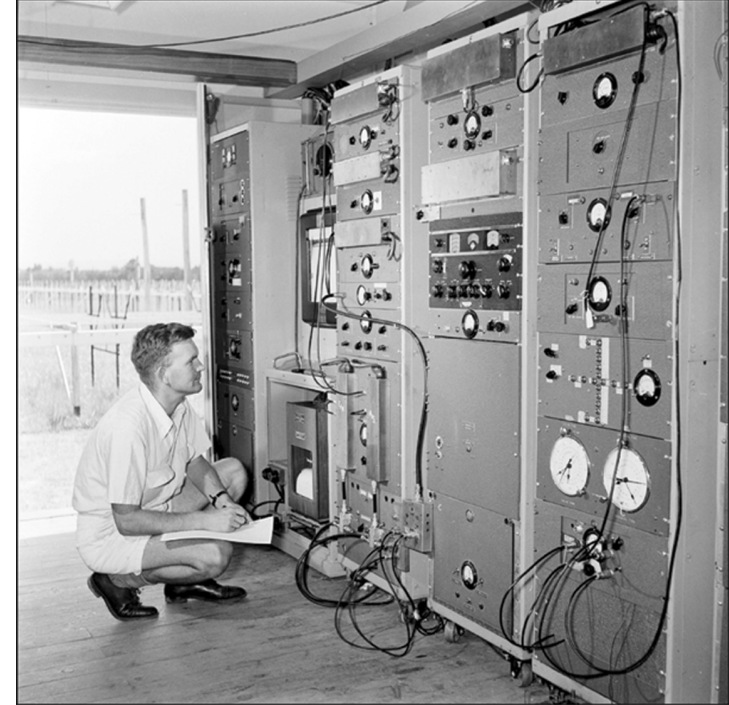

Figure 9 View showing Bruce Slee examining the chart recorders and Mills Cross receiver in 1955 (ATNF Historic Photographic Archive, 3868-10).

in declination could be achieved by altering the phases of the dipoles in the north-south arm. In this way, it was possible to survey the entire sky between declination $+10^{\circ}$ and $-80^{\circ}$ (corresponding to zenith distances of $+44^{\circ}$ and $-46^{\circ}$ respectively).

Armed with this new research instrument, Mills and Slee (Fig. 9) began a survey of the sky, obtaining their first results by end of 1954. The intention was to conduct an all-sky survey, and in order to speed up the observing program, five separate declinations 20 min of arc apart were scanned semi-simultaneously (see Fig. 10), even though this involved sacrificing some sensitivity and positional accuracy.

Initially, Mills and Slee (1957) produced a preliminary catalogue of sources in the region R.A. $00^{\mathrm{h}}$ to $08^{\mathrm{h}}$ and Dec. $+10^{\circ}$ to $-20^{\circ}$, in order to compare their results with

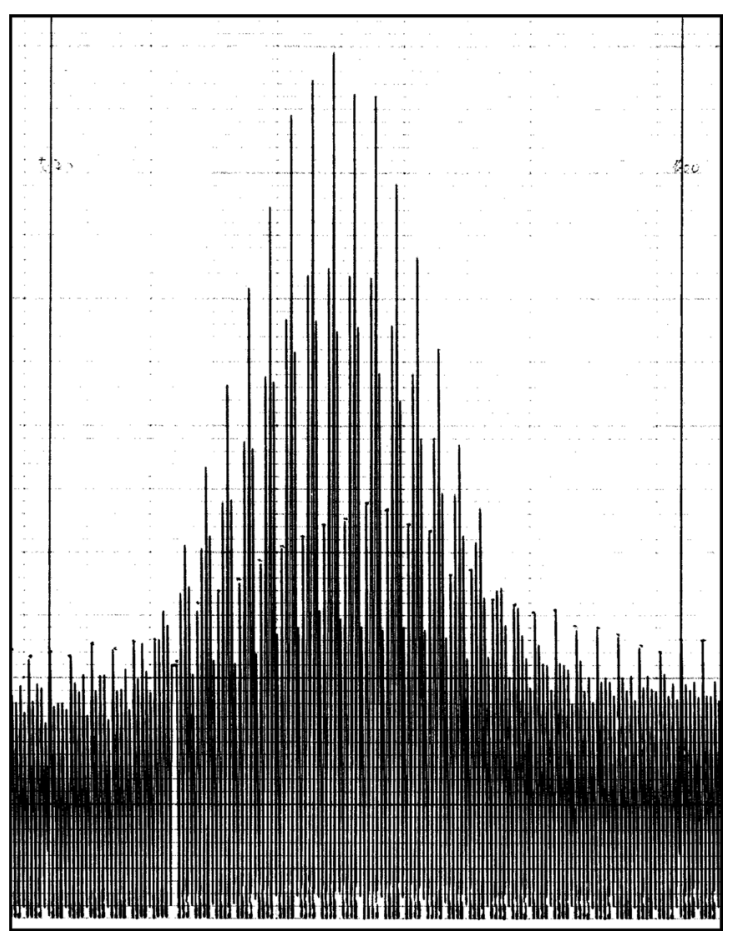

Figure 10 Example of a Mill Cross chart record showing the drift profile of a source recorded during all five declination scans.

those recently published by Shakeshaft et al. (1955). The outcome of their comparison was illuminating, as Mills (1984: 153) was later to write:

"My first realisation that all was not well with the new Cambridge survey came with a letter from Hoyle. He was worried about the results of counting sources catalogued in the $2 C$ survey, as reported by Ryle. For a homogeneous distribution of sources, not too far away, the cumulative $\log N-\log S$ count should have a slope of -1.5 . Ryle reported a slope of -3.0 and used this result to argue that the sources were placed at a great 


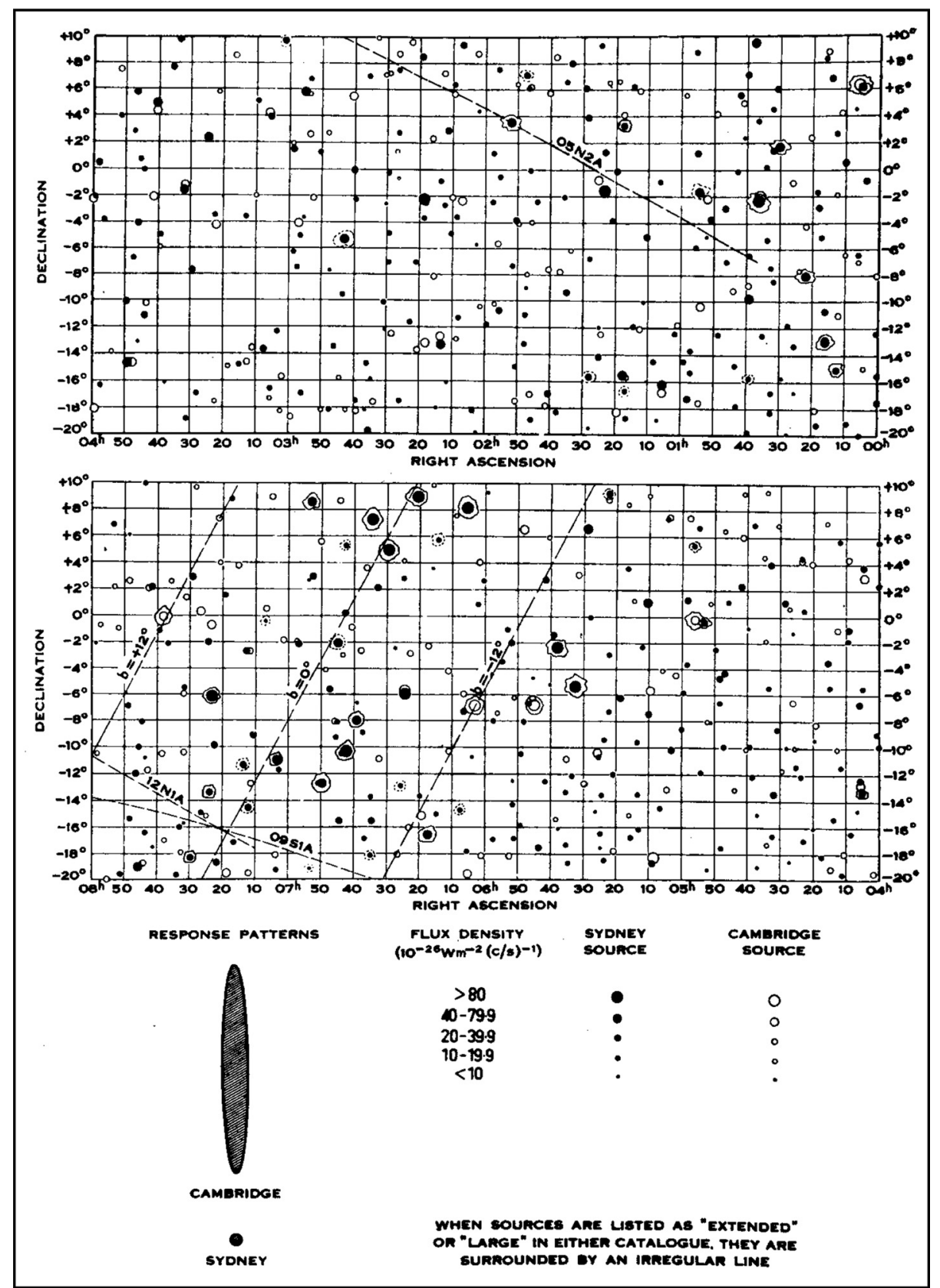

Figure 11 Maps showing sources recorded in the $2 C$ and Fleurs surveys (after Mills and Slee, 1957: 168).

cosmological distance in an evolving Universe ... The steady-state Universe was inadmissible and this, of course, is what worried Hoyle. Although we had just begun observations with the Cross ... it was nevertheless clear from a simple inspection of available records that the slope of the source counts could not possibly be as steep as this. Somewhere the Cambridge group must have gone wrong."

Comparison of source plots in identical regions of the sky showed that the two catalogues were almost completely discordant, and this is well illustrated by reference to Figure 11. Mills and Slee (1957: 167) were convinced that " ... instrumental effects play a decisive part in determining the positions and intensities of sources in at least one of the surveys." and they were equally convinced that the problem lay in England and not in the Antipodes. History was to prove them right, and create a deep rift between the two groups of astronomers, which would take considerable time to heal.

The Mills Cross survey of 1954-1957 produced more than 2000 radio sources, and these were reported in three papers (Mills, Slee and Hill, 1958; Mills, Slee and Hill, 1960; Mills, Slee and Hill, 1961). In their analysis of source counts, Mills et al. distinguished between those of Class I, of low Galactic latitude, and Class II, where $|b|>12.5^{\circ}$. Upon combining data from their 1958 and 1960 papers, they derived a source slope of 1.5 for Class 


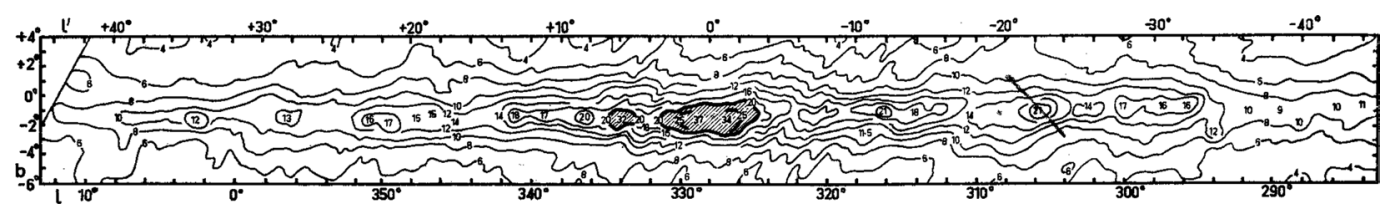

Figure 12 Mills Cross isophote map of the Galactic plane (after Mills, Hill and Slee, 1958: 117).

II sources (Mills, Slee and Hill, 1960), and this result was not altered when sources from their third paper were considered. They concluded that

"...the statistics of the source counts, with due allowance for instrumental effects, show no significant departures from those expected with a random distribution of sources in a static Euclidean universe. They are also not inconsistent with the counts expected in a relativistic expanding universe. The sensitivity of the instrument is inadequate for a definitive result in cosmology based on source counts alone: it seems likely that this method will require numbers of well-determined radio sources approaching 10000 per steradian, at least, to succeed." (Mills, Slee and Hill, 1961: 505-506; my italics).

Source counts alone, whether from Cambridge or Fleurs, could not resolve the cosmological riddle.

Another important outcome from the Mills Cross source survey was the successful search for optical correlates. To Mills and Slee (1957: 174) this was of value in two different ways: as a means of acquiring information about the radio and optical objects, and as a check on the reliability of the Fleurs survey. Several source identifications with spiral, elliptical and irregular galaxies were made, and on this basis Mills, Slee and Hill (1958: 380) concluded that “... a substantial proportion of galaxies may have slightly abnormal radio emission." They also reported several possible associations between Fleurs sources and clusters of galaxies, and foreshadowed the need for further investigations (Mills, Slee and Hill, 1961). This would later prove to be a lucrative research area for Slee. Meanwhile, Mills, Slee and Hill found that several sources correlated with the positions of emission nebulae in the plane of the Galaxy (c.f. Mills, Little and Sheridan, 1956) and three sources were possibly associated with globular clusters, but their attempts to correlate Fleurs sources with planetary nebulae and novae proved unsuccessful. In reality, some years would elapse before improvements in sensitivity would allow the detection of these types of objects at metre wavelengths.

One final result to come out of the Mills Cross survey was a detailed map of radio emission along the Galactic plane between $l=223^{\circ}$ and $13^{\circ}$ and $b=+4^{\circ}$ and $-6^{\circ}$ (Fig. 12). Although earlier surveys of this region had been carried out, including the 'hole-in-the-ground' study at Dover Heights with which Slee was actively involved (see Section 2.10), Hill, Slee and Mills (1958: 530) remind us that until 1954 “... there were no aerials operating at metre wavelengths capable of matching the angular resolution obtainable at centimetre wavelengths."

Towards the end of the Mills Cross survey there was considerable upheaval at Radiophysics as different senior staff lobbied for new radio telescopes, and Mills was one of those who suffered when the Parkes Radio Telescope and Culgoora Radioheliograph were given funding priority (see Robertson, 1992). As a result, in 1960 he accepted a Chair at the University of Sydney, and went on to construct a new and considerably larger 'Mills Cross' at Molonglo, near Canberra.

This, however, was not quite the death-knell of the Fleurs Mills Cross, for during 1961-1962 Slee and Cambridge visitor Peter Scheuer used the east-west arm in conjunction with portable arrays set up at four temporary sites near Sydney in order to investigate the angular sizes of more than $1000 \mathrm{MSH}$ sources (Scheuer, Slee and Fryar, 1963). This project, inspired in part by an earlier interferometric study conducted by Goddard, Watkinson and Mills (1960), promised exciting results. Although the majority of sources with $S \geq 15 \mathrm{Jy}$ at $85.5 \mathrm{MHz}$ were resolved, no quantitative results were published as there was some question about the reliability of the calibrations and the phasing of the antennas. This was a frustrating outcome for all concerned.

\subsection{Scattering and the Solar Corona}

From the very end of WWII solar radio astronomy was one of the research mainstays of the Division of Radiophysics (see Christiansen, 1984). Initially it was pursued at the Dover Heights, Georges Heights and Penrith field stations, but during the 1950s the focus shifted to Dapto, Potts Hill and eventually to Fleurs (for localities see Fig. 4). For a short time in 1947, while still a newcomer to the Division, Slee assisted in the Georges Heights research, but his return to serious solar astronomy only occurred in 1956 once he was ensconced at the Fleurs field station even though he was not a member of the Division's Solar Group.

While the Solar Group's main interests were in solar radio bursts and in radio emission from the quiet Sun, Slee's focus was in the outer solar corona, which he investigated by observing occultations of selected radio sources. The first to employ this technique were Machin and Smith in 1952 using Taurus-A, and they found the substantial reduction in source intensity was best explained in terms of scattering by electron density irregularities high in the solar corona. Hewish carried out similar observations in 1953 and suggested the irregularities were 
probably associated with coronal streamers. Both British teams used interferometers for their observations.

Slee (1956: 228) was aware of certain limitations that interferometers imposed upon such observations, and decided to repeat the British measurements using the eastwest arm of the Mills Cross. Between 1956 May 21 and July 15 he observed Taurus-A as it approached and then receded from the Sun (see Fig. 13). On June 10 the source

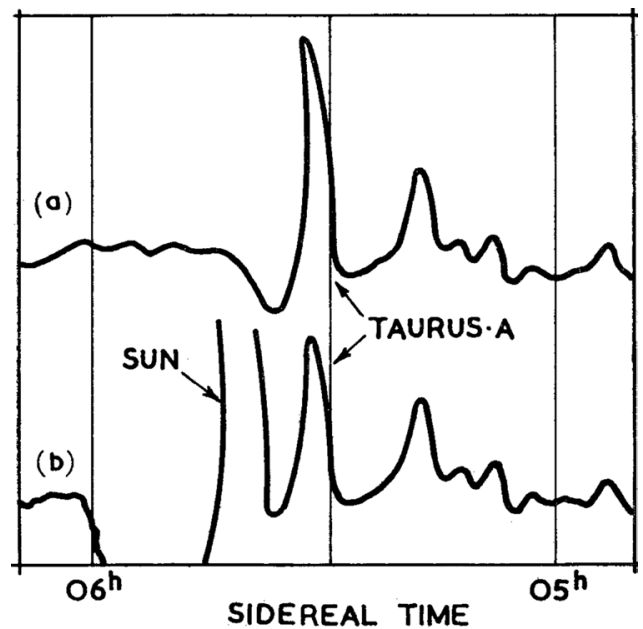

Figure 13 Records of Taurus-A on 1956 June 30 (a), and on 1956 June 16 (b) when the angular separation from the Sun was 11.2 solar radii (after Slee, 1956: 229). Note the considerably reduced source signal in (b). was clearly visible; no observations were made on June 11-13; and on June 14 and 15, when the angular separation from the Sun reached its smallest value, 5.2 solar radii, Taurus-A could not be resolved from the Sun. Slee interpreted the observations as evidence of partial wide-angle scattering by electron density irregularities in the solar corona at angular distances of between 8 and 12 solar radii, but stressed the preliminary nature of these conclusions and pointed to the need for more detailed investigations.

Opportunities to conduct such research occurred in 1957 and 1958 when the Crab Nebula was occulted by the solar corona. In 1957 June, Slee carried out fan-beam, pencil-beam and interferometer observations at $85.5 \mathrm{MHz}$, while in 1958 June only fan-beam and pencil-beam observations were made. The Mills Cross was used for the fan-beam and pencil-beam observations, while the interferometer observations were made with the north-south

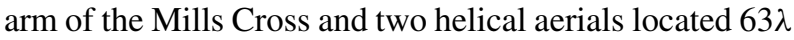
due east of the Cross (see Fig. 14).

In reviewing the observations Slee (1959: 151) concluded that "... the distribution of Crab nebula radiation is markedly affected by refraction and large-scale coronal irregularities. The secondary peak ... was recorded in both 1957 and 1958, and suggests the existence of semipermanent regions in the corona of higher than average electron density." Slee also found evidence of short-term changes in the transmission properties of the corona that were possibly linked to the ejection of disturbances from active

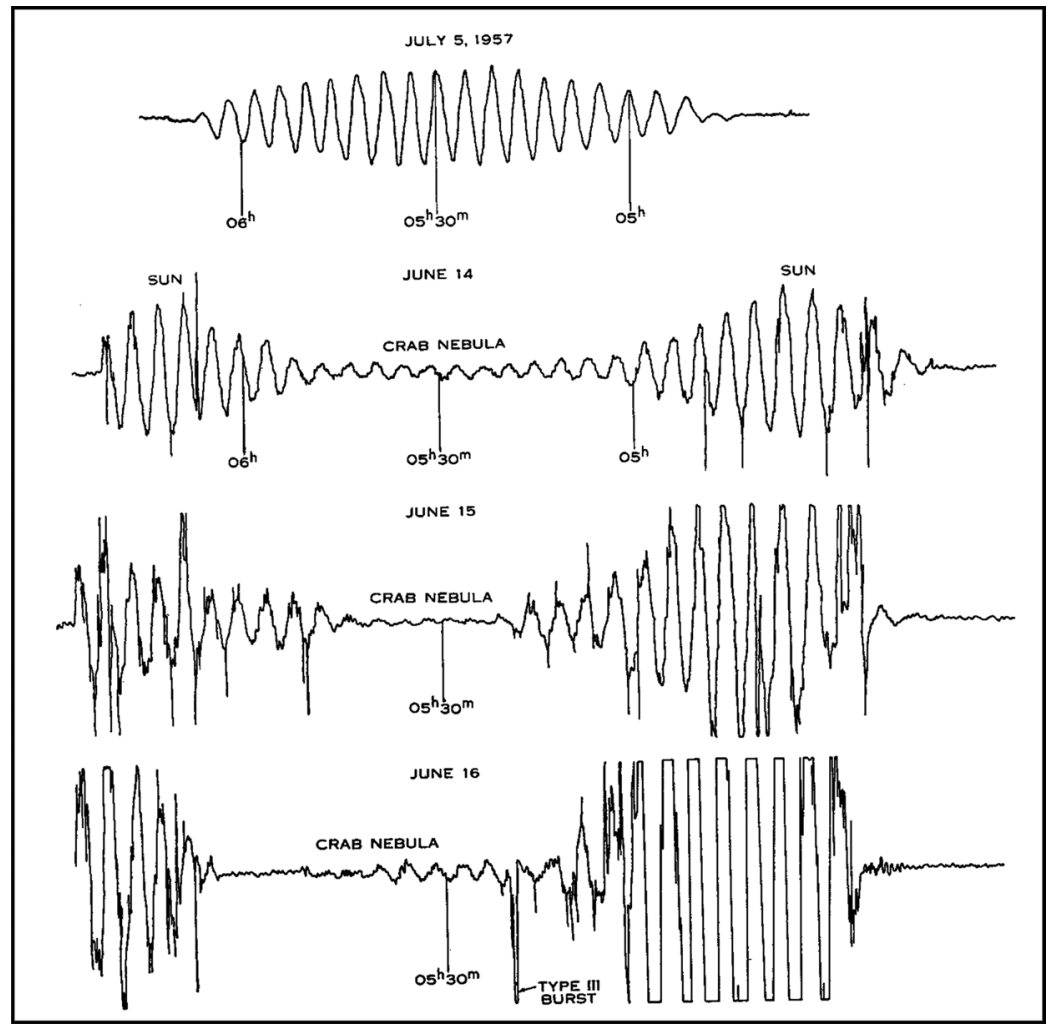

Figure 14 Examples of the interference fringes obtained for Taurus-A on four different dates in 1957. The top trace shows the typical interference pattern when Taurus was well away from the Sun. In contrast, on June 15 the source was occulted by the solar corona, Sun, and the fringes all but disappeared (after Slee, 1959: 141). 
regions on the Sun's disk. In the light of these more complex findings, Slee (ibid.) admitted that the conclusions drawn in his 1956 paper were simplistic.

In a bid to examine the nature of the corona out to 100 solar radii Slee carried out an interferometric study during 1960 June-October using the east-west arm of the Mills Cross at $85.5 \mathrm{MHz}$ and a small array of dipoles $10 \mathrm{~km}$ to the west. With a baseline length of $2920 \lambda$, the primary directivity of the system was $0.8^{\prime}$ east-west and $3.7^{\circ}$ north-south to half-power points. In all, 13 discrete sources with low ecliptic latitudes were examined as they passed closest to the Sun, with the aim of obtaining an indication of the spatial extent of coronal scattering. The results were promising: "Sporadic large increases in the scattering first became noticeable when the angular separation was as much as $100 R_{\odot}$ and at separations of less than $60 R_{\odot}$ the effects of scattering could be detected on every record." (Slee, 1961: 225). Figure 15 provides a graphical illustration of this result, with all 13 sources represented; open circles indicate days when the source interference fringes appeared to be unaffected by scattering, while filled circles refer to days when fringe amplitudes were greatly reduced. The dashed line indicates the region with the majority of filled circles, where the average scattered distribution exceeded 30 arc seconds to half-power points in the east-west direction. Note that at this time the scattering corona was elliptical, with the greatest extension in the equatorial direction, and that
Slee associated this region with an active belt on the visible Sun. Figure 15 reveals another interesting feature: that on several occasions sporadic increases in scattering occurred at distances of between $60 R_{\odot}$ and $120 R_{\odot}$, but almost entirely in the eastern equatorial direction.

During his two-year sojourn at the Royal Radar Establishment (RRE) in Great Malvern, Slee (1966) wrote one last paper on coronal scattering, drawing on observations made at Sydney in 1962 to investigate the way in which the declining solar cycle impacted upon coronal scattering in the $20-80 R_{\odot}$ region. The 1962 interferometric observations were again made at $85.5 \mathrm{MHz}$, but this time using the east-west arm of the Mills Cross in conjunction with a temporary array at a remote site located at a distance of $9200 \lambda$. As a result of observing the sources $3 C 273$ and 3C283, Slee (1966: 261) was able to show that "... as sunspot minimum approached, the scattering irregularities and hence the magnetic fields in the region of interplanetary space $20<R / R_{\odot}<80$ tend to become randomly orientated, no longer preferring the radial direction as appeared to be the case nearer sunspot maximum." In spite of this, Slee found no significant change in the shape of the scattering corona between 1960 and 1962.

In an interesting investigation aimed at looking beyond the immediate outer corona of the Sun, Slee and Higgins explored the nature of the irregularities in the IPM at solar distances of between 1 a.u. and 4 a.u. by measuring scattering suffered by $19.7 \mathrm{MHz}$ Jovian decametric

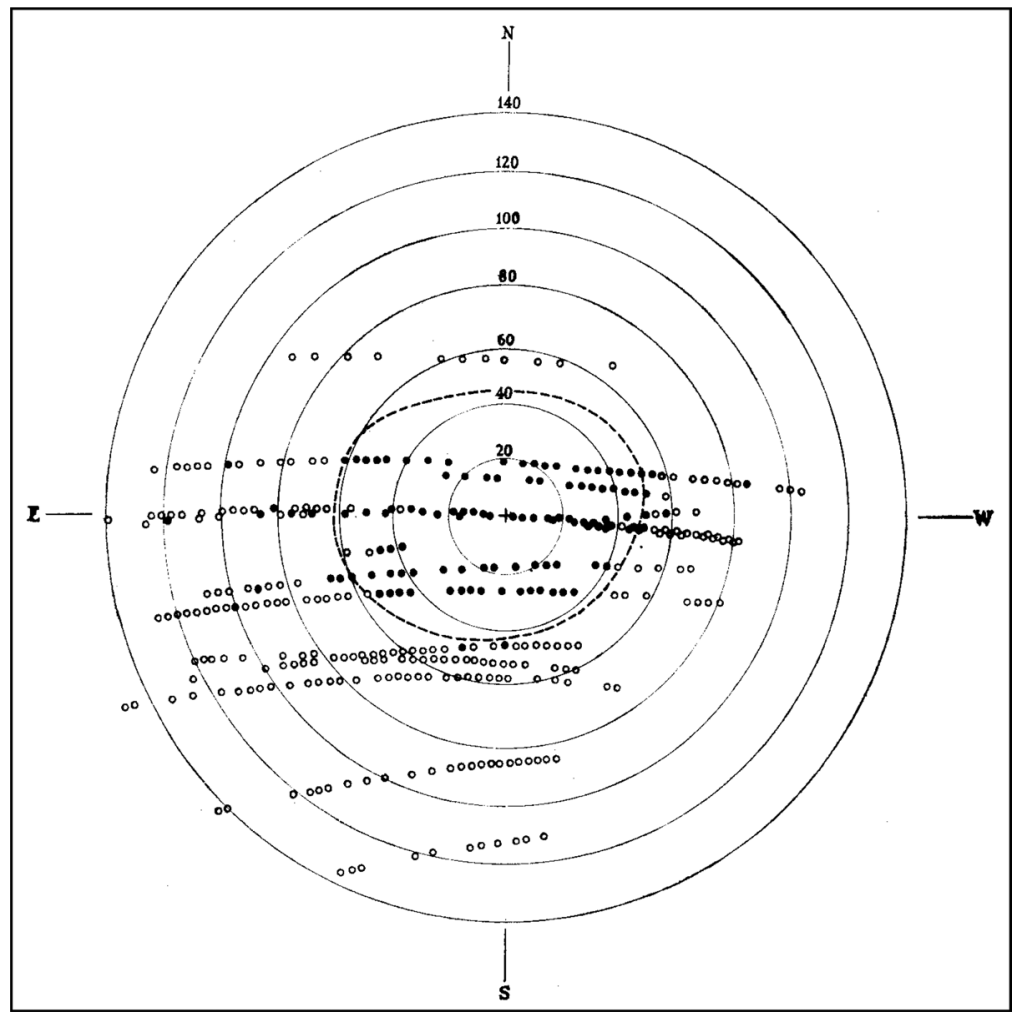

Figure 15 Coronal diagram showing evidence of scattering (filled circles) suffered by 13 discrete sources observed in 1960 (after Slee, 1961: 227). The Sun is marked by the cross, and the concentric circles are spaced at $20 R_{\odot}$ intervals. Coronal scattering was primarily restricted to the region enclosed by the dashed line. 
burst emission received at four different sites in New South Wales, with baselines of $17.4 \lambda, 85.5 \lambda$ and $200.0 \lambda$. Their observations produced the following base data on the IPM and scattering irregularities:

"(1) The solar wind is radially directed with respect to the Sun and possesses an average velocity $V_{\mathrm{s}} \sim$ $600 \mathrm{~km} \mathrm{sec}^{-1}$. (2) R.m.s. deviations in the magnitude and direction of $V_{\mathrm{s}}$, averaged over considerable path lengths through interplanetary space, are less than $20 \%$ and $5^{\circ}$ respectively. (3) The scale size of the irregularities in electron density is approximately $150 \mathrm{~km}$.

(4) The scattering is strong at $19.7 \mathrm{MHz}$ for all directions in the ecliptic plane; the r.m.s. phase modulation varies from about $1 \mathrm{rad}$ near opposition to about $11 \mathrm{rad}$ at an elongation of $20^{\circ}$. (5) The conclusions in (3) and (4) are consistent with an r.m.s. electron density that varies with heliocentric distance ... (6) The irregularities in electron density with dimensions of hundreds of kilometres are relatively weak $(10 \%)$ in comparison with the average electron density." (Slee and Higgins, 1968: 342).

Finally, it should be mentioned that in order to investigate electron density variations in the IMP Cole and Slee subsequently used a new type of radio spectrograph capable of recording source scintillations over a two-to-one frequency range. On 1978 October 10 and 11 the source 3C273 was observed with the Parkes Radio Telescope and an acousto-optical radio spectrograph that scanned the spectral range $280-520 \mathrm{MHz}$, and Cole and Slee (1980) published their interpretation in Nature. They believed that the radio spectrograms could not be explained in terms of conventional models of the IPM, but in an accompanying note Hewish (1980) claimed to the contrary, suggesting instead that Cole and Slee's “... beautiful observations of interplanetary scintillation ... reinforce the standard theory." Although this radio spectrograph had the potential to provide valuable data on source scintillations, Slee decided not to take up the challenge, for by this time he had other research priorities and commitments.

\subsection{The Source of the Jovian Burst Emission}

The serendipitous discovery of Jovian emission by Burke and Franklin (see Nature, 1955) opened the whole new theatre of planetary radio astronomy, and by the time Slee became involved in this area of research an association with the Jovian System III longitude was already well established (see Smith and Carr, 1964). What was not understood was the identity and nature of the emitting source (or sources), and during the 1962 opposition Slee and Higgins employed long baseline interferometry in order to investigate this. For this project, they used square arrays of 19.7 MHz half-wave dipoles, one located at Fleurs and the other at a remote site distant $32.3 \mathrm{~km}$ to the south. With an interferometer spacing of $1940 \lambda$, the observations showed that the "... angular diameter of a burst source was ... less than a third of the planet's diameter, and that all bursts contributing to a noise storm

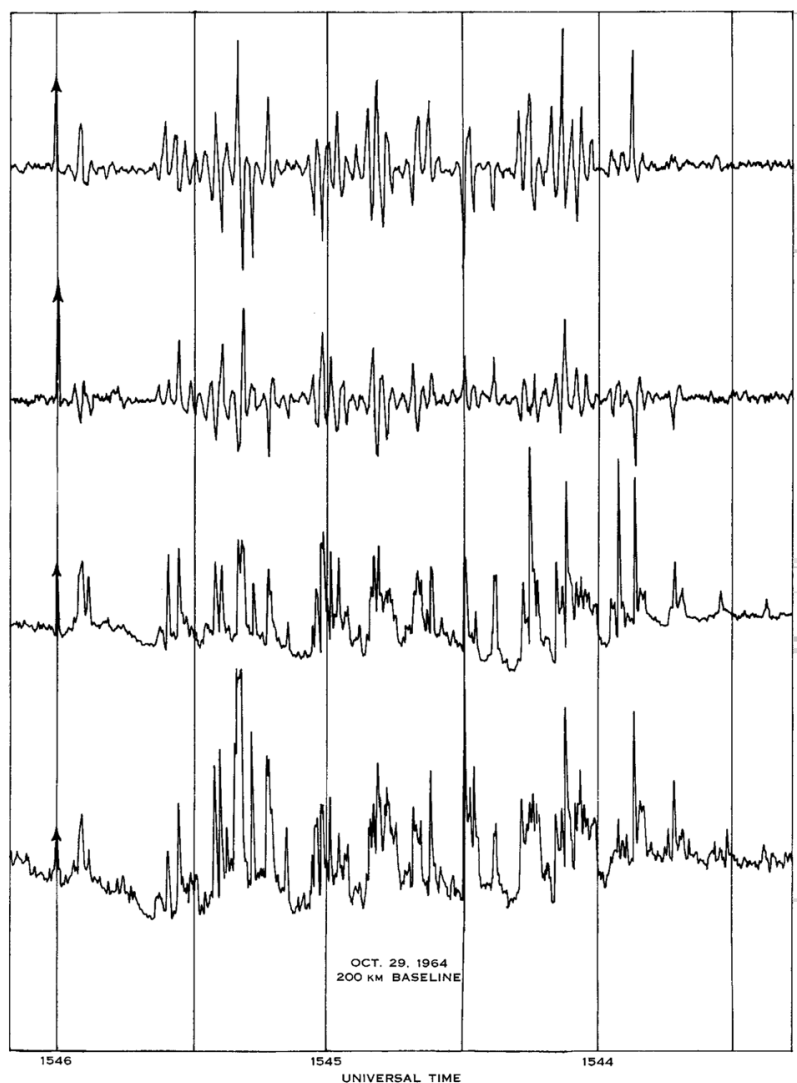

Figure 16 Examples of 19.7-MHz chart records from Jamberoo and Heaton. The two upper traces show the sine and cosine components of the interferometer patterns, and the two lower traces are total power records from the two sites (after Slee and Higgins, 1966: 171).

originated in a single area less than a half of the size of the planet's disk." (Slee and Higgins, 1963: 781-782).

On the basis of this initial result Slee and Higgins decided to continue these investigations using longer baselines, and in 1963 and 1964 they employed effective spacings of between $691 \lambda$ and $12700 \lambda$. Simple arrays of dipoles at Fleurs and three other sites, Dapto, Jamberoo and Heaton (see Figs 2 and 4 for these localities), were phased and oriented to receive $19.7 \mathrm{MHz}$ Jovian emission over an hour-angle range of $\pm 4 \mathrm{~h}$ (see Fig. 16), and a radio link was used to transmit the signals from the remote sites to Fleurs.

Analysis of the observations revealed that

“... apparent source sizes were usually in the 10-15 sec of arc ... [but] the source sizes measured may not be intrinsic, but may be yet another manifestation of the diffraction or focussing process ... Such an interpretation would allow the irregularities responsible for the scintillations to be placed at greater distances from the Earth, where effective focusing could take place. However, the angular size condition would now dictate a small intrinsic angular size for the Jupiter source, probably less than $1 \mathrm{sec}$ of arc to half-brightness points... . If, as seems possible, scattering is a major contributor to the apparent source size, then it is unlikely that the 


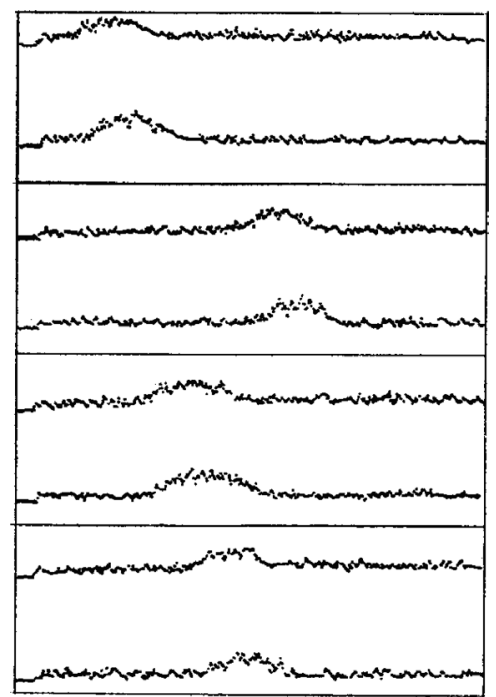

(a)

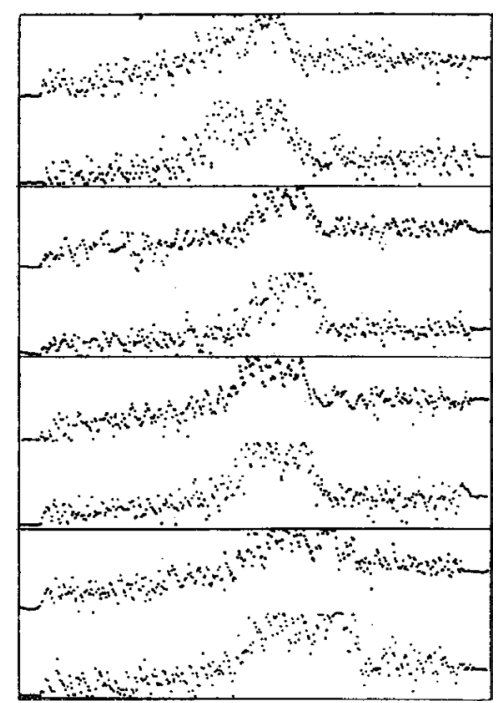

(b)

Figure 17 Examples of $20 \mathrm{MHz}$ bursts obtained on 1966 January 23 (a) and 1965 December 23 (b). The plots in (a) show four successive bursts recorded at the two ends of the baseline, while (b) shows four other bursts with millisecond structure (after Slee and Gent, 1967: 236).

intrinsic source size will be measured by interferometric methods." (Slee and Higgins, 1966: 179-180).

This result turned the Radiophysics Jovian decametric project in a new direction: what had started as a quest for emission source size now became an investigation of scattering by the interplanetary medium. Slee and Higgins (1968) then used 1963-1964 data on burst arrival times, burst rates, angular position scintillations and apparent angular size to successfully investigate interplanetary diffraction patterns and electron irregularities in the solar wind.

Slee's final foray into Jovian decametric emission occurred in 1965-1966 while he was based at the RRE. One of his projects during this English sojourn was an investigation of the differential arrival times of $20 \mathrm{MHz}$ Jovian bursts at three spaced antennas. Slee and Gent (1967: 235) were able to demonstrate that millisecond bursts were not imposed by the interplanetary medium, but "... are caused by variation of amplitude or frequency in the source itself." In the course of their investigation they also discovered a new type of sub-millisecond burst (see Fig. 17b), which was seen as a basic component of all intense Jovian decametric emission.

Several years later, after the Culgoora Radioheliograph was operational, Slee turned to Jupiter one last time. However, his interest on this occasion was not in the decametric burst emission, but rather the flux emitted by the planet at $80 \mathrm{MHz}$. Using Radioheliograph data assembled during the first half of 1970, Slee and Dulk (1972) came up with a Jovian flux value of $6.0 \pm 0.7 \mathrm{Jy}$, similar to the figure of $4.5 \pm 1.0 \mathrm{Jy}$ previously reported by Gower. On the basis of their value and assembled decametric measurements they concluded that the Jovian spectrum was substantially flat down to at least $80 \mathrm{MHz}$., a view at variance with that published by Gleeson, Legg and Westfold in 1970.
More recently, Slee and Dulk's interpretation has been confirmed by other researchers (see Artyukh, Kuz'min and Makarov, 1972; de Pater and Butler, n.d.).

As a matter of interest, the only other planet that Slee tried to observe was Uranus. On 1964 February 24, May 2 and May 3 he carried out an unsuccessful search for radio emission at $2655 \mathrm{MHz}$ using the Parkes Radio Telescope, and was able to set an upper limit of $1.77 \mathrm{Jy}$ for Uranian emission on these dates, corresponding to an equivalent temperature of $300 \mathrm{~K}$ (Slee 1964). Later this same year, Kellermann (1966) also observed Uranus with the Parkes Radio Telescope at $2655 \mathrm{MHz}$. By employing the on-off observing mode and a substantially longer integration time he was able to obtain an equivalent blackbody temperature of $130 \pm 40 \mathrm{~K}$.

\subsection{The Plasma Tails of Comets}

In 1957 April, Shain and Slee (1957) tried to observe C/1956 R1 (Arend-Roland) at $19.7 \mathrm{MHz}$ and $85.5 \mathrm{MHz}$, using the Shain Cross and Mills Cross respectively, but like Whitfield and Hogböm (1957) they were unsuccessful. History has shown that another 16 years would pass before radio telescopes with the requisite sensitivity would detect emission from comets (see Biraud et al. 1974; Turner, 1974).

Fully 30 years after his initial assault on a comet Slee returned to cometary astronomy, but he was joined on this occasion by Australian and Indian colleagues. Moreover, his interest was not in the radiation emitted by these bodies but rather in the features of their plasma tails, and in order to investigate these parameters he returned to a familiar phenomenon: source scintillations.

Comet 1P/Halley offered the first opportunity for Slee to apply this technique to a comet, and on 1986 March 29 he observed the strong compact radio source 1827-360 at 


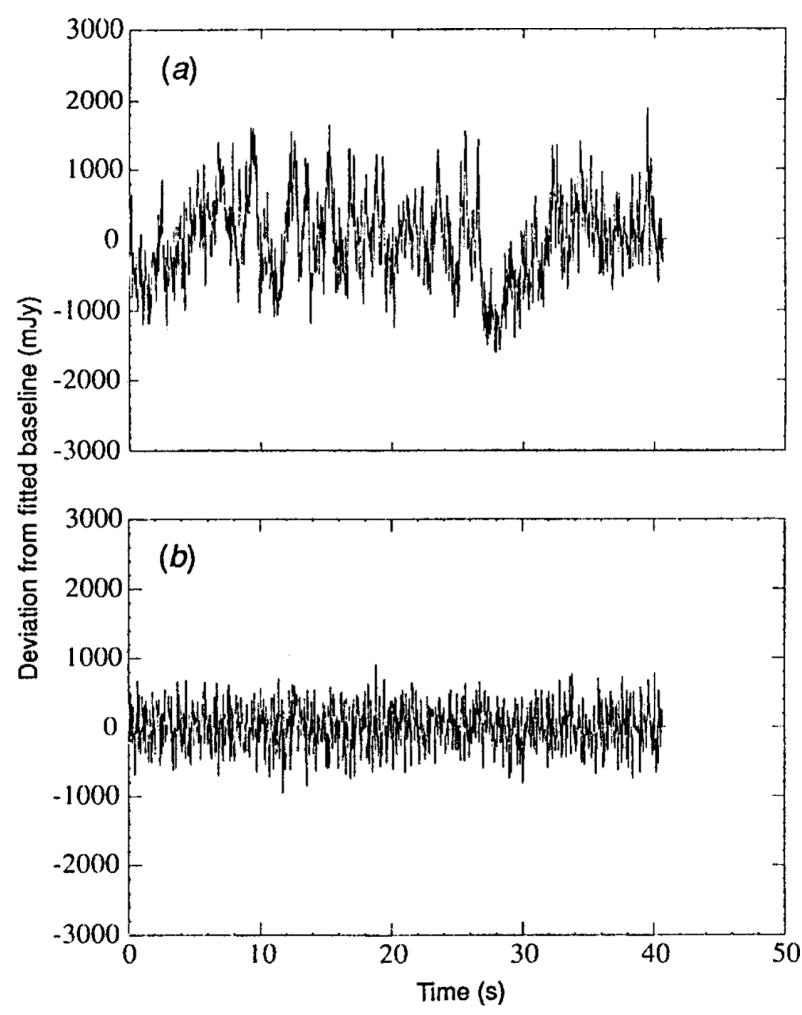

Figure 18 Plots of the signal from 0637-752 when the pulsar was behind the tail of Comet C/1986 P1-A (Wilson) (a) and at a later date (b), when the pulsar was well away from the comet (after Slee, Bobra, Waldron and Lim, 1990: 805).

$408 \mathrm{MHz}$ using the Parkes Radio Telescope as the plasma tail of the Comet swept across the source. Enhanced source scintillations were observed, and these were interpreted as the result of diffraction of radio waves by electrondensity irregularities in the plasma tail. Furthermore, "At a distance of $5.4 \times 10^{6} \mathrm{~km}$ downstream from the cometary nucleus the central part of the plasma tail contained electron density irregularities about 14 times as strong as those in the undisturbed solar wind." (Slee, McConnell, Lim and Bobra, 1987: 699). Despite these findings, and the publication of similar results by Ananthakrishnan et al., and Alurkar et al., others were unable to detect enhanced intensity scintillations and there was controversy over their very existence.

The chance to resolve this impassé came on 1987 May 1 and 2 when the plasma tail of C/1986 P1-A (Wilson) swept across two quasars, 0606-795 and 0637-752 (Fig. 18). On this occasion, Slee and his collaborators carried out observations with the Parkes Radio Telescope at $408 \mathrm{MHz}$, and two different types of scintillations were clearly detected: a small-scale turbulence of $10-40 \mathrm{~km}$ that developed near the tail-axis, and large-scale turbulence of $90-350 \mathrm{~km}$ that was present in the transition region between the plasma tail and the solar wind (Slee, Bobra, Waldron and Lim, 1990). There could now be no doubt that comet tails caused enhanced intensity scintillations.

Three years later, C/1989 X1 (Austin) provided Slee and his colleagues with a third opportunity to investigate the plasma turbulence found in and near a cometary tail.

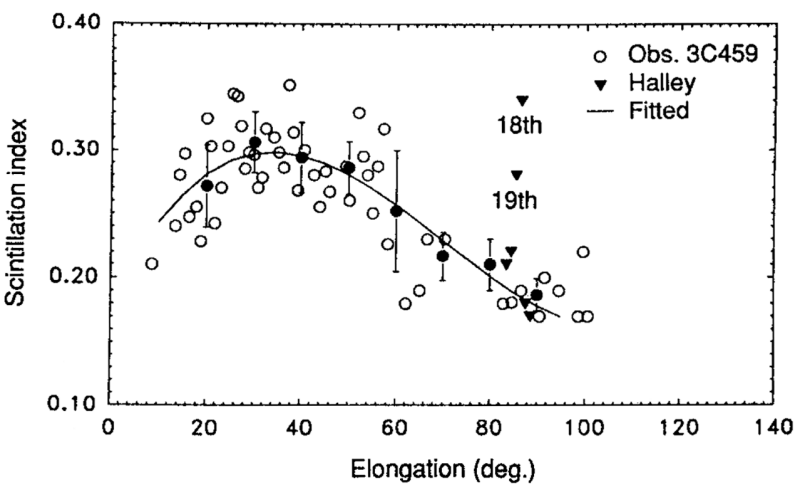

Figure 19 Plot of the variation in scintillation index with solar elongation for the quasar $2314+038$. The filled circles represent binned mean values (after Janardhan, Alurkar, Bobra, Slee and Waldron, 1992: 117).

This time the observations were carried out in India, using the $20000 \mathrm{~m}^{2}$ dipole array operating at $103 \mathrm{MHz}$ maintained by the Ahmedabad Physical Laboratory. The target date was 1990 May 13 when the Comet's tail passed in front of the quasar 2204+29 (3C441), and once again enhanced intensity scintillations were observed and the small-scale turbulence first noted for Comet Wilson was again detected. Janardhan, Alurkar, Bobra and Slee (1991: 565) found that "The r.m.s. electron density variation $\Delta N$, at a distance of 0.9 A.U. from the sun and $7.3^{\circ}$ downstream from the nucleus, was found to be $6 \mathrm{~cm}^{-3}$ as compared with $1 \mathrm{~cm}^{-3}$ for the normal solar wind." They also found that the scale sizes of the turbulence were much finer than normally found in interplanetary scintillations caused by the solar wind.

After these findings for Comets Wilson and Austin, Janardhan, Alurkar, Bobra, Slee and Waldron (1992) carried out an analysis of the quasar 2314+038 (3C459) that was occulted by the plasma tail of Comet $1 \mathrm{P} /$ Halley in 1985 December. Once again enhanced scintillations were present (see Fig. 19), and they found evidence of fine-scale turbulence of $9-27 \mathrm{~km}$ near the tail-axis and large-scale turbulence of $100-265 \mathrm{~km}$ in the transition region between the plasma tail and the solar wind.

\subsection{Radio Flares from dMe Stars}

By 1960 the non-stellar identity of the so-called 'radio stars' was beyond doubt, and improvements in sensitivity made it possible to search for genuine radio stars. The first targets were the dMe flare stars, and the reasons were obvious:

"Detection of radio emission from flare stars might lead to several important new fields of research. For example, although probably 80 percent of all main sequence stars are $\mathbf{M}$ dwarfs, we know little about the physical conditions in their atmospheres. Studies of radio bursts - which could be generated in these stars' coronas, if indeed they have extensive atmospheres like the sun - would be valuable in this connection."

"Again, statistics of flare-star bursts may tell whether stars in general possess 'star spot' cycles analogous to 


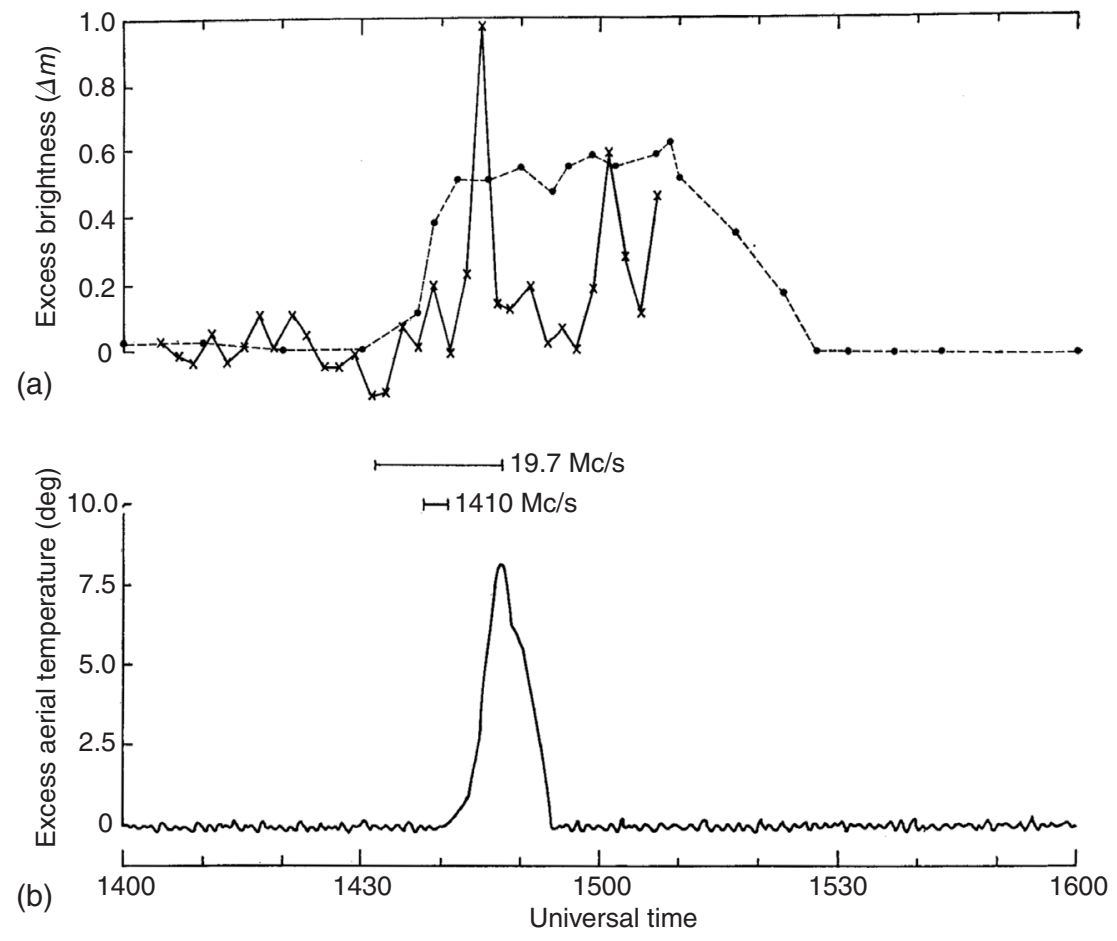

Figure 20 Optical and radio flare on V371 Orionis recorded on 1962 November 30. The optical light curves are plotted in (a), with the crosses showing the photographic curve from the Baker-Nunn camera at Woomera and the dots a group of Sydney amateur astronomers. The smoothed $410 \mathrm{MHz}$ radio burst is shown in (b) together with an indication of the time intervals over which 19.7 and $1410 \mathrm{MHz}$ emission was recorded (after Slee, Solomon and Patston, 1963: 993).

the 11-year sun-spot cycle. Finally, radio studies could show whether flare stars eject high-energy particles in sufficient amount to contribute appreciably to cosmic rays and, indirectly, to the radio emission of the Milky Way galaxy." (Slee, Higgins and Patston, 1963: 83)

From 1960 September until 1962 May, Slee and Higgins carried out intermittent monitoring of four different flare stars: UV Ceti (1960 and 1961), Proxima Centauri (1960 and 1962), V371 Orionis (1961 and 1962) and V1216 Sagittarii (1962). Observations were mainly achieved with the north-south arm of the $85.5 \mathrm{MHz}$ Mills Cross at Fleurs, but the north-south arm of the $19.7 \mathrm{MHz}$ Shain Cross and the Parkes Radio Telescope (at 400 and $1500 \mathrm{MHz}$ ) were used for some observations. During some of the radio monitoring, teams of amateur astronomers from New South Wales, Victoria and Queensland carried out parallel optical observations. Two definite optical flares were recorded but neither was accompanied by $85.5 \mathrm{MHz}$ radio emission, however, within a few minutes of three of the eleven possible optical flares "... radio deflections were recorded consistent with an origin at the flare star. In all three cases ... the 3.5-meter [85.5 MHz] bursts were small, but during the possible flare of UV Ceti on November 13, 1961, concurrent observations at 15 meters [19.7 MHz] revealed an extremely intense group of bursts, about four minutes after a similar but much weaker group on 3.5 meters." (Slee, Higgins and Patston, 1963: 85). Radio bursts were also detected at $85.5 \mathrm{MHz}$ on five occasions when there were no optical observations. In a popular article in Sky and Telescope reporting on this work, Slee, Higgins and Patston included two figures showing possible radio bursts from UV Ceti.

At about the same time, Lovell and his US-based collaborators reported in Nature on their own search for radio emission from flare stars, and Slee felt that these two surveys collectively established that at least some flare stars were active at radio wavelengths (see Slee, Solomon and Patston, 1963: 991).

Later in 1963, Slee's team was able to publish an account of a particularly intense radio flare on V371 Orionis, which accompanied an optical flare recorded visually and photographically in Australia (see Fig. 20). Radio monitoring was carried out at 410 and $1410 \mathrm{MHz}$ with the Parkes Radio Telescope and at $19.7 \mathrm{MHz}$ with the north-south arm of the Shain Cross, and although the radio deflection was very conspicuous at $410 \mathrm{MHz}$ (see figure 1 in the paper by Slee, Solomon and Patston, 1963), emission was also detected at the other two frequencies. Slee et al. noted that there was no significant time difference in the onset of radio emission at the three frequencies - unlike Type II solar bursts. They then proceeded to compute the radio energy liberated by V371 Orionis at the three frequencies during the outburst, and found a solar comparison illuminating: "One of the most important solar radio events was that of February 21/22, 1950 , which yielded about $10^{18}$ joules over the frequencyrange $100-10000 \mathrm{Mc} / \mathrm{s}$, a value which is below the radio energy of the V371 Orionis event by a factor of $10^{6}$." (Slee, Solomon and Patston, 1963: 992). The origin of this stellar radio flare obviously involved a non-thermal mechanism. 
Rather than focus on just the one flare star, Slee then turned his attention to the Orion Nebula, known to harbour numbers of flare and flash stars, and in 1963 December and 1968 November he and three colleagues carried out simultaneous radio and optical observations. The radio monitoring was accomplished with the Parkes Radio Telescope, operating at $136 \mathrm{MHz}(1963), 150 \mathrm{MHz}$ (1968) and $408 \mathrm{MHz}$ (1963 and 1968), while the optical observations were made with the Uppsala Schmidt Telescope at Mount Stromlo Observatory. During $34.1 \mathrm{~h}$ of coordinated observing, nine bright optical flares were detected, all but one of which were accompanied by obvious radio emission at one or more frequencies. Slee, Higgins, Roslund and Lyngå (1969: 1089) found that there were two different types of radio flares: (1) short impulsive events lasting 1.5-5 min, with high peak flux densities, and (2) smooth bursts of lower peak flux density with durations an order of magnitude longer than those described in (1). An example of the second type of radio flare is shown in Figure 21. Slee and Higgins then went on to provide a more detailed account of the radio flares and noted that their energies were many orders of magnitude higher than their solar counterparts. They then discussed possible energy-generating mechanisms, concluding that "The high radio brightness temperatures of $10^{18}-10^{20} \mathrm{~K}$ cannot be due to incoherent synchrotron emission because of limitations due to self-absorption. The coherent mechanisms of plasma oscillations and amplification of gyro or synchrotron radiation can account for the radio observations without invoking the presence of abnormally dense particle streams, strong magnetic fields, or high ambient electron densities." (Slee and Higgins, 1971a: 247).

As a matter of interest, about two decades later Slee joined two US-based collaborators in a search for radio emission from flare stars in the Pleiades. This open cluster contains large numbers of flare stars, and between 1984 January and 1985 March fields containing 40 known flare stars were examined with the VLA at 1.4, 4.9 and $15 \mathrm{GHz}$, but no radio detections were made at $\geq 0.3 \mathrm{mJy}$, despite this cluster being much closer to us than the Orion Nebula (Bastian, Dulk and Slee, 1988).

After their successful observation of radio emission from flare stars in the Orion Nebulae during the 1960s, Slee and his collaborators turned to individual flare stars, and the first to attract their attention — albeit briefly — was AD Leonis. Primary observations at 80 and $160 \mathrm{MHz}$ with the Culgoora Radioheliograph and supplementary data recorded with the Molonglo Cross at $408 \mathrm{MHz}$ and the 64-m Tidbinbilla antenna at $2295 \mathrm{MHz}$ revealed a weak variable radio source 1.85 arc min south-east of $A D$ Leonis, but its variability, spectral index, and polarisation suggested that this was simply a chance juxtaposition and that the source was really a normal radio galaxy or a QSO (Robinson, Slee and Little, 1976). Subsequent research confirmed these suspicions (see Slee and Page, 1979).

Slee and five Australian and New Zealand colleagues then selected 14 well known flare stars and between 1972 January and 1976 September they carried out a cooperative radio and optical observing program. The radio observations were made at 80 and $160 \mathrm{MHz}$ with the Culgoora Radioheliograph, while 17 different amateur and professional observatories were involved in the optical monitoring. A total of 48 radio flares at $80 \mathrm{MHz}$ and 114 flares at $160 \mathrm{MHz}$ was detected, associated with 11 of the 14 stars surveyed. During 110 h of simultaneous observing 21 optical and 19 radio flares were recorded, but only four pairs appeared to be correlated. Analysis of all radio flares showed that they had mean durations of $\sim 1.7 \mathrm{~h}$, were sometimes highly circularly polarised and displayed non-thermal radio spectra (Nelson et al. 1979: 417).

The focus of the Radiophysics flare stars program then shifted to detailed multi-wavelength examinations of individual stars, and the first of these involved coordinated X-ray, optical and radio observations of YZ

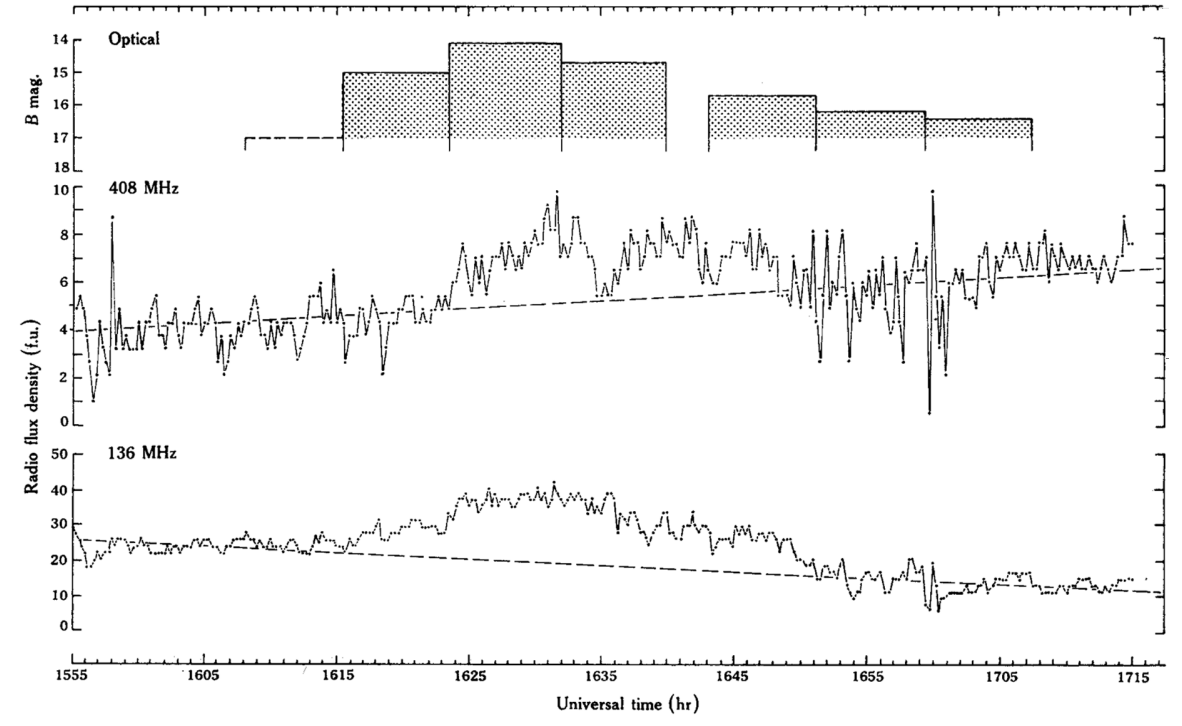

Figure 21 Example of a longer-duration optical and radio flare event, observed at two radio frequencies, and recorded on 1963 December 21 (after Slee, Higgins, Roslund and Lyngå, 1969: 1088). 
Canis Minoris between 1975 November 30 and December 3. This was the first occasion upon which a flare star had been monitored in all three spectral regions simultaneously, with the radio observations contributed by the Culgoora Radioheliograph (160 MHz) and Parkes Radio Telescope $(5000 \mathrm{MHz})$ in Australia plus other instruments in India, Puerto Rico and the USA. In a paper with 27 authors (only three of whom were from the Division of Radiophysics in Australia), Karpen et al. (1977: 479) announced the detection of 31 minor optical flares and 11 radio flares, and stated that "Although no flare-related $\mathrm{X}$-ray emission was observed, the measured upper limits in this band enable meaningful comparisons with published flare-star models. Three of the five models predicting the relative $\mathrm{X}$-ray to optical or radio flare luminosities are in serious disagreement with the observations."

Slee was next involved in an Australian-South AfricanUSA multi-wavelength assault on the nearest and arguably the best known of all flare stars, Proxima Centauri, for three days in 1977 May. In addition to the Parkes Radio Telescope (operating at $5000 \mathrm{MHz}$ ), radio data were also contributed by the $25-\mathrm{m}$ antenna at Hartebeesthoek in South Africa. While 30 optical flares and 12 possible radio bursts were recorded, the $S A S 3 \mathrm{X}$-ray satellite made no $\mathrm{X}$-ray detections. On the basis that no $5000 \mathrm{MHz}$ emission was received by the Parkes antenna during one major and three minor optical flares, Haisch et al. (1978) were able to derive an upper limit for the flare radio emission of $L_{\mathrm{R}} / L_{\text {opt }}<2 \times 10^{-5}$.

Haisch organised another coordinated Proxima Centauri program for 1979 March 6/7, this time involving simultaneous X-ray, ultraviolet, optical and radio observations, and once again Slee was a participant. On this occasion, 5 optical flares and 12 possible radio bursts were recorded, including one flare with simultaneous optical and radio emission. In addition, one major X-ray flare was observed by the HEAO $2 \mathrm{X}$-ray satellite, but there was no corresponding ultraviolet, optical or radio emission. Haisch et al. (1981: 1009) interpreted the absence of emission at these wavelengths "... in terms of an arch model in which the flare cools predominantly by X-ray radiation. The observed $20 \mathrm{~min}$ exponential cooling time is consistent with an electron density of $1.0 \times 10^{11} \mathrm{~cm}^{-3}$ during the decay phase and a flare of total arch length of $\pi \times 10^{10} \mathrm{~cm}$, comparable to the size of the star itself."

The next flare star to attract Slee's attention was AT Mic, and in 1980 October he and three Australian collaborators carried out $5000 \mathrm{MHz}$ radio observations with the Parkes Radio Telescope and photographic monitoring with the $66-\mathrm{cm}$ Yale Columbia refractor at Mount Stromlo Observatory. One major flare was recorded simultaneously at optical and radio wavelengths, which was notable for its long duration and good temporal correlation between optical and microwave intensities. Slee, Tuohy, Nelson and Rennie (1981: 220) concluded that "Unless the dimensions of the source are very much larger than those observed for solar flares, the $5-\mathrm{GHz}$ source has too great a brightness temperature to be produced by the gyrosynchrotron mechanism ... Much higher energy particles or a coherent emission mechanism must be involved in the stellar microwave flare."

After working intermittently on flare star radio emission for more than 20 years, Slee joined with four Australian and New Zealand colleagues in preparing a review of microwave emission from dMe stars, and this was presented at the Second Asian-Pacific Regional Meeting on Astronomy, in Bandung, Indonesia, in 1981 August and subsequently published in the Conference Proceedings (Slee, Allen, Coates, Page and Quinn, 1984).

The success of the AT Mic collaboration of 1980 led to a more ambitious optical-radio project in 1982 and 1983, also involving colleagues from Macquarie University in Sydney and Perth Observatory. In 1982 July, optical observations were carried out photometrically with the 3.9-m Anglo-Australian Telescope (AAT) simultaneously in $K$ band and in $U$ or $B$ band, while in 1983 August coordinated photometric observations were made at Mount Stromlo with the 1.9-m telescope in $J$ band and the $75 \mathrm{~cm}$ telescope in $U$ band, and at Perth Observatory with the $60-\mathrm{cm}$ telescope in $B$ band. During both periods, radio observations were made with the Parkes Radio Telescope at $5000 \mathrm{MHz}$ and the Molonglo Synthesis Telescope (MOST) at $843 \mathrm{MHz}$. The observations produced 32 optical flares, five of which were accompanied by definite or possible radio emission at $5000 \mathrm{MHz}$ (e.g. see Fig. 22). In addition, six radio bursts were recorded at Parkes; for two of these there were no optical counterparts, while clouds

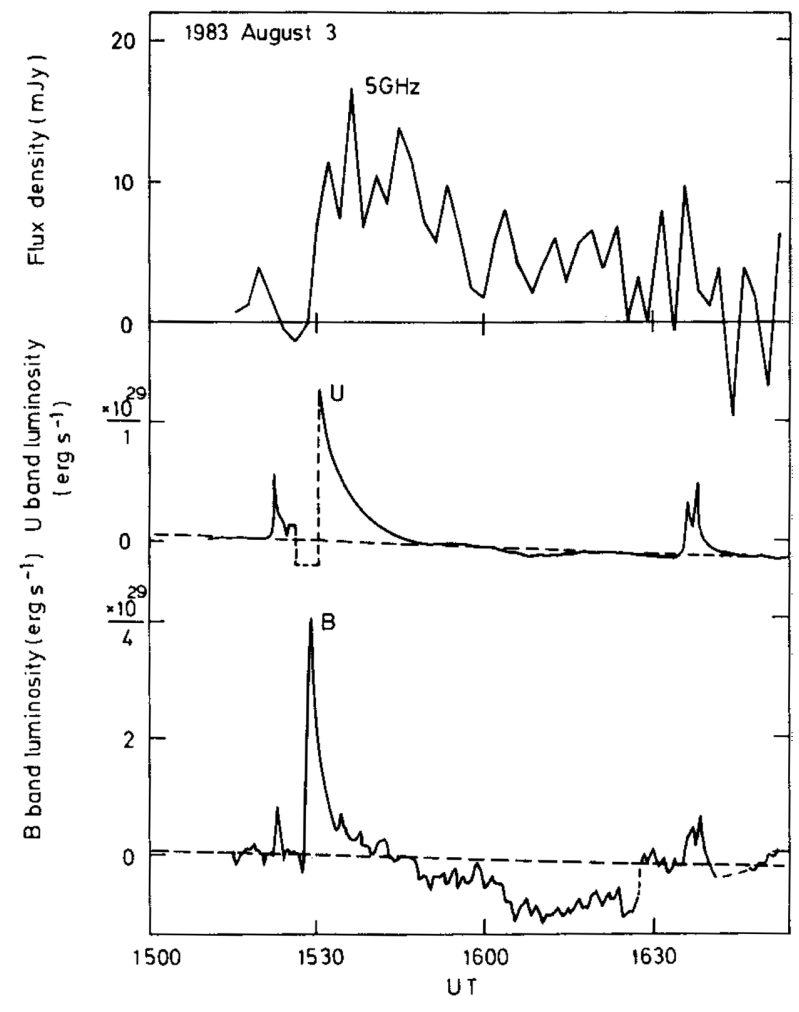

Figure 22 Example of a short-duration optical and radio flare event on AT Mic, observed on 1983 August 3 (after Nelson et al. 1986: 100). 
prevented optical coverage of the remainder. In contrast, no radio flares were detected with the MOST, placing an upper limit of 100-200 mJy on flare emission and 3 mJy on the quiescent emission. Interesting, none of the optical flares showed a decrease in infrared flux, as expected if the inverse Compton scattering mechanism invoked by Gurzadyan were to explain the stellar flare phenomenon. To the contrary, one optical flare was accompanied by a significant increase in $K$ band flux. Nelson et al. (1986: 91) believed these results supported a hot $\left(10^{5} \mathrm{~K}\right)$ plasma flare model which produced a relatively flat energy-spectrum over a wavelength range determined by the temperature, density and depth of the source region.

In 1996, Slee and Taiwan- and US-based colleagues addressed a familiar target, Proxima Centauri. On this occasion they dealt only with radio observations, which were made in 1990 July (5000 MHz), 1991 August (1500, 2300, 5000 and $8570 \mathrm{MHz}$ ), 1993 September (5000 and $8750 \mathrm{MHz}$ ) and $1994 \mathrm{May}(5000$ and $8750 \mathrm{MHz})$. During a total of $85 \mathrm{~h}$ of radio monitoring only one radio flare was detected. On the basis of the radio observations at 5000 and $8750 \mathrm{MHz}$, Lim, White and Slee (1996a: 976) placed an upper limit of $\sim 2 \times 10^{11} \mathrm{ergs}_{\mathrm{Hz}^{-1}} \mathrm{~s}^{-1}$ on its radio luminosity, which is ".. the lowest detection threshold yet reached for a star other than the Sun. This upper limit is approximately equal to the radio luminosity of the active Sun ... outside of flares." The authors also examined the filling factor of 500-1000 G magnetic loops containing $\mathrm{X}$-ray emitting plasma on Proxima Centauri, and placed an upper limit on the mass-loss rate by any stellar wind from the star.

Slee's most recent paper on flare stars (Robinson, Airapetian, Slee, Mathioudakis and Carpenter, 2001: 21) discusses multiwavelength observations of $\mathrm{YZ} \mathrm{CMi.}$ Research on flare stars is on-going and further papers are anticipated.

\subsection{Radio Emission from Other Active Stars?}

Buoyed on by his initial success with flare stars Slee then turned to the quest for radio emission from other active stars (pulsars excepted!), in part inspired by the research program of Andew Cameron Collier. At this time, Collier was completing a Ph.D. at the University of Canterbury (Christchurch, NZ) on spectroscopic properties of southern RS CVn stars, and he encouraged Slee to search for radio emission from these chromospherically active binaries.

Collier, Slee and three other Australian astronomers began by collaborating on a coordinated radio and $\mathrm{H} \alpha$ survey of southern RS CVn systems and related stars in 1981 July. The radio observations were made at $5000 \mathrm{MHz}$ with the Parkes Radio Telescope, and the $\mathrm{H} \alpha$ survey with the 1.88-m reflector at Mount Stromlo Observatory. While 21 stars were on the observing list, three of these were not strictly members of the RS CVn class. Two were single stars with strong $\mathrm{H} \alpha$ and $\mathrm{Ca}$ II emission, both members of the FK Comae class, while the third, HD 101379, was
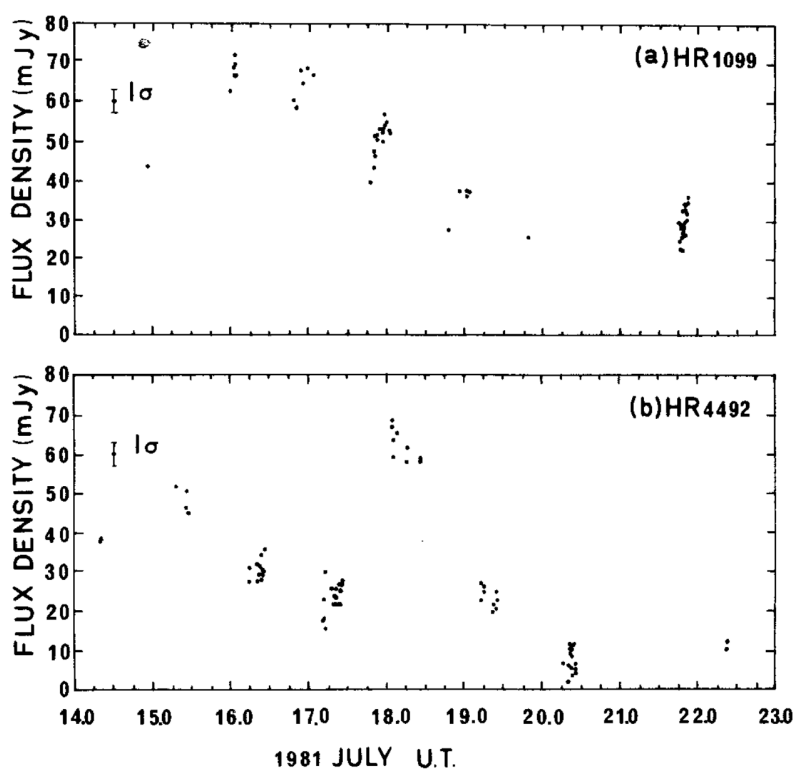

Figure 23 Variations in $5000 \mathrm{MHz}$ flux densities for HD 22468 (a) and HD 101379 (b), observed at Parkes in 1981 (after Collier, Haynes, Slee, Wright and Hillier, 1982: 872).

a quadruple system, the active component being an early $\mathrm{K}$ giant with prominent $\mathrm{H} \alpha$ and $\mathrm{Ca}$ II emission. Of the 21 stars surveyed, three radio detections were made above the $3 \sigma$ level of $10 \mathrm{mJy}$. Two of these were the RS CVn stars HD 22468 and HD 195040, and the third was the early K giant, HD 101379. There was also a possible detection for HD 36705, a dwarf pre-main sequence star. The strongest radio emitters were HD 22468 and HD 101379, whose flux densities varied from 20 to $70 \mathrm{mJy}$ (Fig. 23) and 10 to $65 \mathrm{mJy}$ during the eight-day observing period, with the latter star exhibiting what appeared to be two consecutive radio flares during one 7 -h period, which were accompanied by significant $\mathrm{H} \alpha$ enhancement. The authors noted that HD 101379 was a known X-ray emitter, and the detection of radio emission and radio flaring added "... to the body of evidence for a very high level of chromospheric and coronal activity in this system.... The simultaneous enhancement observed at both $\mathrm{H} \alpha$ and at $5 \mathrm{GHz}$ on July 18 suggests that a non-thermal mechanism ... [is] responsible. Further simultaneous radio observations of this object at different frequencies are needed ..." (Collier, Haynes, Slee, Wright and Hillier, 1982: 878-879).

In fact, Slee was to make further observations of this enigmatic K-type star, but before doing so he and two Radiophysics colleagues began a major research project, which they formally referred to as the 'Australian Radio Star Survey' (ARSS). They were interested in researching the radio emission from a wide range of stars, both binaries and single stars. This project would span six years, draw in a large number of other Australian and overseas collaborators - from both optical and radio fields - and result in the publication of numerous papers, some of a preliminary nature which would report components of the project long before the appearance of the major ARSS report in 1987. 


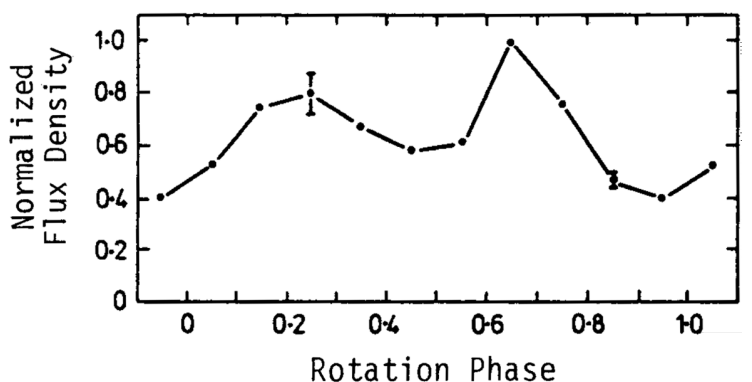

Figure 24 Plot of flux density vs rotation phase for HD 36705 (after Slee et al. 1986: 314).

The first major initiative in the ARSS project occurred in 1982 March and 1983 July-August when Slee and two colleagues used the Parkes Radio Telescope at $5000 \mathrm{MHz}$ to observe 45 different stars. Five definite new detections and three possible detections (pending unequivocal source identifications) were reported; four of these definite detections were RS CVn binaries and the fifth an Am star (HD 30050). The authors noted that the peak $5000 \mathrm{MHz}$ luminosities of three of these RS CVn stars exceeded $10^{10} \mathrm{~W} \mathrm{~Hz}^{-1} \mathrm{sr}^{-1}$, and that an electron-cyclotron maser was a possible emission mechanism (Slee, Haynes and Wright, 1984: 865).

At this point Slee and two of his Radiophysics collaborators joined in an optical-radio investigation of the RS CVn star HD 127535 involving three astronomers from Monash University (Melbourne). In 1984 August optical photometric monitoring was carried out with the 0.4 and 0.6 telescopes at Siding Spring, while the radio observations were made with the Parkes Radio Telescope at 8400 and $22000 \mathrm{MHz}$ (earlier observations at $5000 \mathrm{MHz}$ having been unsuccessful). On this occasion, the star was easily detected at both frequencies, but no correlation was noted between the radio flux densities and the photometric light curve (Innis et al. 1985).

Slee and five Australian colleagues then returned to one of the possible detections recorded in 1981 and in 19821983, the dwarf pre-main sequence star HD 36705, and between 1985 December and 1986 February they carried out a systematic search for radio emission at $8400 \mathrm{MHz}$ with the Parkes Radio Telescope. On this occasion they were particularly successful, and two strong radio flares with peak radio powers of $\mathrm{P}_{8.4} \approx 4 \times 10^{9} \mathrm{~W} \mathrm{~Hz}^{-1}$ were detected, each lasting about three days (Slee et al. 1986: 312; c.f. Budding, Burgess, Chan and Slee, 1991). When the radio data were phase-binned using the 0.514-day rotational period of the star two radio maxima $\sim 180^{\circ}$ apart were noted (see Fig. 24), and subsequent photometric monitoring revealed intensity variations consistent with starspots at these longitudes.

In an attempt to determine the emission mechanism for the source of the microwave radiation, Slee and seven Australian colleagues then turned their attention to the RS CVn star, HD 22468, which was successfully observed in 1981 (when it showed marked flux variability on a short time scale). Further observations were made at $5000 \mathrm{MHz}$ with the Parkes Radio Telescope in 1982 and 1984, together with observations at $8400 \mathrm{MHz}$ and $22000 \mathrm{MHz}$ in 1984, then in 1985-1986 observations were made at $1420 \mathrm{MHz}$ with the Fleurs Synthesis Telescope. Radio detections were made at all frequencies, and simultaneous 1420 and $8400 \mathrm{MHz}$ observations gave a spectral index of $\alpha=-0.1 \pm 0.2$. After comparing their data with models published by other researchers, Bunton, et al. (1986: 317) concluded that a simple gyrosynchrotron model could not explain the radio emission. Other, more complex, models were called for.

It was also at this time that Slee and his Australian colleagues linked up with three US-based astronomers to continue the ARSS project as an international collaboration, and for a 24-h interval on 1985 September 14 they carried out simultaneous observations with the Parkes Radio Telescope at $8400 \mathrm{MHz}$ and with the VLA at 4900 and $15000 \mathrm{MHz}$. All the stars on the observing list had been detected previously, and the aim of this project was to investigate their spectra and polarisation. Most of the stars were RS CVn binaries, but there were also four BY Draconis stars, four unspecified Ca II emitters, one Algol-type eclipsing binary, and one classical dMe flare star. In all radio emission was detected from 14 stars, but the authors noted a striking difference between the power outputs and brightness temperatures of the higher optical luminosity stars and low luminosity stars, which they explained in terms of differences in the surface areas and rotational velocities of the two groups of stars. Meanwhile, the spectra of the different classes of stars exhibited large variations, and only moderate degrees of circular polarisation were detected in the radio emission from just two of the stars (Slee et al. 1988).

In a paper published in the Monthly Notices of the Royal Astronomical Society, Slee and six Australian collaborators then provided a lengthy account of the ARSS Project. Altogether, 153 stars were observed at $5000 \mathrm{MHz}$ and/or $8400 \mathrm{MHz}$ using the Parkes Radio Telescope, including RS CVn binaries, Algol-type binaries, AM Her-type binaries, BY Draconis binaries, FK Comae stars, and unidentified strong [CaII] emitters. Radio emission at $>2$ mJy was detected from $70(46 \%)$ of these stars. Slee et al. (1987b: 659) reported

"... the highest detection rate of 68 percent from the RS CVn group. The Ca II stars and Algol-type binaries yielded detection rates of 44 and 30 percent respectively. Two of the four observed AM-Her-type binaries were detected.

"The maximum powers emitted by the stars at 5.0/8.4 GHz ranged over five orders of magnitude, with a median of $2.5 \times 10^{10} \mathrm{~W} \mathrm{~Hz}^{-1} \ldots$.

"The maximum brightness temperatures had a smaller range of three orders of magnitude, with a median of $3.6 \times 10^{9} \mathrm{~K}$. The brightness temperature distributions of stars in the RS CVn/Ca II classes are not significantly different, but the Algols possess a markedly 
higher median brightness temperature of $1.1 \times 10^{10} \mathrm{~K}$. The AM Her binaries are still higher by at least one order of magnitude....

"The moderate values of brightness temperature of most of the stars, combined with a positive spectral index and generally low polarization, suggest that optically thick gyro-synchrotron emission is the dominant mechanism. However, there are a number of stars, including most of the Algols and the AM Her binaries, with sufficiently high brightness temperatures (sometimes combined with high polarization) that may emit by a coherent process."

In two much shorter papers, but with much longer lists of authors, Wright et al. (1987) and Stewart et al. (1987) provided simple overviews of the ARSS project. These include lists of the participating radio and optical observatories and summarised the observational results. It was also at about this time that Robinson and Slee (1986) penned a more popular review paper about chromospherically active stars, and a particularly useful figure in this paper was an $\mathrm{H}-\mathrm{R}$ diagram showing the positions of RS $\mathrm{CVn}$ and flare stars relative to the Main Sequence (see Fig. 25).

The publication of the ARSS summary opened the way for Slee and his colleagues to write up different aspects of the overall survey. In a short paper, he and nine Australian colleagues were able to report — with some pride — that

"1986 was a vintage year at Parkes for the detection of energetic microwave events from southern activechromosphere stars. We observed extended flaring episodes at $8.4 \mathrm{GHz}$ from HD 32918, HD 36705 (AB Dor), HD 128171 (RV Lib), HD 132742 ( $\delta$ Lib) and HD 196818 (CF Oct); we detected the first recorded strong $22 \mathrm{GHz}$ flares from HD 32918, and the second

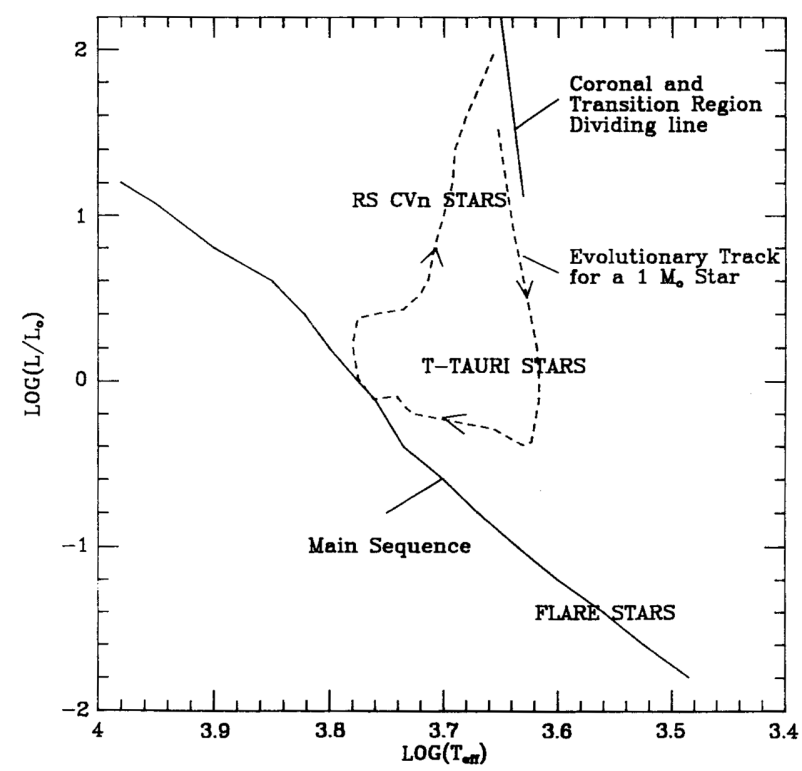

Figure $25 \mathrm{H}-\mathrm{R}$ diagram showing the positions of flare stars and RS CVn stars relative to the Main Sequence (after Robinson and Slee, 1986: 121). recorded $22 \mathrm{GHz}$ event from $\mathrm{HD} 127535$. For some of these events we were able to obtain supplementary flux density measurements at $0.843 \mathrm{GHz}, 1.42$ and $2.29 \mathrm{GHz}$ from the Molonglo Observatory ... Fleurs ... and the Tidbinbilla interferometer respectively, thus enabling us to obtain a limited amount of spectral information [see Fig. 26]. In addition, at $8.4 \mathrm{GHz}$ we made some circular polarization measurements during the stronger phases of flare events." (Slee et al., 1987c: 55).

These six stars comprised two RS CVn binaries, one Algol binary, an FK Comae star, a dwarf pre-main sequence star and a single K-type giant star, and in addition in 1986 there was the first successful detection of radio emission from an AM Her type star, V834 Cen. These discoveries led to further papers documenting the flare event recorded on HD 32918 and the V834 Cen observations.

V834 Cen is a member of a subclass of cataclysmic variable stars that are interacting binary systems with a white dwarf primary and red dwarf secondary. The primary characteristically has a strong magnetic field (30 MG), preventing formation of an accretion disk, so accretion occurs radially at one or both poles of the white dwarf. Radio emission from V834 Cen was recorded at Parkes at $843 \mathrm{MHz}$ between 1986 May 30 and August 25 , reaching a peak flux density of $35 \mathrm{mJy}$, but was variable on time-scales as short as 1 min (e.g. see Fig. 27). Wright, Cropper, Stewart, Nelson and Slee (1988) noted that longer time-scale variations were possibly correlated with binary phase, and they advanced electron-cyclotron masering as the probable emission mechanism.

In contrast, HD 32918 is a K1 IIIp star that belongs to the small but growing FK Comae group of stars. These are rapidly-rotating, single giant or sub-giant stars with

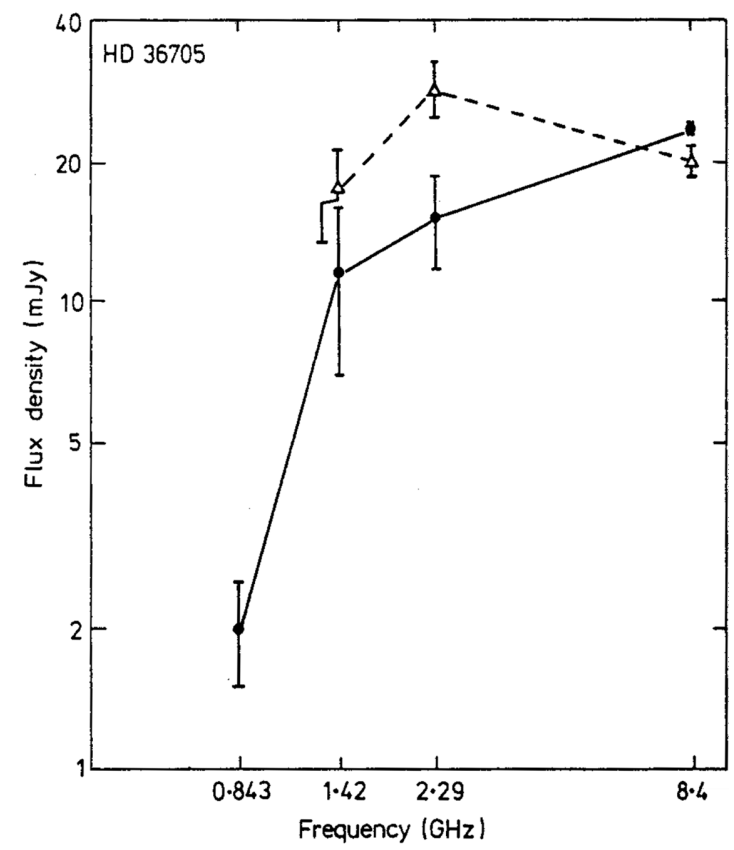

Figure 26 Radio spectra of HD 36705 recorded on 1986 August $23(\triangle)$ and August $26(\bullet)$ (after Slee et al. 1987c: 57). 
strong $\mathrm{Ca}$ II emission lines and erratic $\mathrm{H} \alpha$ emission. The HD 32918 outburst recorded at $8400 \mathrm{MHz}$ with the Parkes Radio Telescope from 1986 May 31 to $\sim$ June 20 was "... the brightest microwave flare yet detected from an active chromosphere star.... At the peak flux the estimated brightness temperature for the $8.4-\mathrm{GHz}$ source, assuming a size of one stellar diameter, was $\sim 3 \times 10^{10} \mathrm{~K} \ldots$ [and the] flare parameters can be explained by gyrosynchrotron emission from an optically thick source." (Slee, et al.

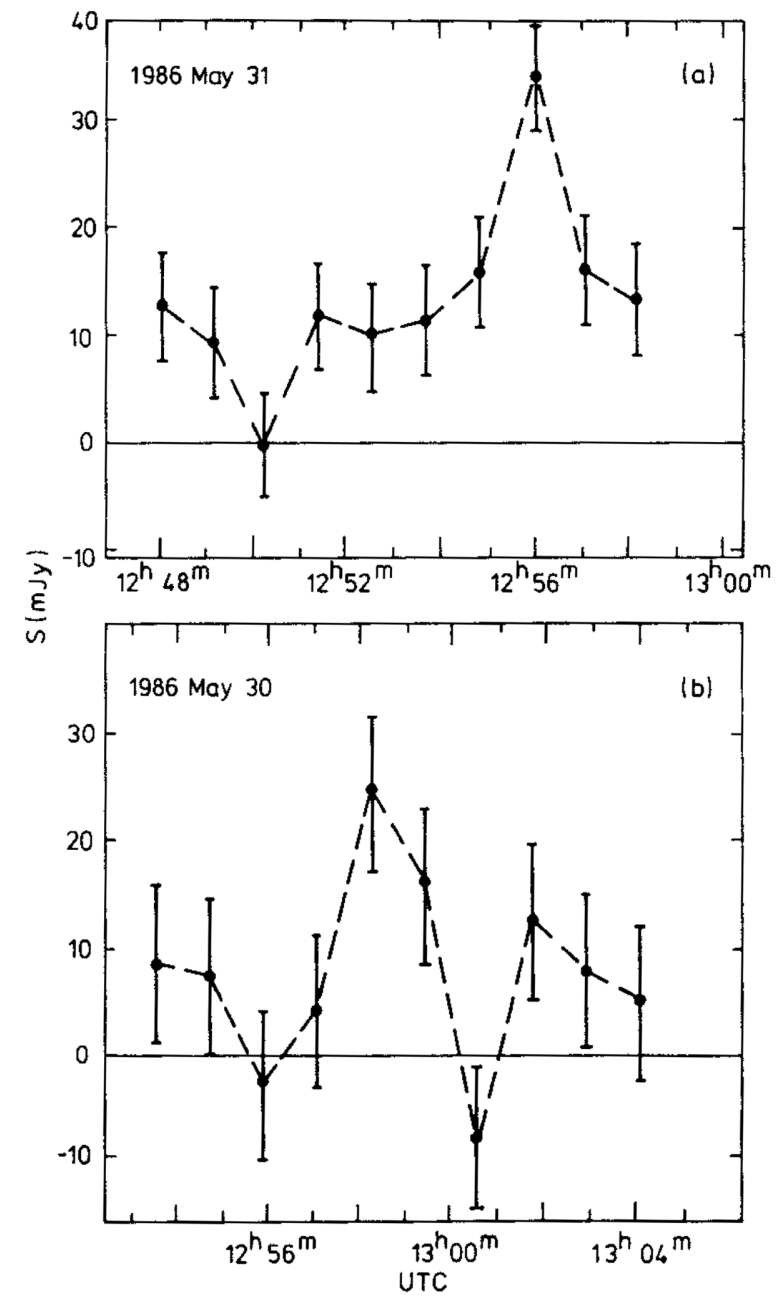

Figure 27 Short-term variability in $843 \mathrm{MHz}$ radio emission from V834 Cen, recorded on two successive days in 1986 (after Wright, Cropper, Stewart, Nelson and Slee, 1988: 321). 1987a: 467). Using a spherically symmetrical source model, the authors went on to compute the magnetic field strength and electron density required to explain the radio flare. At the peak of the flare, it was shown that the radio emission was not composed of a succession of short-duration 'micro-flares' (see Fig. 28).

The successful observations of HD 32918 in 1986 led to further monitoring of this star with the Parkes, Fleurs and Molonglo radio telescopes at 8400, 1420 and $843 \mathrm{MHz}$ respectively in 1987 August, with supporting spectroscopy contributed by the $3.9 \mathrm{~m}$ AAT at Siding Spring. This was the first new project spinning out of the ARSS project. Two successive major radio flares were detected at $8400 \mathrm{MHz}$, each with a duration of about three days, the stronger reaching a peak radio power of $5 \times 10^{12} \mathrm{~W} \mathrm{~Hz}^{-1}$. Enhanced emission was also recorded at $1420 \mathrm{MHz}$, and during the flares the spectrum varied as $\sim v^{1.4}$ and later flattened as they progressed. Optical monitoring during the second flare revealed strong [Ca II] emission (Bunton et al. 1989).

The single detection of one $\mathrm{K}$ giant star at Parkes in 1986 as part of the ARSS project also inspired Slee and his colleagues to embark on a comprehensive radio survey of red giants. These are single rapidly rotating stars, generally of spectral type $\mathrm{G}$ or $\mathrm{K}$, and sometimes with soft $\mathrm{X}$-ray emission. The 82 southern red giants included in the $8400 \mathrm{MHz}$ Parkes survey of 1987 February-1988 July were of spectral type G, K or M and luminosity classes I, II or III, and at distances of $\leq 30 \mathrm{pc}$. Radio emission was detected from 24 stars (29\%), but the median $8400 \mathrm{MHz}$ power and surface flux distributions were two orders of magnitude lower than those of other active radio emitters. Gyrosynchrotron emission from mildly relativistic electrons was favoured as the likely emission mechanism, or, failing that, thermal emission from a cool stellar wind (Slee et al. 1989a: 921-922). In addition to a full account in Monthly Notices, the authors also published a one-page summary in PASA (Slee et al. 1989b), and a follow-up paper on the physical properties of radio-emitting red giants (Jones, Robinson, Slee and Stewart, 1992; c.f. Slee and Budding, 1995).

Also inspired by successful detections of EA eclipsing binaries in the ARSS project, Slee and his Australian and New Zealand colleagues carried out a more intensive investigation of these stars in 1988 using the Parkes Radio

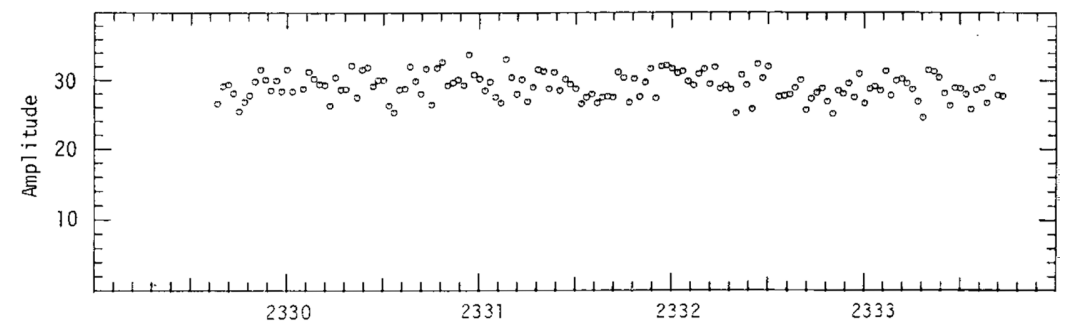

Figure 28 High-resolution observations of HD 32918 at $8420 \mathrm{MHz}$ with the Tidbinbilla Interferometer during the peak of the above flare event. Time is in U.T. There is no evidence of micro-flare activity (after Slee et al. 1987a: 472). 
Telescope at $8400 \mathrm{MHz}$. A literature survey revealed that about half of all EA eclipsing binaries belonged to the EA2-type Algol eclipsing binaries, comprising an early spectral type primary and a late spectral type secondary that has undergone substantial mass loss. Remaining EA eclipsing binaries were of the EA1 type, pairs of main sequence stars earlier than spectral type F5. The 1988 Parkes observations added a further detection to the 14 stars successfully observed previously, seven probably being EA1 binaries and eight EAII binaries. The nature of the radio emission from these binary stars indicated the presence of a moderately strong coronal magnetic field ( $B \approx 10^{2} \mathrm{G}$ ) between the component stars, translating to a surface magnetic field of $\sim 10^{3}-10^{4} \mathrm{G}$ on one or both stars. This was the first compelling evidence for magnetic fields on early-type stars, after the Ap and Bp magnetic stars first detected in 1947 (Stewart et al. 1989: 463).

Meanwhile, Slee and some of his collaborators also found time to carry out analyses of the data accumulated during the ARSS project, producing two interesting papers. In the first, they examined the relationship between $8400 \mathrm{MHz}$ radio luminosity and rotation in 51 late-type $\mathrm{F}$, $\mathrm{G}$ and K-type single and binary stars — including the Sun. Employing a gyrosynchrotron source model, they found "... the main parameter depending on rotation rate is the "filling factor" ... As the rotation speed increases, the scale size of the coronal structures emitting microwave gyrosynchrotron radiation increases, and there is a corresponding increase in the area of the surface covered by intense starspot magnetic fields." (Stewart, Innis, Slee, Nelson and Wright, 1988: 371). In the second paper, Slee and Stewart examined the relationship between microwave surface brightness of 63 chromospherically active ARSS stars and their rotational parameters. They found that when surface flux was used as an activity indicator in lieu of total radio luminosity there was a significant association with rotation (Fig. 29). In testing the relationship on three BYDra stars and the Sun, they found two of the three stars gave consistent results but that the Sun was under-luminous by about a factor of ten (Slee and Stewart, 1989: 129).

In 1990 Slee participated in a multiwavelength investigation of HD 36705 involving the GINGA X-ray satellite, and ground-based optical and radio monitoring. He made the radio observations on 1990 January 10-13 using the Parkes Radio Telescope at $8400 \mathrm{MHz}$ (see Budding, Burgess, Chan and Slee, 1991) and found two emission peaks, at phases 0.0 and 0.5. Vilhu et al. (1993: 474) provided the following explanation for these radio maxima and the associated X-ray and optical observations (including flare events):

"The gyrosynchrotron emissivity depends on the angle $\Theta$ between the line of sight and magnetic field lines in such a way that, the emission peaks at 90 degrees and goes to zero when the angle is small (see Eq. 5). If the spots $\mathrm{A}$ and $\mathrm{B}$ have loop-like magnetic fields and the $8.4 \mathrm{GHz}$ emission comes from the tops of the loops it would then nicely explain the $8.4 \mathrm{GHz}$

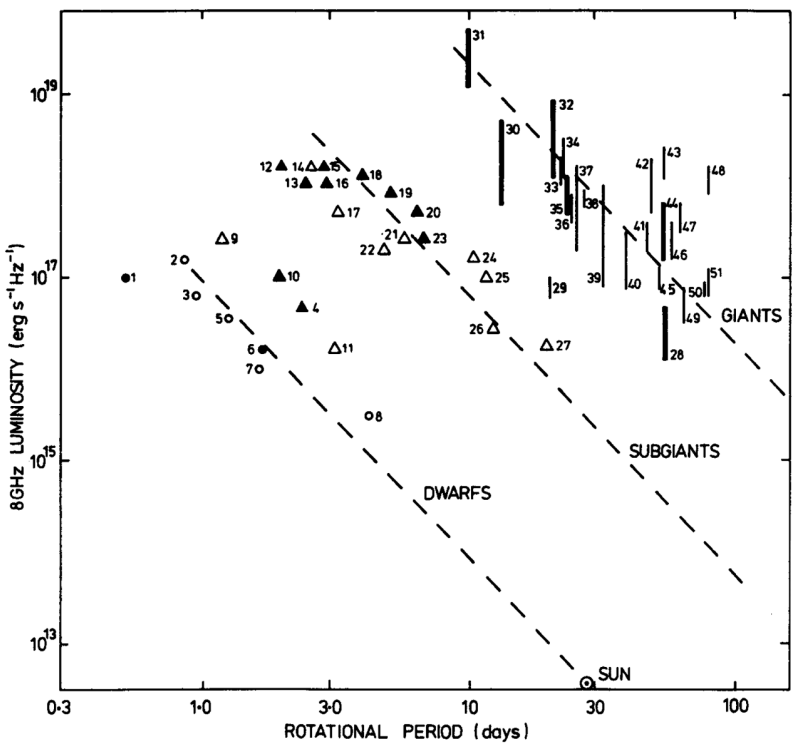

Figure 29 Plot of radio luminosity at $8400 \mathrm{MHz}$ versus rotation period for 51 different late-type stars (after Stewart, Innis, Slee, Nelson and Wright, 1988: 373).

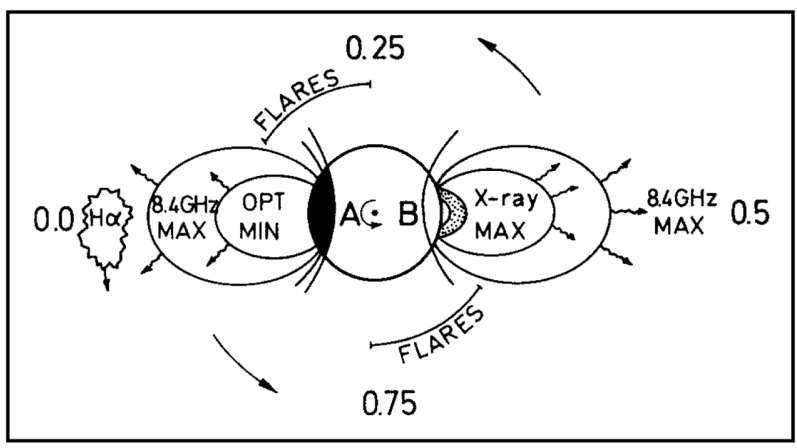

Figure 30 Model proposed to explain the X-ray, optical and radio observations of HD 36705 (AB Dor) made in 1990 January (after Vilhu, et al. 1993: 475).

modulation. The maxima take place when the line of sight is perpendicular to the apexes of the loops."

This scenario is illustrated in Figure 30.

In 1993, Slee and three colleagues returned to an old familiar friend, the RS CVn star, HD 22468, for which radio emission was first detected at Parkes back in 1981. During five consecutive days in September and six days in 1994 August they obtained observations of this star with the ATCA at 1380, 2380, 4800 and $8640 \mathrm{MHz}$ and with the VLA at 4860 and $8440 \mathrm{MHz}$ in order to examine the radio emission processes. They concluded that

"At the higher frequencies $(5-8 \mathrm{GHz})$ the emissions are found to be X-mode gyrosynchrotron emission, as previously thought. At lower frequencies $(1-3 \mathrm{GHz})$ narrow-band bursts of opposite polarized emission are detected. These bursts are probably O-mode plasma emission. Gyrosynchrotron model fits to the radio data imply that in pre-flaring state the emission comes from a region of radius $5 \times 10^{9} \mathrm{~m}$. It requires a highly 


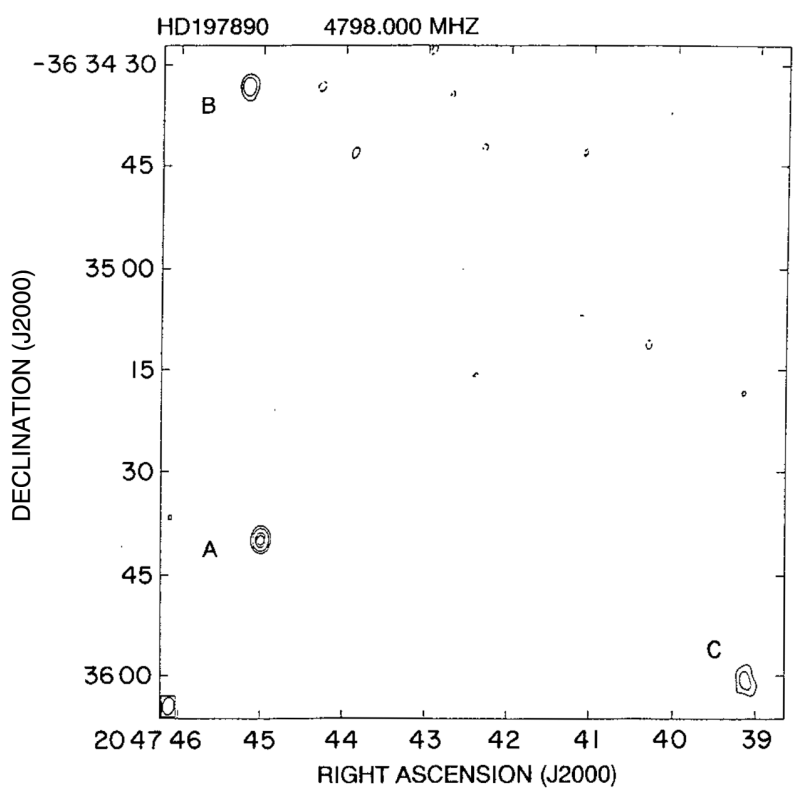

Figure 31 Cleaned $4800 \mathrm{MHz}$ ATCA map of the HD 197890 region. This shows the star (A) and two discrete sources (B and C) (after Robinson, Carpenter, Slee, Nelson and Stewart, 1994: 921).

organized magnetic field of strength ranging from 1 to $13 \mathrm{mT}(10-130 \mathrm{G})$ to explain the radio data from 1.4 to $15 \mathrm{GHz}$. At times of strong flares the emitting region extends to $10^{10} \mathrm{~m}$. The magnetic field is tangled in the outer parts." (Jones, Brown, Stewart and Slee, 1996: 1331).

Slee and his colleagues also used the ATCA in 1992 April to carry out radio observations in conjunction with IUE satellite monitoring of HD 197890. This rapidly rotating K0V single star is a transient UV source, and appears to be a pre-main-sequence object with an age of $\sim 10^{7} \mathrm{yr}$. It has many features in common with single dwarf active stars such as HD 36705. The radio observations were made at 4800 and $8400 \mathrm{MHz}$, and detections of between 1.28 and $2.10 \mathrm{mJy}$ at $4800 \mathrm{MHz}$ and 0.57 and $1.41 \mathrm{mJy}$ at $8400 \mathrm{MHz}$ were recorded. This was the first occasion upon which a 'radio map' of an active star was obtained with the ATCA (see Fig. 31). Meanwhile, the optical monitoring showed strong variable UV emission lines near to saturation levels, but there was no obvious rotational modulation in the UV or the radio flux. Meanwhile, the radio observations showed a level of quiescent emission consistent with other rapidly rotating stars (Robinson, Carpenter, Slee, Nelson and Stewart, 1994: 918).

During the early $1990 \mathrm{~s}$, after his formal retirement, Slee began to collaborate closely with Budding from the Carter Observatory (The National Observatory of New Zealand). Budding's special interest is close binary systems (particularly RS CVn stars and Algol variables), and this is reflected in some of the papers that Slee subsequently published (e.g. see Beasley, Ball, Budding, Slee and Stewart, 1993; Budding, Slee and Stewart, 1992; Budding, Jones, Slee and Watson, 1999a; Budding, Jones, Slee and Watson, 1999b; Budding et al. 1997).

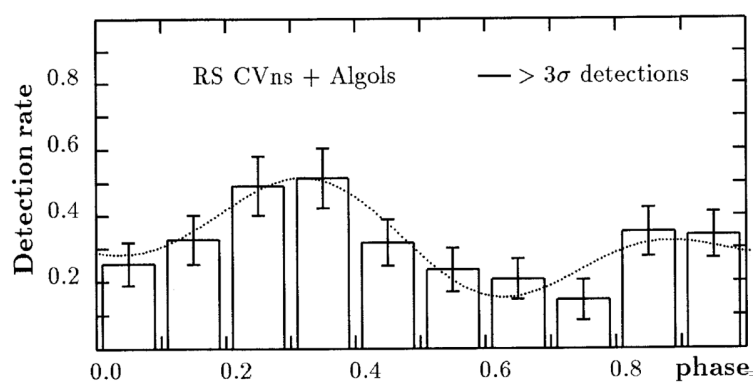

Figure 32 Phase distributions of detections of RS CVn and Algol stars (after Budding, Slee and Jones, 1998: 185).

In an interesting investigation, Slee and Budding used observations made at Parkes in 1989-1990 and with the ATCA in 1989 and 1991 to examine radio emission from a sample of 57 F-type stars situated within 20 pc of the Sun. They reasoned that the detection of radio emission from these stars would be an important discriminator between the competing non-thermal theories: any quiescent or flaring radio emission would almost certainly be a result of the synchrotron process and indicative of magnetic loops in the coronae of these stars (Slee and Budding, 1995: 1063). Possible $8400 \mathrm{MHz}$ emission from 16 of these stars was recorded at Parkes, but there were no detections of these same stars when they were observed with the ATCA at $4800 \mathrm{MHz}$, despite the absence of confusing sources within the Parkes beam. The authors also observed three early F stars with the ATCA in 1992 and 1993, and one of these, the supergiant $\alpha$ Car, was detected (with flux densities of $300 \pm 65$ and $140 \pm 65 \mu \mathrm{Jy}$ at 4800 and $8600 \mathrm{MHz}$. The authors concluded that the $\alpha$ Car emission "... cannot be thermal bremsstrahlung from the $10^{7.2}-\mathrm{K}$ corona of the star but is probably synchrotron emission from a magnetically maintained corona." (ibid.).

Budding, Slee and Jones also carried out a statistical treatment of 8280 individual radio observations of RS CVn stars, EA2 (Algol) eclipsing binaries, and early type binaries (ETBs) made with the Parkes Radio Telescope mainly at $8400 \mathrm{MHz}$. They found that “The Algols' emission appears to increase near conjunction phases, though we cannot clearly distinguish any special property of the Algols' phase-dependent behaviour that is not also shared by the RS CVn binaries [see Fig. 32]. Both these categories' data show a bimodal, phase-dependent pattern to the distribution of detections, suggesting that these binary types share similar underlying physical properties ..." (Budding, Slee and Jones, 1998: 183).

In 1998 August, Slee participated in a multi-wavelength campaign to observe the active Algol binary, KZ Pav. Photoelectric monitoring was achieved with a $40-\mathrm{cm}$ telescope at the University of Southern Queensland's Observatory at Mount Kent, while radio observations were made at 4848 and $4976 \mathrm{MHz}$ using the ACTA. The star was detected (Fig. 33), with flux levels ranging between 0.17 and $1.52 \mathrm{mJy}$, and there was weak evidence of phasedependent variation. The weak level of the radio emission 


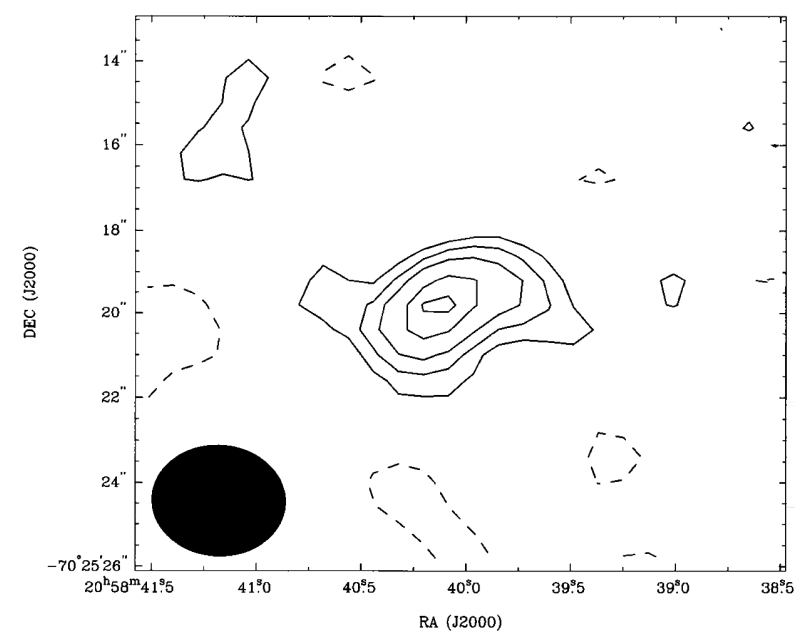

Figure 33 Isophote map of KZ Pav based on observations made in 1998 August using the ACTA (after Budding, Marsden and Slee, 2001: 142).

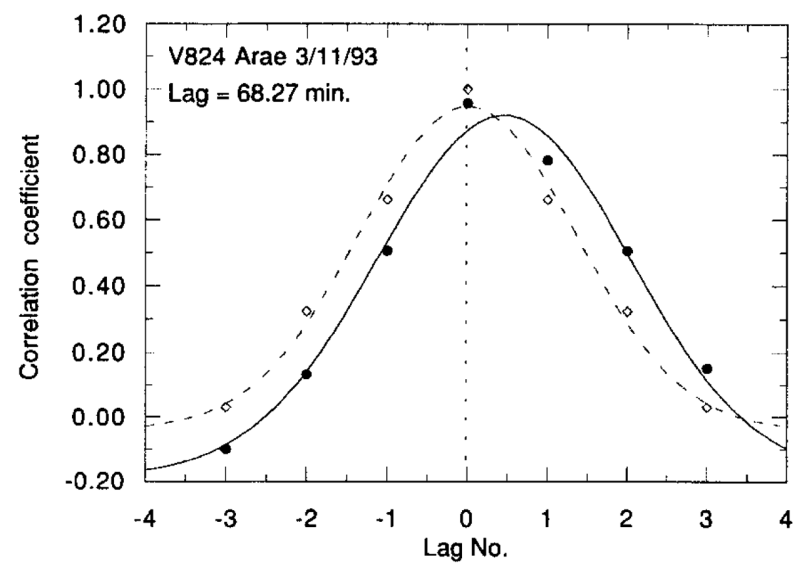

Figure 34 Correlation curves for V824 Arae based on data obtained on 1993 November 3. The full line curve is the correlation between 4800 and $8600 \mathrm{MHz}$ intensity fluctuations (after Budding, Lim, Slee and White, 2002: 41).

suggested a gyrosynchrotron mechanism was involved (Budding, Marsden and Slee, 2001: 146).

Slee is still researching active stars, and his most recent paper at the time of writing (2002 August) was a collaboration with Budding and Taiwanese and American colleagues. In it, ATCA datasets acquired earlier for CF Tuc, V824 Arae (see Fig. 34), HD 22468, HD 37605 and AU Mic were investigated for their auto- and crosscorrelation effects (see Budding, Lim, Slee and White, 2002).

One final paper that includes Slee's name deserves to be mentioned in this section, although the true nature of the object in question was not recognised when it was observed back in 1979. We now know this object, SS 433, to be an exotic 'micro-quasar', a unique binary star lying in our Galaxy. In 1979 July, Slee and two Radiophysics colleagues observed SS 433 with the Culgoora Radioheliograph at 80 and $160 \mathrm{MHz}$, while two Canadian astronomers carried out 1465, 1480, 4885, 15030 and $22485 \mathrm{MHz}$ observations with the VLA. Neither suite of

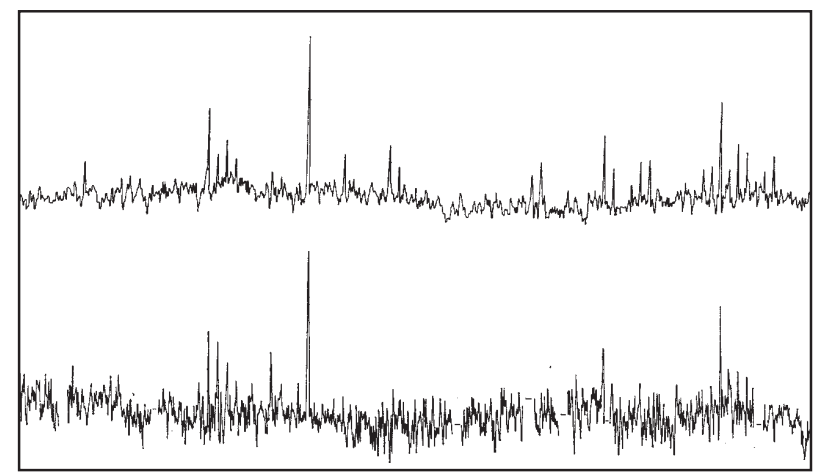

Figure $3580 \mathrm{MHz}$ pulses obtained from PSR $1133+16$ at Culgoora (upper trace) and Parkes (lower trace) over a 2.5-min interval on 1968 May 28 (after Slee, Komesaroff and McCulloch, 1968: 342)

observations helped solve the mystery of SS 433 in 1979 (see Seaquist, Gilmore, Nelson, Payten and Slee, 1980).

\subsection{Investigating the Nature of Pulsars}

For three decades pulsars have been the focus of a major research effort by staff from the Division of Radiophysics and later the ATNF, led by Dick Manchester, and their results have given Australia a formidable reputation in this area (e.g. see Haynes, Haynes, Malin and McGee, 1996). Whilst not part of this consortium, Slee also carried out valuable pulsar research from the late 1960s through into the 1980s, mainly in collaboration with Australian and Indian colleagues.

His first project, carried out with two Australian colleagues, involved observations of PSR 0950+08, $1133+16$ and $1919+21$ from 1968 May 23 to 29, just two months after the earliest Parkes observations of pulsars, and involved one of the key Parkes 'players', Max Komesaroff (see Manchester, 1994). Their aim was to investigate the fluctuations in the amplitude of pulsar emission in order to determine whether these were intrinsic to the source or produced by the ISM and/or the IPM. Simultaneous observations made at $80 \mathrm{MHz}$ with the Parkes Radio Telescope and the Culgoora Radioheliograph (a baseline of $325 \mathrm{~km}$ ) revealed no detectable time displacement between the amplitude variations (Fig. 35). This demonstrated that most of the amplitude variations were imposed at or near the sources and were not caused by interplanetary or interstellar scintillations (Slee, Komesaroff and McCulloch, 1968: 343).

Slee also carried out observations of these same three pulsars at Culgoora later in 1968 in order to (1) investigate temporal changes in the spectrum near $80 \mathrm{MHz}$, and (2) search for periodicities in the pulse amplitudes with periods extending up to one or two minutes. To carry out the spectral observations three filters with bandwidths of $100 \mathrm{kHz}$ and centred on the frequencies of 79.60, 80.00 and $80.40 \mathrm{MHz}$ were inserted in the $1 \mathrm{MHz}$ passband of one of the 48 declination channels of the Radioheliograph, and the array was arranged so as to select alternately the 
linearly and circularly polarised components of the pulsed radiation. Observations of PSR $1919+21$ and $0950+08$ were made on 1968 September 27 and 28 respectively, and both displayed pulses that were highly linearly polarised. Slee (1969: 224) found that "The amplitude changes taking place from one pulse to the next and over intervals of $\lesssim 2 \mathrm{~min}$ were well correlated on the three bands." Furthermore, these spectral observations suggested the presence of two distinct types of amplitude fluctuations: (1) slow changes over intervals of five to ten minutes with a fractional correlation bandwidth of $\Delta \lambda / \lambda \leq 0.005$, and (2) faster changes over intervals of seconds to several minutes with a correlation bandwidth of $\Delta \lambda / \lambda>0.01$ (ibid). Slee also concluded that pulse-to-pulse variations and amplitude changes were not caused by diffraction in the ISM or the IPM.

In order to search for periodicities in pulsar emission, he measured the amplitudes of 800 strong pulses from PSR $1919+21$ and $1133+16$ recorded on 1968 May 24 and 28 respectively, and then subjected these to a power spectrum analysis. However, some derived peaks were not reproducible with other sequences of pulses while others that were required confirmation because they were only a factor of about two above the noise level. Slee (1969: 225) could do little more than conclude that "Further determinations of the power spectra of the amplitude fluctuations from these and other pulsars, preferably from much longer sequences of pulses, may be expected to provide some important evidence bearing on the origin of the pulses." At this time, Slee and two Radiophysics colleagues also used the Parkes Radio Telescope to investigate the positions and periods of five Molonglo PSRs (Morris, Schwarz and Slee, 1970).

Between 1968 May and 1969 March, Slee and Mulhall used the Culgoora Radioheliograph to carry out further investigations of pulsar intensity variations. PSR $0834+06,0950+08,1133+16$ and $1919+21$ were observed until long sequences of strong pulses extending over $>2 \mathrm{~h}$ duration were obtained, and the pulse heights were then transformed to a series of deviations from the line of best fit of the original series. The fluctuations in pulse energy were then subjected to power spectrum analysis, with obvious peaks emerging for all pulsars except $0950+08$. The power spectrum for $0834+06$ contained two peaks, at $f / f_{\mathrm{p}}=0.462$ and 0.075 , with the latter almost certainly the aliased second harmonic of the modulation responsible for the first, stronger, peak (Fig. 36). The power spectrum of PSR $1133+16$ also showed two reasonably well defined peaks, at $f / f_{\mathrm{p}}=0.027$ and 0.229 , although both were considerably broader than the instrumental frequency resolution and may therefore contain several unresolved periodicities. The spectrum of PSR $1919+21$ showed three peaks, a particularly strong one at $f / f_{\mathrm{p}}=0.0032$ and two weaker ones at 0.235 and 0.500 . Slee and Mulhall (1970: 324) cautioned that

"The origins of the secondary periodicities present in 3 of the pulsars studied here ... must remain uncertain

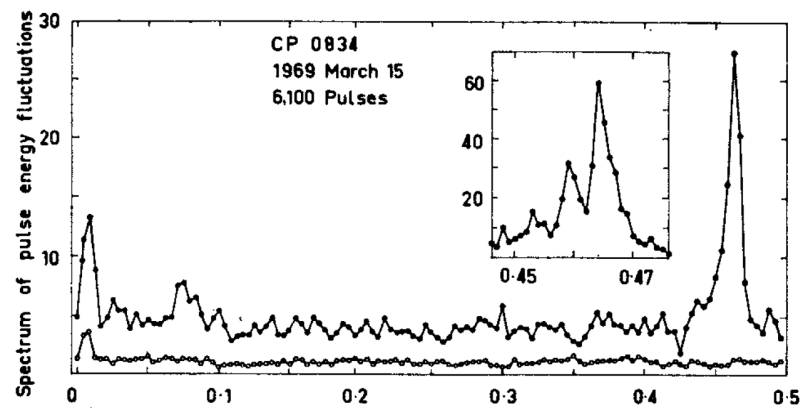

Figure 36 Power spectrum of pulse energy fluctuations for PSR $0834+06$, showing distinct peaks at $f / f_{\mathrm{p}}=0.075$ and 0.462 . The inset shows the fine structure of the latter peak (after Slee and Mulhall, 1970: 323).

until more observations are made to clarify their degree of frequency stability. It is possible that some, if not all, of the apparently wide peaks ... may be resolved into a number of relatively stable frequencies. Some of the periodicities may result from the beating of incommensurate trains of high frequency pulses with multiples of the sampling (pulse-repetition) frequency."

Slee and Mulhall then followed up this paper with another about the secondary periodicities in PSR 0834+ 06, based upon observations carried out at Culgoora on three nights in 1969 March and November and 1970 February. The focus in this instance was on the peak at $f / f_{\mathrm{p}}=0.462$, which stood out on a low-resolution power spectrum based plot on 17280 pulses. However, six high-resolution power spectra showed that this peak was in fact composed of two juxtaposed peaks at $f / f_{\mathrm{p}}=$ $0.4585 \pm 0.0003$ and $0.4624 \pm 0.0003$. Slee and Mulhall (1971: 9) concluded that peaks in the pulse energy spectrum may have considerable fine structure, which may be indirectly related to the presence of subpulses.

Another area of pulsar research which Slee turned his attention to was their energy fluxes, and during 1969 and 1970 he and Hill used the Culgoora Radioheliograph at $80 \mathrm{MHz}$ to measure the average pulse energies of 21 pulsars located between declinations $+34^{\circ}$ and $-45^{\circ}$. Ten of the 21 pulsars were detected with certainty, and upper limits to the average pulse energy were given for the remaining pulsars. Slee and Hill (1971: 442-443) found that the average pulse energy for a typical pulsar could vary by a factor of five between successive observing runs. They also suggested that pulse energy variations over time intervals of days and months were most likely originated at the pulsar. They concluded their paper by comparing some of their results with those obtained by other Radiophysics staff members.

With the further development of the Culgoora Radioheliograph, Slee was able to pursue his study of pulsar flux densities at 80 and $160 \mathrm{MHz}$ (Fig. 37), this time in collaboration with two Indian colleagues. Between 1979 April and 1980 November he observed 74 different pulsars, detecting $44(57 \%)$ and recording upper limits to the flux densities for the remaining 30 . As a result 


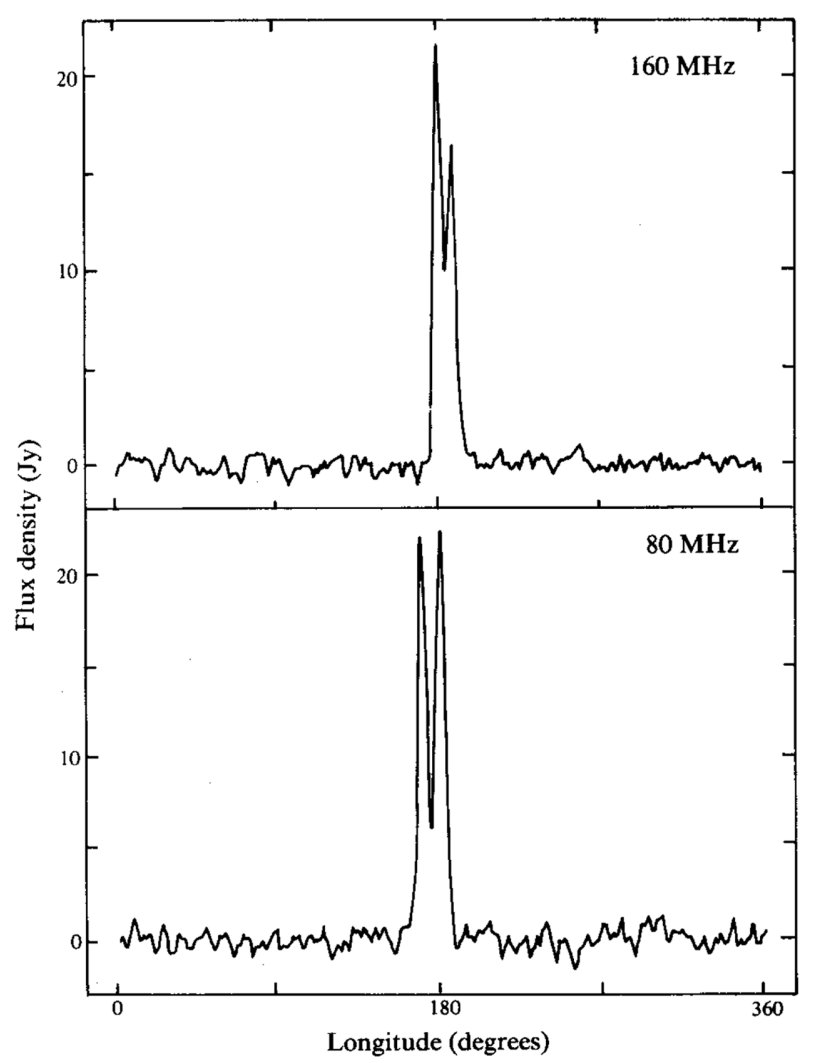

Figure 37 Quasi-simultaneous averaged pulse profiles for PSR $1133+16$ at 80 and $160 \mathrm{MHz}$ (after Slee and Mulhall, 1970: 323).

of this study, Slee, Alurkar and Bobra (1986: 112-113) reported:

“(i) a progressive flattening of the radio spectrum from high to low frequencies,

(ii) no relation between metre-wave spectral index and other measured pulsar parameters,

(iii) most pulsars show significant flux variations on a month-to-month time scale,

(iv) flux variations are well correlated at 80 and $160 \mathrm{MHz}$,

(v) flux variability is inversely correlated with the intrinsic property of dispersion measure and possibly with the intrinsic period derivative [Fig. 38]."

Slee et al. also found that "The form of the radio spectrum is closely connected with the generating mechanism and perhaps with the transmission properties of the pulsar magnetosphere.... [and] The significant relation between flux variability and dispersion measure is probably due to slow scintillation in the interstellar medium." (Slee, Alurkar and Bobra, 1986: 113). They ended the paper by stressing that a more comprehensive observing programme was warranted in order to examine further fundamental relations between pulsar characteristics and parameters of the ISM, but that alternative instrumentation would have to be found given the closing of the Culgoora Radioheliograph. Russians astronomers, in particular,

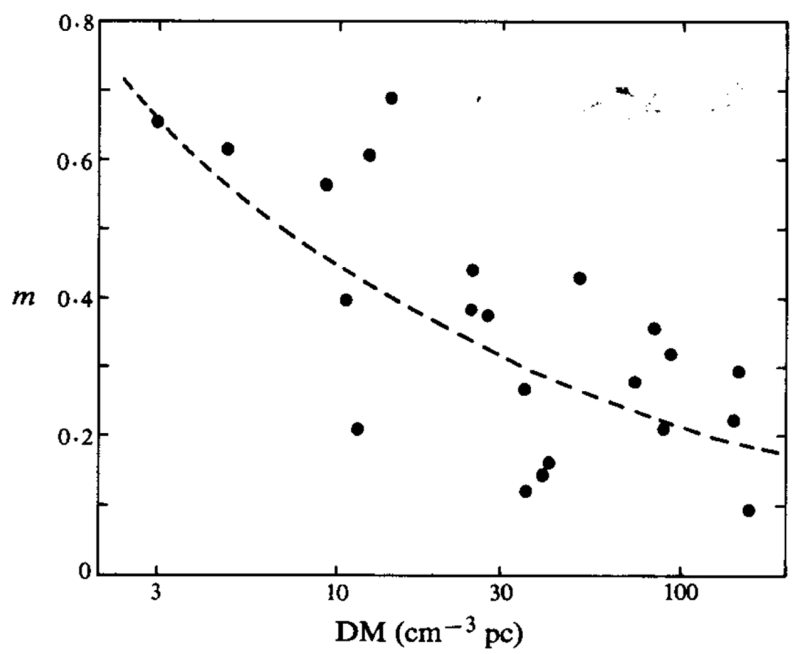

Figure 38 Plot of modulation index $(m)$ versus the dispersion measure (DM) for flux variations in 22 pulsars. The dashed curve is the fitted power-law regression line (after Slee, Alurkar and Bobra, 1986: 111).

subsequently took up this challenge (e.g. see Malofeev et al. 2000).

This, however, did not prevent Slee and his two Indian colleagues from producing one last Culgoora-based paper on pulsar characteristics. In this study they attempted to determine intrinsic pulse widths from the averaged profiles of 24 pulsars observed at 80 and $160 \mathrm{MHz}$ in 1979 and 1980. Pulse widths to half-power points $W_{50}$ were tabulated for all pulsars, and no evidence was found for pulse-widening due to interstellar scattering. They then combined their Culgoora pulse-width estimates with data published by other investigators in order to obtain pulsewidth spectra up to $10.7 \mathrm{GHz}$, and found that these all followed a power-law form but possessed a wide range of slopes (for one example see Fig. 39). The authors then examined the relationship between spectral index, $\beta$, and a range of pulsar parameters, finding a possible correlation only with the rate of loss of kinetic energy, but perhaps the most unexpected result of their study

"... has been to establish a connection between the spectral width of the radio beam and the high-frequency flux spectral index.... [Although the] observed median pulse-width spectral index of $\beta=-0.16$ is close to the suggested theoretical dependence ... there are very significant departures which cannot be explained by the simple [dipolar magnetic field] model ..." (Slee, Bobra and Alurkar, 1987: 585).

Other astronomers have since confirmed the frequency dependence of pulse widths, but as yet no simple explanation for this feature has been proposed (D. Manchester, personal communication, 2002).

\subsection{Sagittarius A and Other Galactic Sources}

The last radio telescope that Slee was involved in at Dover Heights before the radio astronomy program closed down 


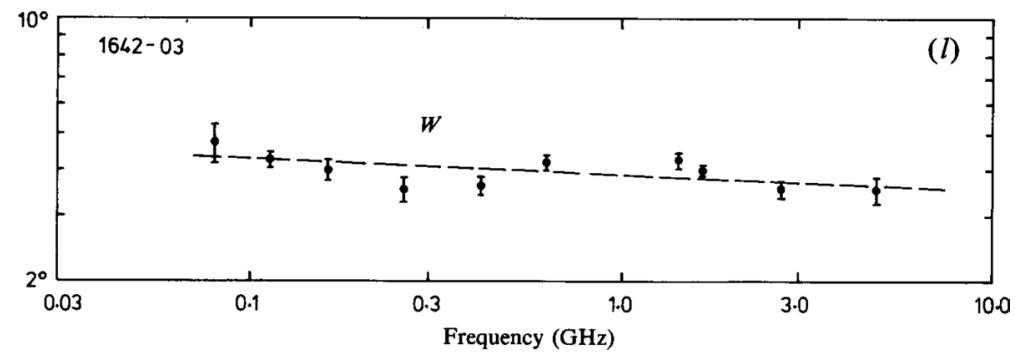

Figure 39 Plot of profile width $\left[{ }^{\circ}\right]$ versus frequency $[\mathrm{GHz}]$, showing the pulse width spectrum of PSR 1642-03 (after Slee, Bobra and Alurkar, 1987: 577).

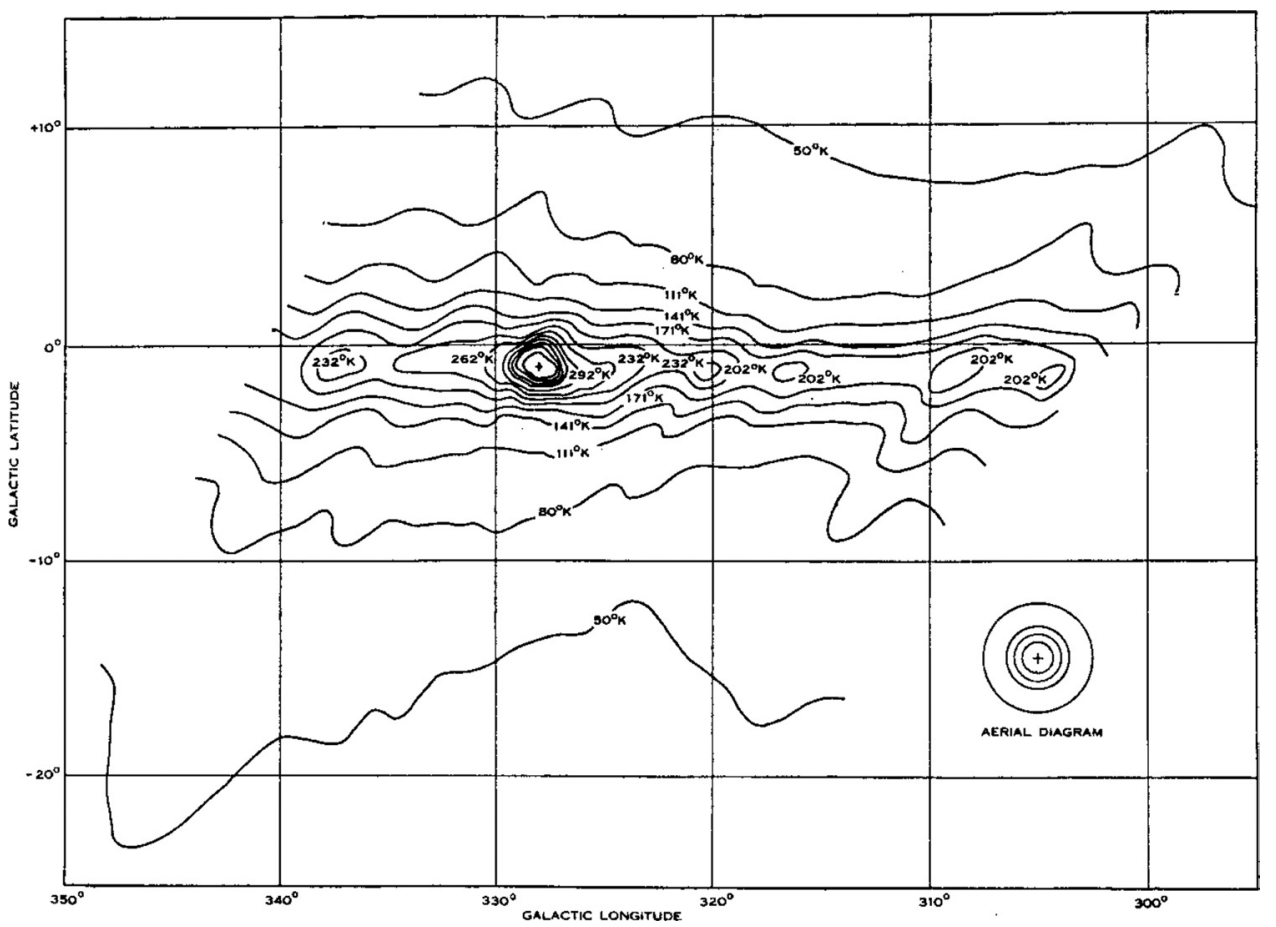

Figure 40 Map of the Sgr A published by McGee, Slee and Stanley (1955: 356).

there was the famous 'hole-in-the-ground' antenna. The construction of this $21.9-\mathrm{m}$ diameter parabolic depression as a lunchtime project and its subsequent expansion to $24.4 \mathrm{~m}$, complete with precision concrete surface, has recently been documented by Orchiston and Slee (2002d). In 1951-1952, Bolton, Westfold, Stanley and Slee (1954) carried out a preliminary survey of the Galactic Centre region at $160 \mathrm{MHz}$, and published a crude map, but this was improved on markedly when they and McGee were able to survey the Galactic plane at $400 \mathrm{MHz}$ with the newly completed 24.4-m antenna (McGee, Slee and Stanley, 1955). Their isophote map is reproduced here as Fig. 40, and the conspicuous discrete source at $l=328^{\circ}$ and $b=-1^{\circ}$ is Sgr A. Its identification with the Galactic Centre was an important milestone for international radio astronomy.

Given this early association with Sgr A, it is no surprise that Slee returned to this source in later years as improved instrumentation came to hand, first with the Mills Cross (e.g. see Fig. 1 in Mills, Hill and Slee, 1958), and then in 1973 with the Culgoora Radioheliograph; meanwhile, in the interim, others from Radiophysics observed this source with the Parkes Radio Telescope. Slee's Culgoora observations were made in collaboration with Dulk. Observing at $160 \mathrm{MHz}$ with a 1.9 arc min beam, they recorded a small, compact, possibly unresolved source, which was apparently superimposed on an extended component $\sim 8$ arc min in diameter. They identified the compact source with Sgr A East (Dulk and Slee, 1974: 33). Using their own observations and those of other observers at different frequencies, they were able to construct a spectrum of this source which showed a rapid decline below $\sim 200 \mathrm{MHz}$. This was explained in terms of absorption in the ISM (see Section 2.11).

By this time the morphology of the Sgr A source and its various components was starting to become complicated and confusing, but this situation was rapidly exacerbated with the advent of the VLA and then VLBI, and three principal components were identified: Sgr A East, a nonthermal shell-shaped source; Sgr A West, a thermal source surrounding the Galactic Centre, and with three different sub-components; and $\operatorname{Sgr} \mathrm{A}^{*}$, the presumed nucleus of 


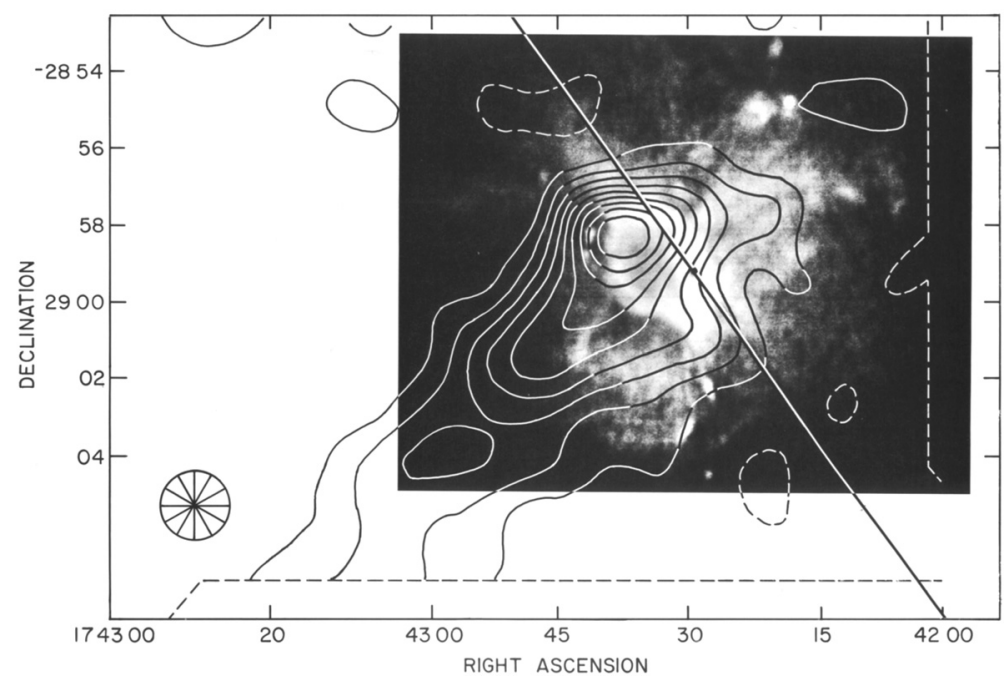

Figure $41160 \mathrm{MHz}$ isophote map showing part of the extended narrow filamentary feature, superimposed on the $1400 \mathrm{MHz}$ VLA map of the region. The oblique line shows the orientation of the Galactic plane, and the black dot indicates the position of the Galactic Centre (after Yusef-Zadeh, Morris, Slee and Nelson, 1986a: Plate L3).

our Galaxy (see Ekers, van Gorkom, Schwarz and Goss, 1983).

The next occasion upon which Slee observed this region was in 1980 and 1982 as part of a large VLBI collaboration that used the Parkes Radio Telescope and the 64-m Tidbinbilla antenna to investigate the Galactic Centre source, Sgr A*, at 2300 and $8400 \mathrm{MHz}$. At the lower frequency no fringes were detected, which for an assumed flux density of $0.85 \mathrm{Jy}$ indicated an angular size of $>100$ mas for a circular Gaussian source. In contrast, the $8400 \mathrm{MHz}$ observations revealed an asymmetrical compact source with a 17.4 mas major axis, an axial ratio of 0.53 , and a position angle of $82 \pm 6^{\circ}$. If the distance to the Galactic Centre was taken as $10 \mathrm{kpc}$, then this translated to apparent dimensions of $170 \times 90$ a.u. for the $\mathrm{Sgr} \mathrm{A}^{*}$ source (Jauncey, et al. 1989).

In 1984 Slee joined with American and Australian colleagues to map the high-resolution large-scale structure of the Sgr A region at 160 and $327 \mathrm{MHz}$ using the Culgoora Radioheliograph. To their surprise, the $160 \mathrm{MHz}$ observations revealed a narrow large-scale filamentary feature lying along the rotational axis of the Galaxy, approximately perpendicular to the Galactic plane, and extending $\sim 30$ pc from the Galactic Centre (see Fig. 41). Constraints on its spectral index indicated that it was nonthermal. On the basis of its location and orientation, Yusef-Zadeh, Morris, Slee and Nelson (1986a) suggested that this filament was linked to small-scale structure in Sgr A West, and that it may be a jet similar to but on a much smaller scale than those associated with some active galaxies. This new feature, or jet, was not present on the $327 \mathrm{MHz}$ map of the region.

Meanwhile, an emission feature associated with the Galactic Centre Arc and visible at $160 \mathrm{MHz}$ was written up by Yusef-Zadeh, Morris, Slee and Nelson (1986b) after further data were accumulated through VLA observations.

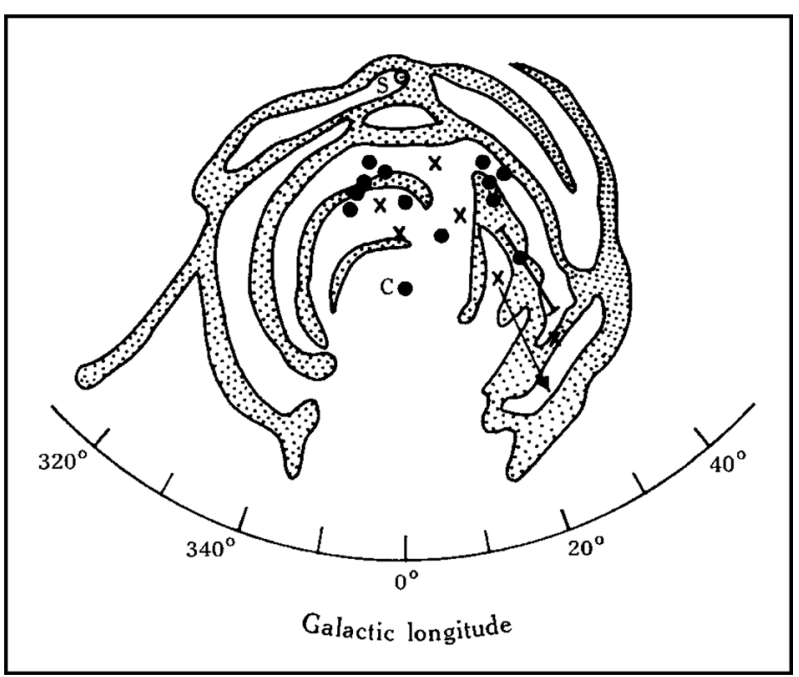

Figure 42 Schematic diagram showing the HI structure of the Galaxy, with the position of the Sun and the Galactic Centre source, Sgr A, marked by S and C. The positions of the observed SNRs are indicated; filled circles and crosses represent absorbed and unabsorbed SNRs respectively (after Dulk and Slee, 1972: 437).

This was to be Slee's last paper about the Sgr A region, and at the time he could not help but be impressed by the way in which knowledge of this area had changed since he first began exploring the Galactic Centre all of 30 years earlier.

Although Sgr A was Slee's main Galactic non-stellar preoccupation, it was not his exclusive interest. He also found time to carry out the first detailed radio survey of southern planetary nebulae (Slee and Orchiston, 1965), to search for radio emission from the Sco X-1 source (Slee and Higgins, 1971b) and from the young open cluster IC 2391 (Lim, Slee and Stauffer, 1996b), and in collaboration with Dulk to investigate features of Galactic SNRs (Dulk and Slee, 1975; Slee and Dulk, 1974). 


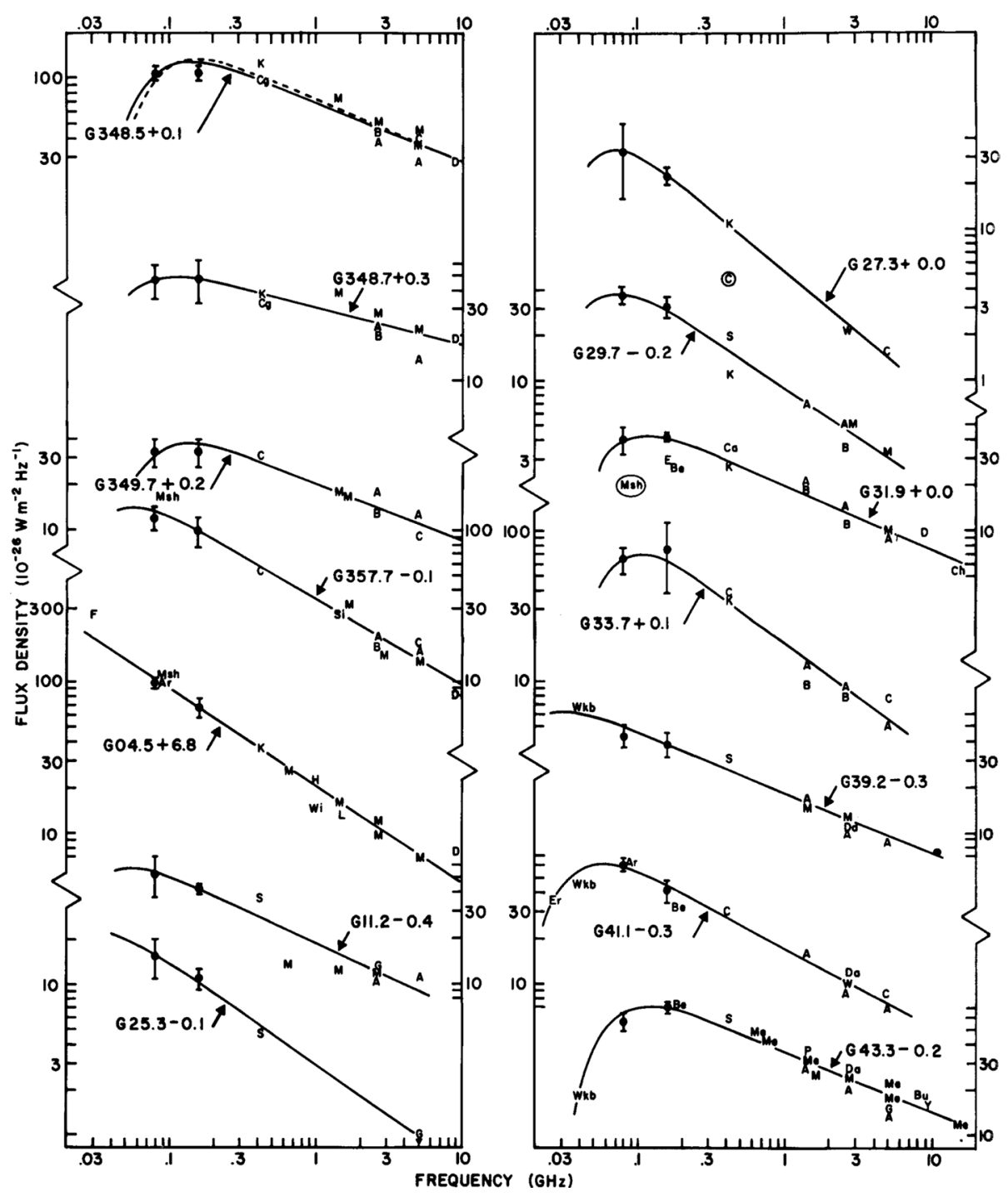

Figure 43 Spectra of $14 \mathrm{SN}$ remnants and least-squares fits, showing spectral turn-overs at $\sim 100 \mathrm{MHz}$ (after Dulk and Slee, 1975: 64)

\subsection{The Nature of the ISM}

After his earlier forays into the IPM, it is no surprise that Slee was also interested in properties of the ISM, and he began active research in this field during his English sojourn at the Royal Radar Establishment. Using a $38-\mathrm{MHz}$ interferometer with a $16200 \lambda$ baseline, he measured the angular sizes of several QSOs and radio galaxies. These observations showed that "For the sources of high latitude, where the total path length is unlikely to exceed $100 \mathrm{pc}$, the average electron density is less than 0.1 electrons $\mathrm{cm}^{-3}$ and/or the scale size is greater than $10^{-3} \mathrm{pc}$; at low galactic latitudes, where the path length may be an order of magnitude greater, the above limits are conservative." (Slee 1967).

During the early 1970s, Slee and Dulk used $80 \mathrm{MHz}$ Culgoora Radioheliograph observations of Galactic SNRs to investigate the ISM. In their first paper they observed 20 different sources (Fig. 42) and evidence of line-of-sight electron density of $0.1-0.5 \mathrm{~cm}^{-3}$, a value that required a greater ionising flux than occurs in the vicinity of the Sun (Dulk and Slee, 1972: 429).

In a later paper, reporting 80 and $160 \mathrm{MHz}$ Radioheliograph observations of 14 Galactic SNRs, Slee and Dulk found the spectra of more than half the sources exhibited a turnover at about $\sim 100 \mathrm{MHz}$ (Fig. 43 ) which they attributed to free-free absorption taking place in the inner regions of the Galaxy. They posited various explanations for this absorption:

"If the absorbers are HII regions, they must be large $(\gtrsim 20 \mathrm{pc})$ and tenuous $\left(n \approx 10 \mathrm{~cm}^{-3}\right)$. However, in the case of Sgr A, 15.5-GHz continuum measurements, together with low-frequency absorption measurements, lead to a limit of $T_{\mathrm{e}} \lesssim 2000 \mathrm{~K}$. This limit casts doubt on the HII absorption hypothesis, at least for Sgr A.

"Other possible absorbers, cold clouds with $T_{\mathrm{e}} \lesssim 100 \mathrm{~K}$, are features of both steady-state and time-dependent models of the interstellar medium. The former model 


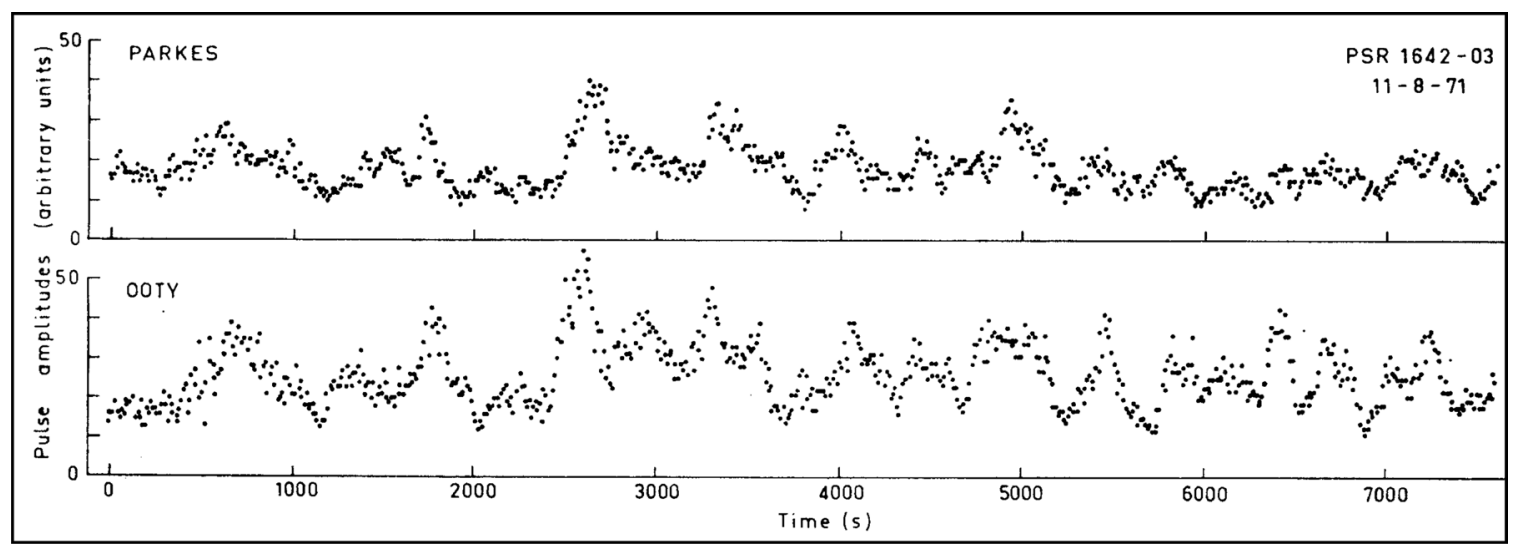

Figure 44 Plots of smoothed pulse amplitudes for PSR 1642-03 obtained at Parkes and Ooty on 1971 August 11 (after Slee et al. 1974: 36).

is barely consistent with the observations because extremes of temperature, ionization fraction, and ionization rate are required. The time-dependent model, in which large portions of space consist of cold, partly ionized, moderate density gas, seems to be most compatible with the observations." (Dulk and Slee, 1975: 67).

In a later study, carried out with an Indian collaborator, Rao and Slee used $327 \mathrm{MHz}$ Ootacamund (Ooty) Radio Telescope and $1420 \mathrm{MHz}$ Parkes Radio Telescope observations of 42 radio sources near the Galactic plane $\left(|b|<3^{\circ}\right)$ to investigate scattering in the ISM. While eight sources showed interplanetary scintillations at $1420 \mathrm{MHz}$, only one of these exhibited this feature at $327 \mathrm{MHz}$. Rao and Slee suggested that interstellar scattering (ISS) was responsible for the widespread absence of interplanetary scintillations at this lower frequency, and used this interpretation to set lower limits for the ISS angle of 0.65 arc sec for 1748-253 and 0.7 arc sec for 1808-209. Both sources were located in the direction of the Galactic Centre $\left(3^{\circ}<l<10^{\circ}\right.$ and $\left.|b|<1^{\circ}\right)$, and the derived ISS angles were an order of magnitude larger than expected from observations at high latitudes, suggesting that there is a region of enhanced scattering in the inner parts of our Galaxy (Rao and Slee, 1988: 861).

Over the years, Slee also used observations of pulsars to investigate the ISM. In his first such study, he and two Radiophysics colleagues observed PSR 0833-45 at $80 \mathrm{MHz}$ with the Culgoora Radioheliograph, confirming the view of Ables et al. (1970) that the spectral turnover in the pulsed component of the emission at higher frequencies was due to scattering from ISM electron density inhomogeneities (Higgins, Komesaroff and Slee, 1971: 75).

In 1971 August Slee together with Radiophysics and Indian colleagues also carried out simultaneous 326.5 $\mathrm{MHz}$ observations of seven pulsars using the Parkes Radio Telescope and the $530 \times 30 \mathrm{~m}$ Ooty radio telescope. With the exception of one source, all of the pulsars showed intensity fluctuations that could be ascribed to interstellar scintillations (e.g. see Fig. 44). Slee et al. reported that
“... it appears from the lack of high instantaneous correlation between the stations, especially for the more highly dispersed pulsars, that the pattern scale size at $326.5 \mathrm{MHz}$ is less than the $8000-\mathrm{km}$ spacing.

"The effect of intrinsic variability on a time scale similar to that of interstellar scintillation is to shift the crosscorrelation peak to smaller lags and increase the peak cross-correlation coefficient. Many pulsars may possess this type of variability ... with the result that measurements such as those of Lang \& Rickett (1970) and Galt \& Lynne (1972), based on the position of the crosscorrelation peak, may have seriously over-estimated the drift velocity and consequently the scale size of the pattern." (Slee et al. 1974: 45).

To further complicate matters, Slee et al. (1974: 46) noted that the emission received from PSR 1642-03 on 1971 August 11 could not be interpreted in terms of a simple model of the ISM. They suggested that the line of sight to the pulsar was probable occupied by many diffraction 'screens', each moving with its own peculiar transverse velocity.

In yet another attempt to investigate the ISM, Slee and two Radiophysics colleagues observed eleven different pulsars at 80 and $160 \mathrm{MHz}$ with the Culgoora Radioheliograph, with a view to obtaining an accurate expression of the relationship between $\tau$ (delay time) and DM (dispersion measure) and using this to test the hypothesis that the scattering properties of the ISM are invariant within $\sim 15 \mathrm{kpc}$ of the Sun. Their conclusions included the following statements: "Our investigations of the $\tau-\mathrm{DM}$ relationship using our own measurements and all available data from other sources shows $\tau \propto \mathrm{DM}^{3.5}$. There is little observational justification for earlier suggestions that the scattering properties of the interstellar medium within a few hundred parsecs of the Sun are qualitatively different from those at much greater distances.... [and] The steep slope of the $\tau$-DM relationship is not inconsistent with present-day models for scattering in the interstellar medium." (Slee, Dulk and Otrupcek, 1980: 105).

Slee's last paper on pulsars and the ISM was a collaboration with Indian colleagues and employed data on 


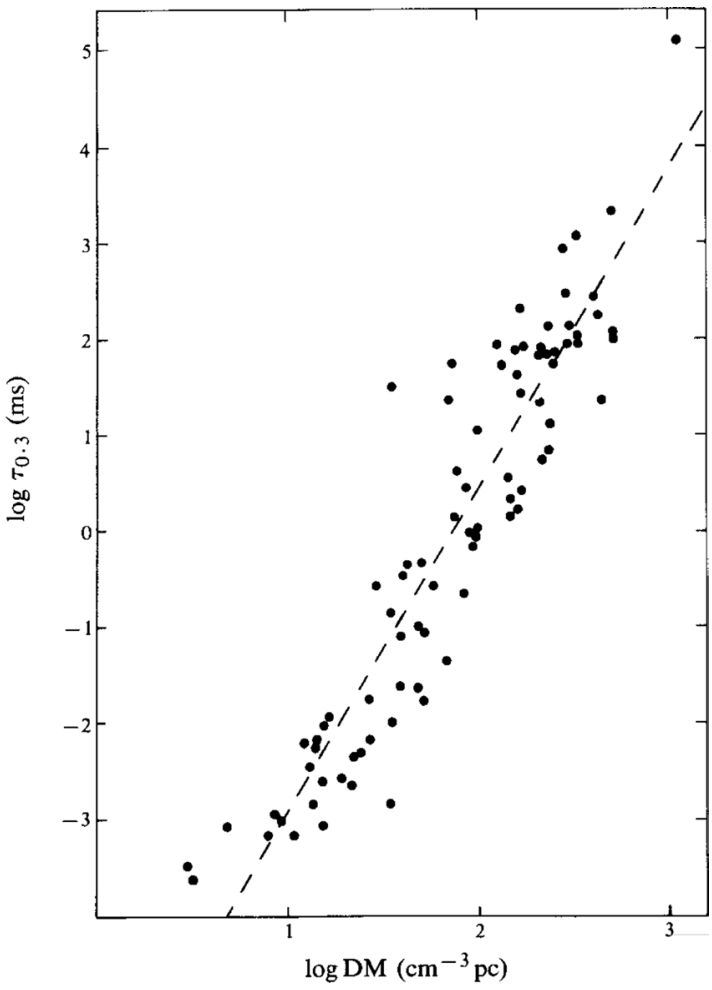

Figure 45 Plot of pulse broadening $\tau_{0.3}$ versus dispersion measure (DM) for 85 pulsars and the least-squares line of best fit (after Alurkar, Slee and Bobra, 1986: 440).

pulse-broadening for 33 pulsars observed between 1978 and the end of 1980 with the Culgoora Radioheliograph at 80 and $160 \mathrm{MHz}$ and with the Parkes Radio Telescope at $410 \mathrm{MHz}$. To these were added published data on 52 other pulsars. Their investigation of average interstellar turbulence levels along 85 different paths in the Galaxy revealed a significant relationship between turbulence level, DM, heliocentric distance, galactocentric distance, $l$ and $b$. These results were also consistent with a distribution of turbulence that peaks near the galactic centre and extends out to beyond the Sun (Alurkar, Slee and Bobra, 1986: 434). Although the $\tau$-DM relationship was basically unchanged (Fig. 45), the authors noted that the above conclusion replaced and expanded on that reported earlier by Slee, Dulk and Otrupcek (1980).

\subsection{Long-baseline Interferometry and Radio Galaxies}

In 1964 Slee accepted a two-year contract appointment at the Royal Radar Establishment in England, and it was during this visit that he became involved in longbaseline interferometry of galaxies (including the enigmatic QSOs).

The first project in which he participated was an RREJodrell Bank collaboration that sought to establish upper limits to the angular sizes of four QSOs and one unidentified source, which were observed at $1420 \mathrm{MHz}$ with the Jodrell Bank Mark 1 radio telescope and a 25-m RRE antenna, a baseline of $127 \mathrm{~km}$ (Adgie et al. 1965). Since one of these sources was unresolved at any hour angle its angular size was $<0.1$ arc sec, but the other four sources were partially resolved at some hour angles, indicating their maximum angular dimensions (assuming Gaussian source models) were between 0.12 and 0.16 arc sec. The QSO 3C 273 was also observed, and the radio flux of the B component was shown to have increased by $\sim 30 \%$ in three years. Furthermore, the B component was found to consist of two elements, each $<0.1$ arc sec in angular size and separated by $\sim 0.4$ arc sec.

Soon after this, Slee was involved in another collaboration between the RRE group and colleagues at Jodrell Bank, looking at the angular sizes of 46 different extragalactic sources, a mix of QSOs and radio galaxies. Interferometer observations were carried out at 1420, 2727 and $5000 \mathrm{MHz}$ using an antenna at the RRE and the Mark II radio telescope at Jodrell Bank. At the highest frequency the maximum effective baseline was $>2 \times 10^{6} \lambda$, giving an optimal resolution of 0.025 arc sec. Most sources observed at 1420 and $2727 \mathrm{MHz}$ were unresolved, and this was also the case for the four sources (three QSOs and a Seyfert Galaxy) observed at $5000 \mathrm{MHz}$. The authors were surprised that so many sources had angular dimensions of $<0.05$ arc sec, and they concluded that this may have been a general feature of most QSOs, and that "Any theory to account for these sources must explain the large radiating power being generated from such a small volume." (Palmer et al. 1967). In 1966 August and November Slee and one of his Jodrell Bank colleagues then extended the Malvern-Jodrell Bank long-baseline interferometry project to include observations at $38 \mathrm{MHz}$. The aerials were separated by $127 \mathrm{~km}$, giving a maximum effective baseline of $16200 \lambda$. Thirty-four different radio sources were observed, and amplitude fluctuations associated with ionospheric and IPM scattering were recorded (Slee and Wraith, 1967).

After returning from England it was some time before Slee became involved in another long-baseline interferometry project, and when he did it was as part of the Australian-American-South African SHEVE consortium. SHEVE, the Southern Hemisphere VLBI Experiment, was carried out in 1982 April-May and used a temporary southern hemisphere VLBI array, with antennas at Parkes (64 m), Fleurs (13.7 m), Tidbinbilla (64 m), Hobart $(13.7 \mathrm{~m})$ and Alice Springs $(9.1 \mathrm{~m})$ in Australia and at Hartebeesthoek $(26 \mathrm{~m})$ in South Africa. All six antennas operated at $2290 \mathrm{MHz}$, with Parkes and Tidbinbilla also using $8420 \mathrm{MHz}$. The maximum baseline of the array, from eastern Australia to South Africa, was $\sim 9600 \mathrm{~km}$. The astrophysical aim of the SHEVE Project was to investigate the fine-scale angular structure of 29 southern hemisphere radio sources. Most of these were QSOs or radio galaxies $(0.0015<z<3.780)$, but the list also included the Vela pulsar, the Sgr A* source and Cir X-1 (a flaring binary stellar system). In addition to these astronomical targets, the SHEVE Project also had astrometric and geodesic goals.

In a paper listing 29 authors, of whom Slee is one, Preston et al. (1989) provided thumbnail sketches for the 


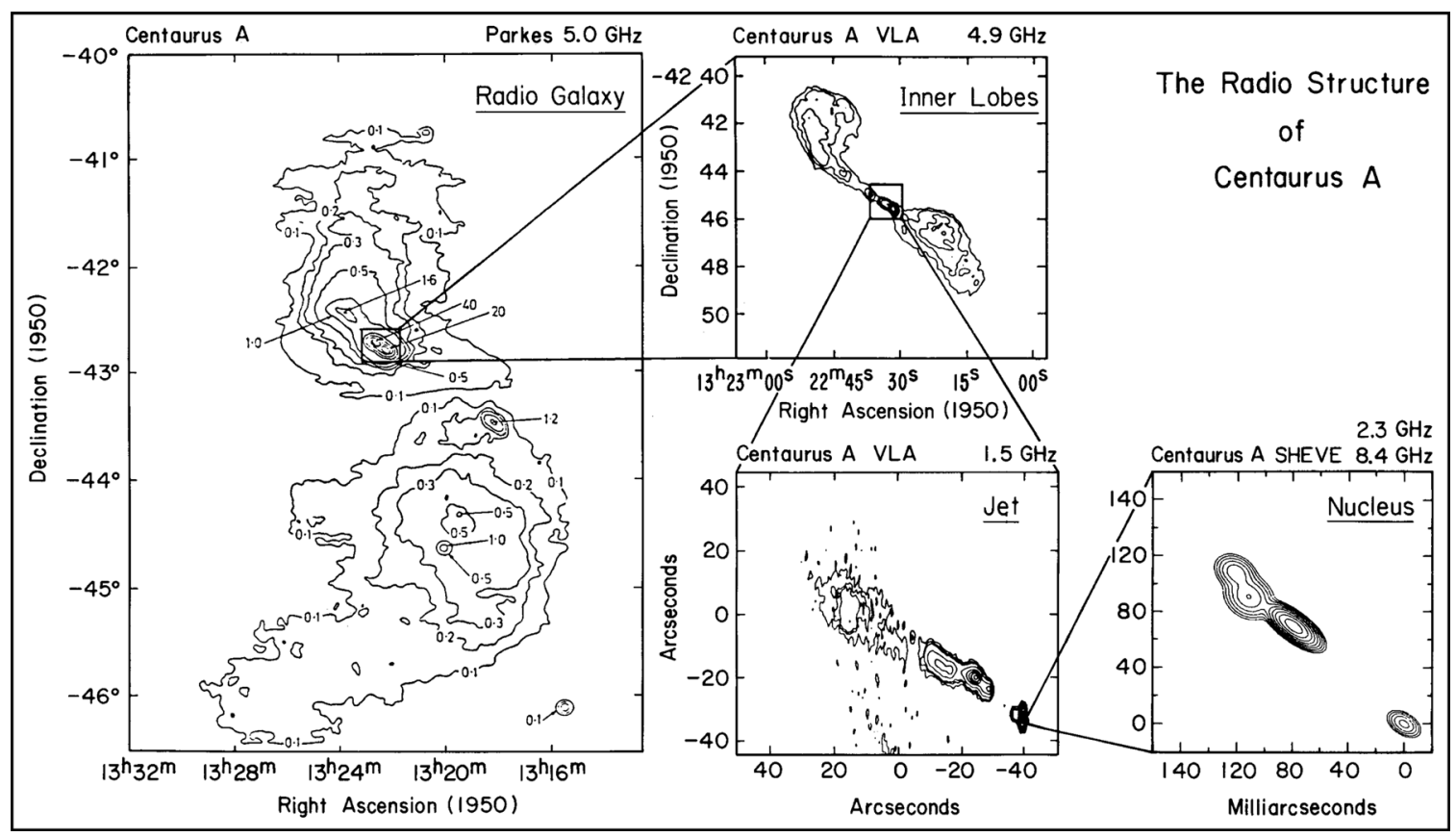

Figure 46 Montage of Cen A observations at scales varying by more than a factor of $10^{6}$. The bottom far right panel shows the fine structure of the Cen A nucleus (after Meier et al. 1988: 138).

observed sources, including plots of correlated flux densities and source maps which often showed milliarcsecond structural detail. In a second multi-authored paper (including Slee), Meier et al. (1989) provided a detailed account of the high-resolution structure of the Cen A nucleus at 2300 and $8400 \mathrm{MHz}$ (see Fig. 46), and in a third paper, involving Slee and 22 other authors, Tzioumis et al. (1989) discussed the compact galaxy 1934-638. Meanwhile, a fourth paper in this SHEVE series, on the Sgr A* Galactic Centre source, has already been discussed in Section 2.10 .

In the post-SHEVE era, Slee teamed with three other Australian astronomers in 1988 March to examine the angular structure of 34 early-type spiral and elliptical southern galaxies at $2290 \mathrm{MHz}$. For this purpose they used the Parkes-Tidbinbilla interferometer, with a baseline of $275 \mathrm{~km}$. The authors report:

"We found that $71 \%$ of the radio sources possess structure on a scale of tens of parsecs. We discuss the differences in total radio power, ellipticity, absolute optical magnitude, B-V colour, [NII] and IRAS emission between the detected and undetected galaxies. We found no convincing quantitative correlations between compact radio power and absolute optical magnitude, [NII] luminosity or radio power in the kiloparsec jets or outer lobes. Galaxies in the sample with dust lanes are no more likely to have detectable parsec-scale structure than non-dusty galaxies." (Slee, White, Reynolds and Caganoff, 1990: 361).

Slee's 1988 galaxy survey, above, drew heavily on data assembled by Sadler, and it is therefore appropriate that his next projects in this series were carried out in collaboration with her (and other ATNF colleagues). In the first of these projects Slee, Sadler, Reynolds and Ekers (1994)

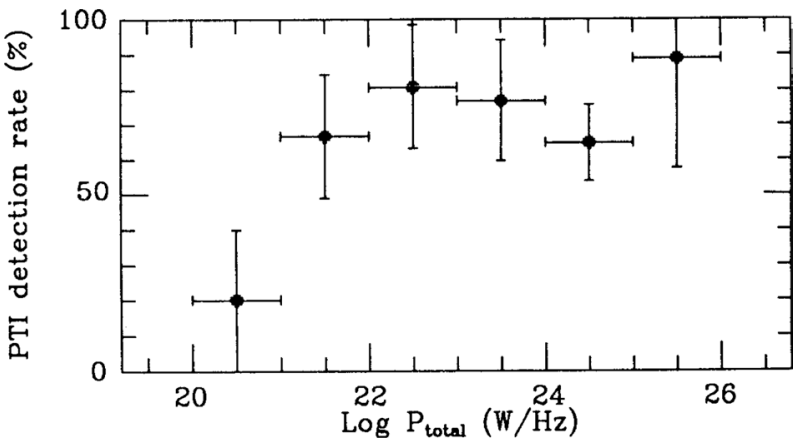

Figure 47 Detection rates of galactic cores with the ParkesTidbinbilla Interferometer plotted against total radio power (after Slee, Sadler, Reynolds and Ekers, 1994: 936).

studied two well defined samples of E and S0 galaxies using the Parkes-Tidbinbilla Interferometer at 2300 and $8400 \mathrm{MHz}$. The 'low-power sample' contained 69 galaxies with $m<14$, flux densities $>5 \mathrm{mJy}$ at $5000 \mathrm{MHz}$, and total radio powers of between $10^{20}$ and $10^{23} \mathrm{~W} \mathrm{~Hz}^{-1}$. The 'medium-power sample' contained 91 galaxies originally observed by Ekers (one of the co-authors), with $m<17$ and total radio powers of between $10^{22}$ and $10^{26} \mathrm{~W} \mathrm{~Hz}^{-1}$. A smaller and more heterogeneous sample of radioemitting Seyfert and spiral galaxies was also observed. The aim of this project was to see how core luminosities and spectra varied with total radio power and with other galaxy properties. The detection rate was found to be relatively independent of the total power emitted over a range of five orders of magnitude (see Fig. 47). A summary of the initial findings was presented at the Stromlo Symposium on the Physics of Active Galaxies (see Sadler, Slee, Reynolds and Ekers, 1994), and a full account was subsequently published in MNRAS. 
In this latter paper, Slee et al. reported that most of the galaxies detected with the Parkes-Tidbinbilla Interferometer were re-observed at $4700 \mathrm{MHz}$ with the Australia Telescope in order to check on flux variability and active core positions and to add another point to the core spectrum of each source. They found compact $(<0.03$ arc sec) radio-continuum cores in $\sim 70 \%$ of all galaxies, across the entire $10^{21}-10^{26} \mathrm{~W} \mathrm{~Hz}^{-1}$ range. These parsec-scale cores generally had a flat or rising spectrum between 2300 and $8400 \mathrm{MHz}$, with a median spectral index of +0.3 , and were similar in both size and spectral index to AGNs in radio galaxies and QSOs. No significant correlations occurred between core spectral index and core power, total power, core-to-lobe ratio and - where relevant data existed $\mathrm{X}$-ray luminosity and [NII] emission-line luminosity. Slee, Sadler, Reynolds and Ekers (1994: 945) suggested freefree absorption as the most likely emission mechanism, but did not rule out synchrotron self-absorption.

Slee et al. then used the Parkes-Tidbinbilla Interferometer in an attempt to answer the question: to what extent do Seyfert galaxies contain a 'central engine' similar to that found in active ellipticals and radio galaxies? Between 1988 and 1991 a search was made at 1700, 2300 and $8400 \mathrm{MHz}$ for compact $(<0.03$ arc sec) radio cores in 54 nearby galaxies of type SO/a or later, 22 of which were classified as 'active' and the remainder as 'normal'. Four of the 'active' galaxies were narrow-line X-ray galaxies, and the rest were Seyfert 1 or Seyfert 2 galaxies. Detection rates were illuminating, with $82 \%$ of Seyfert galaxies being detected at $2300 \mathrm{MHz}$ (as against just $22 \%$ for nonSeyfert galaxies), while at $8400 \mathrm{MHz}$ the figures were $18 \%$ and $15 \%$ respectively. The authors found that

"The radio emission from the inner kiloparsec of Seyfert galaxies is more diffuse and less centrally concentrated than in 'active' elliptical galaxies. Where parsec-scale radio cores are detected, they usually have steeper radio spectra than the cores in elliptical galaxies ... The difference in the median core spectral index between 2.3 and $8.4 \mathrm{GHz}(+0.3$ for elliptical galaxies, -1.0 for Seyferts), suggests that an active nucleus 'knows' on scales of 1-2 pc what kind of galaxy it inhabits." (Sadler, Slee, Reynolds and Roy, 1995: 1380).

Synchrotron emission was invoked as the likely generation mechanism for the radio cores in Seyfert galaxies.

\subsection{The Culgoora Source Survey}

When he returned to Sydney from the Royal Radar Establishment, long-baseline interferometry was not available in Australia at the time and so Slee decided to continue his study of radio galaxies using the Culgoora Radioheliograph (Fig. 48).

The Radioheliograph (Wild, 1967) was a novel array comprising 96 equatorially mounted $13.7-\mathrm{m}$ parabolic antennas spaced around the circumference of a circle $3 \mathrm{~km}$ in diameter. Completed in 1967, the Culgoora Radioheliograph was built to provide virtually instantaneous radio

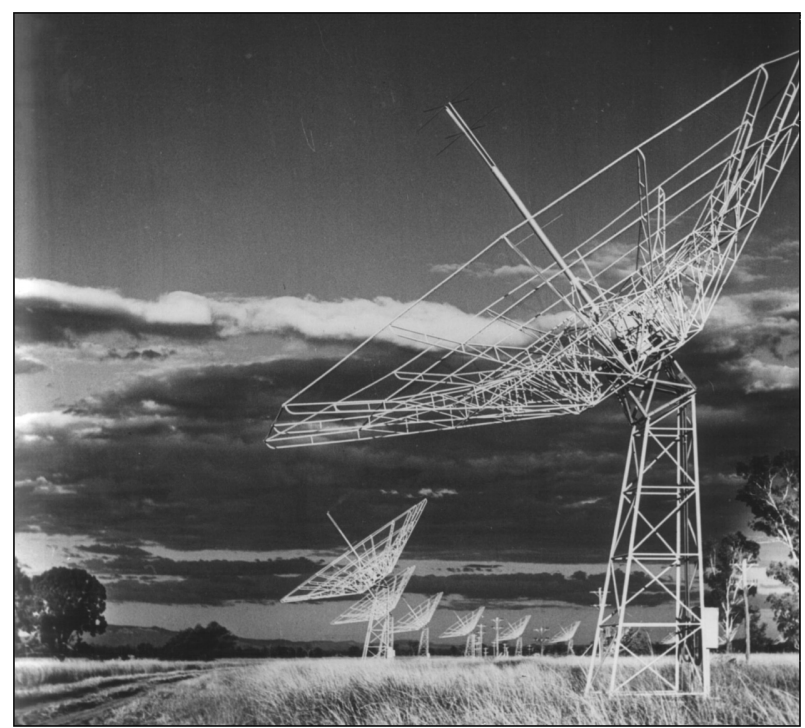

Figure 48 Panoramic view showing some of the 13.7-m antennas in the 96-element Culgoora Radioheliograph circular array (Photograph: ATNF Historic Photographic Archive, 8553-6).

images of the solar corona at $80 \mathrm{MHz}$, with emphasis on the dynamics of burst activity. Subsequently the array was up-graded so that it could also operate at 160 and 327.4 MHz (see Sheridan, Labrum and Payten, 1973). Slee was the first to see the potential of the array as a non-solar instrument, and over the years, until it was closed down in 1984, he was to make good use of it. As a non-solar array, the Radioheliograph could observe sources between $-48^{\circ}<\delta<+35^{\circ}$, and with as effective collecting area of $\sim 6000 \mathrm{~m}^{2}$ had an angular resolution of $\sim 3.7$ arc min at $80 \mathrm{MHz}$. This resolution was more than an order of magnitude better than that of the Mills Cross, the last radio telescope to be used for general southern survey work at metre wavelengths, and observations made in the interim at higher frequencies had highlighted the confusion that could be caused by the close juxtaposition of sources. The improved resolution would go a long way towards resolving this situation and, with the increased sensitivity, promised the detection of radio emission from QSOs, normal galaxies and distant radio galaxies.

So it was that in 1967 October (soon after the Radioheliograph began operating) Slee and Higgins commenced a survey of 548 of the stronger sources listed in Parkes catalogues with a view to determining their accurate positions, flux densities and angular sizes at $80 \mathrm{MHz}$. Also on their observing list were 183 sources listed in the MSH Fleurs catalogues but not detected at Parkes; 193 QSOs or suspected QSOs from the Parkes and $4 C$ catalogues; 30 normal galaxies observed by Cameron and Whiteoak at 408 and $5000 \mathrm{MHz}$, respectively; 25 supernova remnants recorded by Milne; and 20 pulsars. The observing program was completed in 1972 February, and a summary account of this first Culgoora source survey was subsequently published in the Astrophysical Supplement Series of the Australian Journal of Physics (Slee and Higgins, 1973). This long paper simply listed basic measurements for all 777 
observed sources, plus a separate table for the 222 undetected sources (with upper limits for their flux densities).

Observations for the second $80 \mathrm{MHz}$ Culgoora source survey were made during 1972 and 1973, the targets on this occasion being 1748 previously catalogued weak sources, most of which were drawn from the Parkes, Ohio and $4 C$ catalogues. In 1975, Slee and Higgins published 'Culgoora-2 List of Radio Source Measurements at $80 \mathrm{MHz}$ ' as another long Astrophysical Supplement. Base data on the 1291 observed sources were provided, together with a table listing the 457 undetected sources. Slee and Higgins (1975: 1) noted that these latter sources had low-frequency radio spectra similar to those of the identified radio galaxies rather than to those of the QSOs. They also compared the flux densities for 100 strong sources that were common to both surveys, noting that six of them had changed significantly during the three years between the two suites of observations.

Between 1972 July and 1976 July, Slee carried out the third Culgoora source survey. By this time the Radioheliograph had been upgraded to also operate at $160 \mathrm{MHz}$, and Slee decided to observe 1946 of the sources detected during his earlier surveys at this new frequency. He also observed a further 99 sources of special interest at 80 and $160 \mathrm{MHz}$. The third Culgoora list was published as another long (123-page) Astrophysical Supplement (Slee, 1977), and provided positions, flux densities and beam broadening values for all observed sources. Meanwhile, the improved resolution at $160 \mathrm{MHz}$ allowed Slee to include isophote plots for the 163 sources resolved by the 1.9 arc min beam.

Given Slee's personal involvement in the earlier Fleurs-Cambridge controversy over the $2 C$ catalogue, one interesting section of this third Culgoora sources paper was his comparison of $160 \mathrm{MHz}$ Culgoora-3 and $178 \mathrm{MHz}$ $4 C$ measurements. He began by reminding us that

"The Cambridge $4 C$ catalogue is an important source of positional and flux density information at metre wavelengths ... It is therefore important to check its accuracy ... There is no doubt that the Culgoora declination measurements are much more accurate, because the heliograph's beamwidth at $160 \mathrm{MHz}$ is a factor of 15 smaller than the $4 C$ declination beamwidth. In right ascension the interferometer fringe spacing of $7^{\prime} .5$ arc should result in a measurement accuracy comparable with that achieved by the heliograph's pencil beam at $1^{\prime} .9$ arc. The flux density measurements of the two sets of observations are tied to the same CKL scale and thus, after allowing for the small difference of frequency ... should permit identification of systematic differences; it is especially important to check the effects of the 4C interferometer fringes on the measured flux densities of slightly extended sources." (Slee, 1977: 5; my italics).

A total of $10064 C$ sources was included in the Culgoora-3 list, and while there was no significant difference between R.A. positions in the two catalogues, the flux density
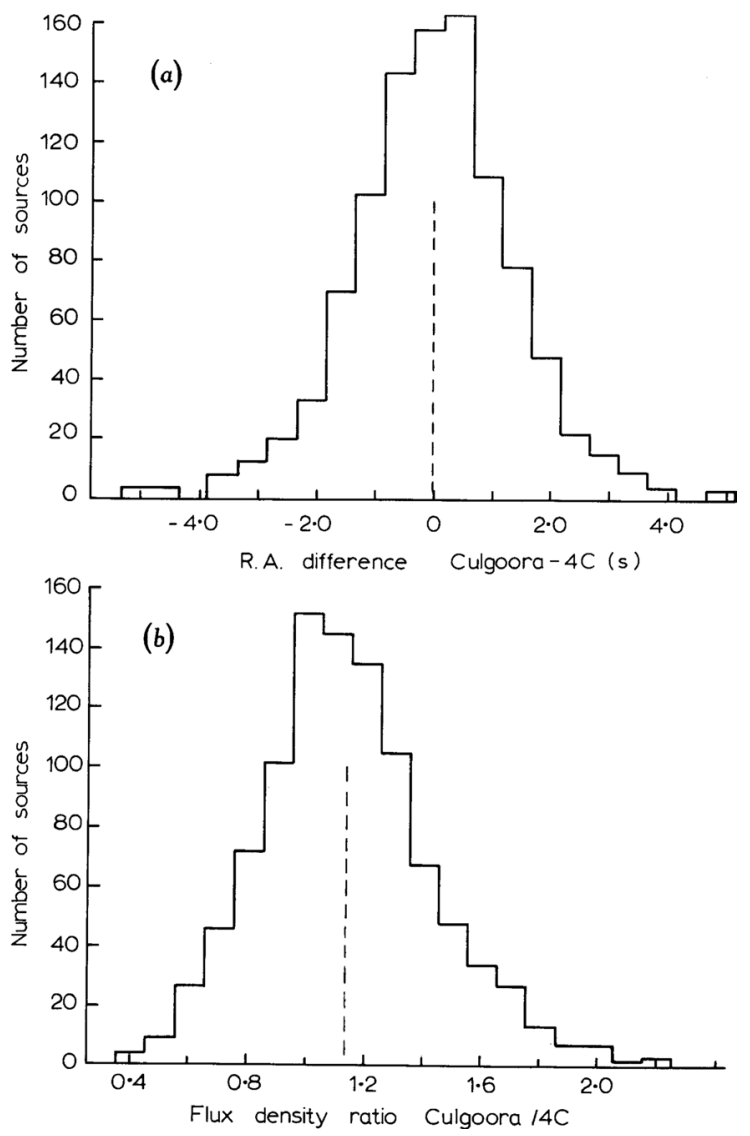

Figure 49 Histograms for 1006 sources common to the $4 C$ and Culgoora-3 lists, showing distributions of R.A. (a) and flux density (b) (after Slee, 1977: 9).

measurements were significantly different with Culgoora3 flux densities $\sim 14 \%$ higher than their $4 C$ counterparts (see Fig. 49).

As the three Culgoora surveys progressed, Huchtmeier and Slee (1976) had time to conduct a survey of compact galaxies at 2700 and $5000 \mathrm{MHz}$ with the Parkes Radio Telescope. Slee also found himself in an ideal position to research several interesting Culgoora projects in depth. For example, using data on the 30 normal galaxies observed at $80 \mathrm{MHz}$ in 1970 and 1971 he was able to assemble a table listing their flux densities, differences in their radio and optical positions, and their optical and radio angular sizes. Eight galaxies were resolved at $80 \mathrm{MHz}$, but only one of these was large enough for brightness contours to be plotted (see Slee, 1972a).

Slee also used data on the aforementioned galaxies to construct a spectrum for each galaxy by combining his own results with other people's published and unpublished data at 85.5, 408, 1410, 2650 and $5000 \mathrm{MHz}$. An interesting statistic was that about one-third of all galaxies showed spectral turnovers, with flux densities at $80 \mathrm{MHz}$ well below the extrapolated high-frequency spectra. Slee (1972b: 161) noted that this was a feature of a wide variety of morphological types (including elliptical galaxies and ordinary and barred spirals), and that further research was required. 

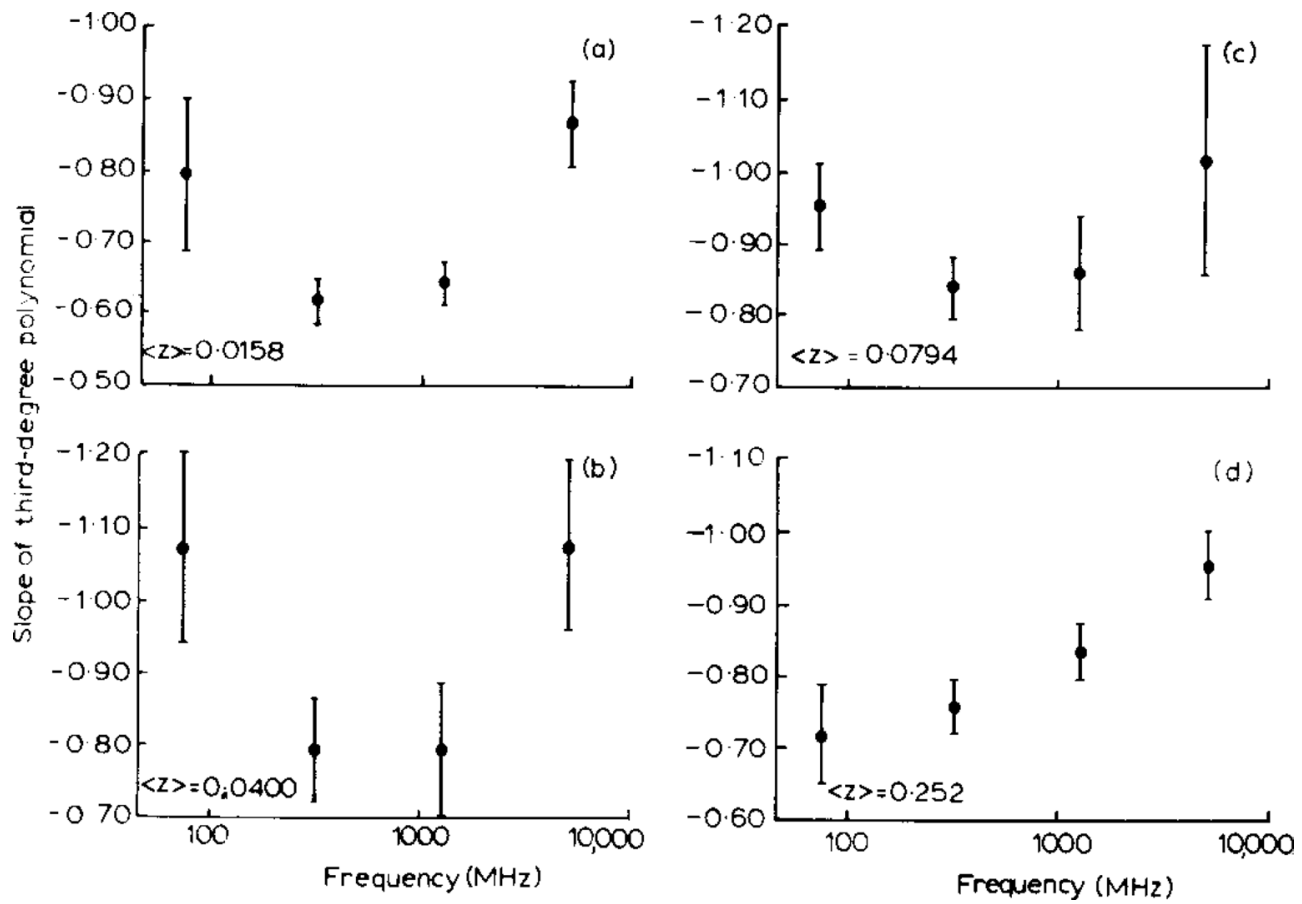

Figure 50 Average slopes of best-fitting third-degree polynomials for sources with widely-differing redshifts (after Slee, 1982: 37).

Spinning off from the Culgoora-3 survey and the $160 \mathrm{MHz}$ observations was a paper in which Slee and Sheridan (1975) reproduced high-resolution isophote maps of Cen A and Pic A and discussed these two galaxies in the light of results provided by other workers (many of them Radiophysics colleagues) at different frequencies.

Also emerging from the Culgoora-3 survey was a major paper on the spectra of $\sim 2000$ radio sources, which at the time was the largest analysis of this kind ever undertaken. In this study, Slee and his two Radiophysics collaborators combined Culgoora data with flux densities reported at many different wavelengths by other observers, and by including the 80 and $160 \mathrm{MHz}$ Culgoora values in many instances they were able to extend the frequency base downwards by a factor of five. For each source the accumulated flux values were fitted with first, second and third degree polynomials in order to determine the spectral index. The aim then was to identify spectral features that were common to sources associated with the same types of optical objects. Slee, Siegman and Mulhall (1982: 292) listed the major conclusions of their study: (1) QSOs differed from radio galaxies in that they had a distribution of linear spectral indices with a pronounced tail towards the low values of the spectral index; (2) D and cD galaxies had significantly steeper spectra than other classes of radio galaxies; (3) unidentified sources had a mean spectral index that was significantly higher than the mean spectral index for all identified radio galaxies; (4) there was significant second-degree curvature in all identification classes; radio galaxies and unidentified sources had spectra which steepened with increasing frequency, while QSO spectra became flatter; (5) there were minima in the spectral slopes of radio galaxies (but not QSOs) at $\sim 500 \mathrm{MHz}$, but these were not obvious in the spectra of unidentified sources; and
(6) radio luminosities of Culgoora- 3 sources with measured redshifts ranged from $1.5 \times 10^{30} \mathrm{~W} \mathrm{sr}^{-1}$ for QSOs down through N, D, E and db galaxies to $4.7 \times 10^{25} \mathrm{~W} \mathrm{sr}^{-1}$ for 'normal' spirals.

As a follow-up to the above paper, Slee prepared another on a spectral feature which he though may have evolutionary significance. When only those Culgoora-3 sources with a reasonable chance of optical identification (i.e. $|b|>10^{\circ}$ ) were considered, there was some indication that relationships existed between spectral index and redshift, spectral index and radio power, and also spectral index and linear dimension. If all three types of relationship could be explained in terms of a direct dependence of spectral index on redshift then this had obvious evolutionary implications, but other explanations were also possible and Slee (1981) noted that this issue could not be resolved with existing datasets.

This thought-provoking paper was immediately followed by another which examined a further concluding point in the Slee, Siegman and Mulhall paper above, namely that in many cases there were minima in the spectral slopes of identified radio galaxies at $\sim 500 \mathrm{MHz}$, but these were not a feature of unidentified sources. In order to examine the possible redshift dependence on spectral shape, identified radio galaxies were subdivided into four groups based on increasing redshift, and when spectral slope was plotted against frequency the first three plots showed the characteristic deep minimum but this was absent in the fourth plot $(<z>=0.252)$. Instead, this anomalous plot was reminiscent of that obtained for unidentified sources (see Fig. 50). After examining various possible interpretations, Slee (1982: 38) concluded: "It is difficult to propose an explicit evolutionary model that could reproduce the observed spectral features; the 


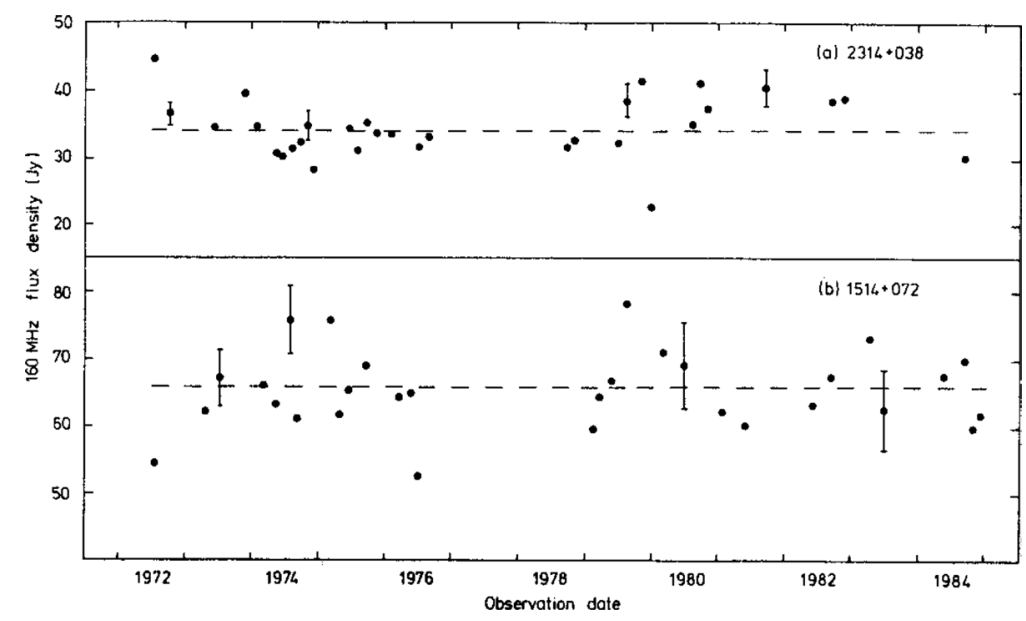

Figure 51 Plots of one-monthly averaged $160 \mathrm{MHz}$ flux densities for sources $2314+038$ (a) and 1514+072 (b), between 1972 and 1984 (after Slee and Siegman, 1988: 1316).

theory of source evolution is still in rather a primitive state and treats only the simplest source geometries (Melrose, 1980) incorporating only one or two of the several possible evolutionary processes."

After completion of the third Culgoora source survey in 1976, Slee continued to make intermittent 80 and $160 \mathrm{MHz}$ observations with the Radioheliograph through to 1984 , mainly in order to accumulate data for several new stand-alone projects. However, in one notable instance he observed additional sources and added them to the earlier Culgoora survey data in order to draw on an enlarged databank for a detailed statistical analysis of intensity variations in extragalactic sources. The 412 sources were selected at random and included QSOs and a full range of galaxy types, and the analysis showed that: (1) $47 \%$ of all sources displayed variability at 80 and/or $160 \mathrm{MHz}$ on time scales of months or years (see Fig. 51); (2) QSOs, radio galaxies and unidentified galaxies all showed comparable levels of variability; (3) sources with flatter spectra were more variable than those with steeper spectra; (4) there was a suggestion that sources at low Galactic latitudes varied more than those at higher latitudes; (5) sources with month-to-month variability showed even more variability on a year-to-year basis; and (6) variability levels at 80 and $160 \mathrm{MHz}$ were well correlated for sources displaying variability at both frequencies. Slee and Siegman (1988: 1313) felt that their results were best explained in terms of the focussing and occulting effects of compact interstellar ionised structures that had been recently identified at microwave frequencies.

One of these new post-1976 Radioheliograph projects was a radio survey of extragalactic X-ray sources. Earlier attempts to correlate $\mathrm{X}$-ray and radio sources had been fraught with difficulties, and in a bid to improve positional and spectral data Slee and an Australian collaborator carried out a systematic search for $80 \mathrm{MHz}$ radio emission at the locations of all $47 \mathrm{X}$-ray sources recorded by Ariel V. When radio sources were detected, these were then re-observed at $160 \mathrm{MHz}$. Slee and Quinn (1979: 340) listed the results of their study, including: (1) 40 radio sources were detected near 22 of the X-ray sources, and upper limits to the radio flux were placed on the remaining X-ray sources; (2) 29 radio sources were detected in 17 of the 27 clusters of galaxies associated with Ariel V sources; (3) X-ray and $80 \mathrm{MHz}$ sources occurred more frequently in richer clusters of galaxies and in Bautz-Morgan Class I clusters; (4) low-frequency spectral indices of radio sources in clusters with $\mathrm{X}$-ray sources were generally much higher than those in the general radio source population; (5) there was no obvious relationship between the low-frequency radio spectral index and X-ray luminosity, cluster richness or Bautz-Morgan class, but X-ray and radio luminosities were related; (6) some X-ray sources were associated with individual radio galaxies; and (7) in clusters of galaxies, extended X-ray sources were associated with gas in the inter-galaxy medium. Through this project, Slee would embark on a whole new field of astronomy - clusters of galaxies - as outlined Section 2.14 below.

Another of these post-1976 Radioheliograph projects involved Slee in a collaboration with three Australian colleagues in order to map the inner lobes of Cen A. The Radioheliograph observations were made at $327 \mathrm{MHz}$, with a beamwidth of $54 \mathrm{arcsec}$, and the resulting isophote plot was compared and contrasted with maps provided at 843 and $1415 \mathrm{MHz}$ by the Molonglo and Fleurs Synthesis Telescopes respectively (see Fig. 52). Although a VLA map published in 1981 offered more fine detail in the central region, the Australian observations provided flux densities and spectral data for the two inner lobes of this galaxy. Finally, Slee, Sheridan, Dulk and Little (1983) concluded by discussing possible mechanisms that could explain the galaxy's radio and X-ray emission.

One last post-survey stand-alone Radioheliograph project deserves to be mentioned, and this was Slee's on-going observation of the QSO 1055+018. This source was first recorded in 1970, and was also observed in 1975 during the third Culgoora survey. Then between 1979 January and 1983 July it was observed on five different 

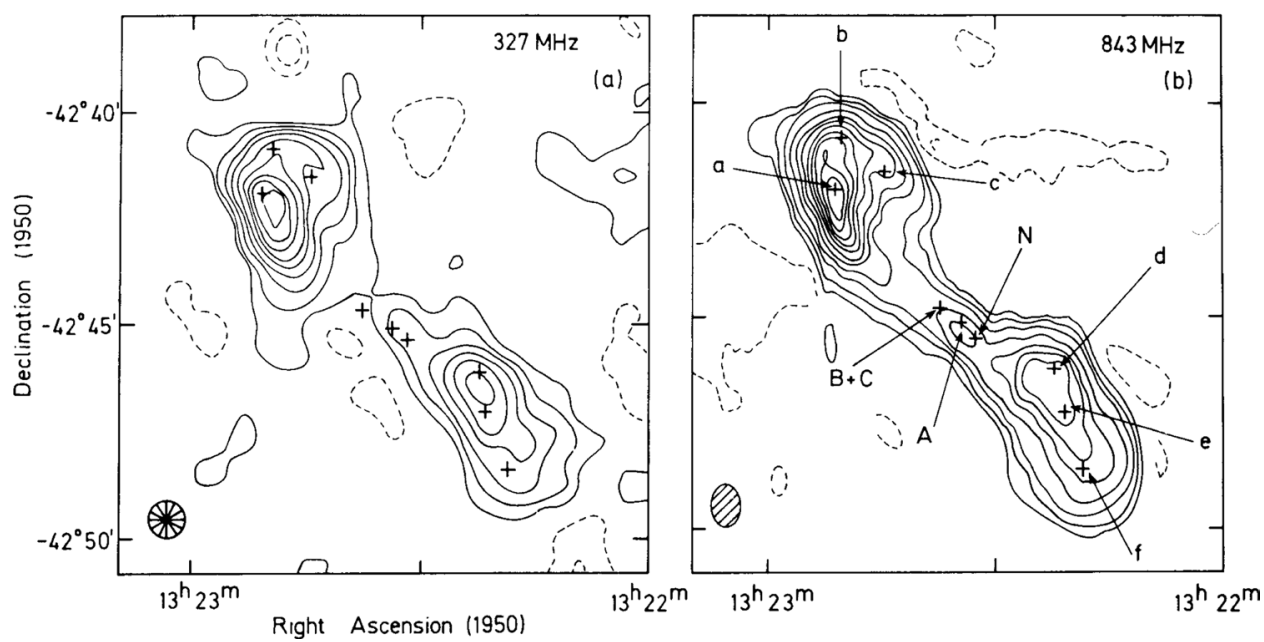

Figure 52 Maps of the inner lobes of Cen A based on Culgoora (a) and Molonglo (b) observations. The crosses and lettering in (b) refer to positions of brightness peaks on the 1415 MHz VLA map (after Slee, Sheridan, Dulk and Little, 1983: 248).

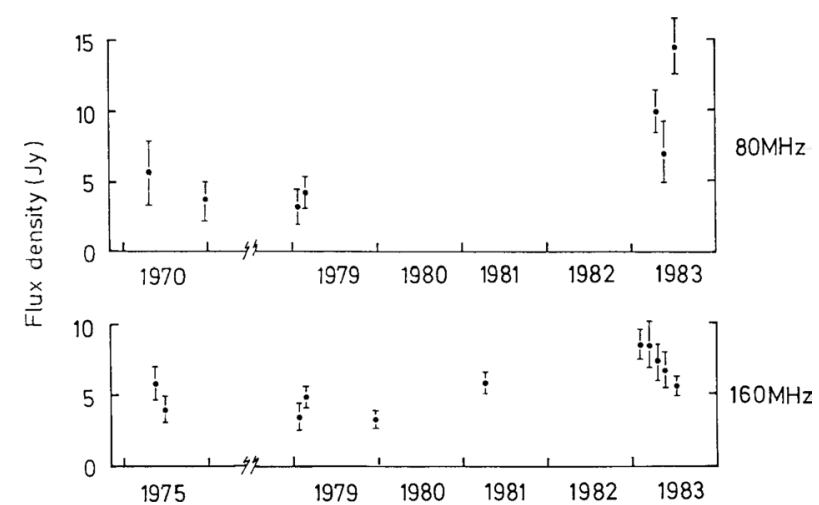

Figure 53 Flux density measurements at 80 and $160 \mathrm{MHz}$ for the QSO 1055+018, showing the 1983 'outburst' (after Slee, 1984: 217).

dates at $80 \mathrm{MHz}$ and nine dates at $160 \mathrm{MHz}$, and when the readings were plotted the flux densities were found to have tripled and doubled respectively during this period. Slee referred to this as a metre-wave 'outburst' or 'flare' (see Fig. 53), and noted that no corresponding enhancement was observed at decimetre or centimetre wavelengths. After discussing various competing generation mechanisms, he pointed out that the variability exhibited by $1055+018$ was by no means unique in that this was a feature of $\sim 10 \%$ of the flat-spectrum sources he had observed (Slee, 1984: 225).

By the time Frater was appointed Chief of Radiophysics in 1981 the Division was undergoing a strategic realignment of pure and applied research priorities and it was merely a matter of time before the demise of the Radioheliograph. An ongoing decline in solar activity and the expense of a costly overhaul to bring the array up to standard for further meaningful solar work clinched the matter and in 1984 it was closed down.

This brought to an end 17 years of invaluable solar and non-solar work (see Haynes et al., 1996: 285). This also brought Slee's formal involvement with this novel array to an end, but it was only in 1995 that he published "... a final updated and recalibrated list of sources that were observed with the Culgoora circular array in the interval 1970-1984." (Slee, 1995: 143) in order to bring the whole Culgoora source survey work to a formal close. This paper contained all sources listed in the Culgoora-1, 2 and 3 papers plus additional sources detected in the course of a survey of various Abell clusters of galaxies, and for further ease of access all data were made available in electronic form. In 1995, Slee and two Russian colleagues also investigated the asymmetrical distribution in the centreanticentre region of variable radio sources detected during the Culgoora surveys (Shapirovskaya, Slee and Tsarevsky, 1995).

\subsection{Clusters of Galaxies}

Although Slee's involvement with clusters of galaxies was very much a child of the 1980s and 1990s, with the Culgoora Radioheliograph and later the VLA, his first paper in this field (Komesaroff, Slee and Higgins, 1968) actually dated to the early Parkes era. In a collaboration with two Radiophysics colleagues, he used the 64-m antenna and the MK II 11-cm receiver to search for radio emission from 38 Abell clusters of galaxies. Altogether, 24 sources with flux densities $>0.3$ Jy were detected in 13 of these clusters, but no details were provided in their preliminary paper.

Slee's next involvement with clusters of galaxies came more than ten years later when three Russian astronomers, impressed by the 1979 paper by Slee and Quinn on radio emission from Ariel V X-ray sources, included him in a paper on their observations of $24 \mathrm{X}$-ray sources at 102.5 MHz using the BSA FIAN radio telescope. All of these sources were associated with clusters of galaxies, and radio emission was detected from all but three of them. After measuring radio flux densities and calculating spectral indices for these sources, the authors found weak evidence for a correlation between this latter parameter and X-ray luminosity. They also found that five of the fifteen strong X-ray emitting clusters appeared to contain large-diameter radio sources with linear dimensions 
approaching those of the whole cluster, while the remainder were characterised by radio sources with much smaller linear dimensions - only of a few hundred kpc at most (Dagkesamansky, Gubanov, Kuzmin and Slee, 1982: 986).

In his first major independent survey of radio emission from clusters of galaxies, between 1978 and 1981 Slee and two Australian colleagues examined 353 Abell clusters using the Culgoora Radioheliograph at 80 and $160 \mathrm{MHz}$ and 80 Abell clusters using the Parkes Radio Telescope at $2700 \mathrm{MHz}$. For general reference purposes, Slee and Siegman (1983) published a master list of source properties, including positions, $160 \mathrm{MHz}$ flux densities, spectral indices, and information on the associated clusters of galaxies.

In an initial analysis of the spectra of 147 sources associated with these clusters, Slee et al. found that spectral index decreases systematically with distance from the cluster centre and that "... sources in clusters have significantly steeper spectra than the field radio galaxies ... [due to] the confining influence of a hot, relatively dense electron gas. In fact, the steepening of the radio spectrum provides a more sensitive method of detecting this gas than does the use of existing X-ray telescopes ..." (Slee, Wilson and Siegman, 1982: 433-434).

These three authors then published a statistical survey of the general properties of 148 radio sources supposedly associated with Abell clusters of galaxies compared to 127 field galaxies with the same range of redshifts drawn from the Culgoora-3 survey. For sources associated with Abell clusters they found (1) sources were highly concentrated towards the cluster centre; (2) the probability of finding a source in a cluster was not related to cluster richness; (3) sources were relatively more common in Bautz-Morgan Class I clusters; (4) spectral index was not related to cluster richness, but increased towards cluster centres and was highest for Bautz-Morgan Class I clusters; (5) radio power at $160 \mathrm{MHz}$ was highest for sources within 0.10 Abell radius of the cluster centre, but was not related to cluster richness or optical morphology. Upon comparing the cluster sources with those associated with non-cluster galaxies they reported that (1) cluster galaxy sources had relatively higher spectral indices; (2) although second- and third-degree curvature was a feature of both cluster and field galaxy sources, it was more pronounced with cluster galaxies; (3) cluster galaxy sources were not particularly strong emitters, the median radio power of field galaxy sources at $160 \mathrm{MHz}$ was about four-fold greater; (4) field galaxy sources had median linear dimensions $\sim 130 \mathrm{kpc}$ larger than cluster galaxy sources; and (5) the luminosity functions of both types of sources had a power-law form and possessed very similar power-law exponents (Slee, Wilson and Siegman, 1983).

In order to progress his 'clusters' research, Slee then decided to make use of the VLA, but his first paper in a new series, co-authored with an Australian colleague, also drew on Culgoora Radioheliograph observations. Slee and Reynolds noticed that among the sources associated with clusters of galaxies observed at Culgoora

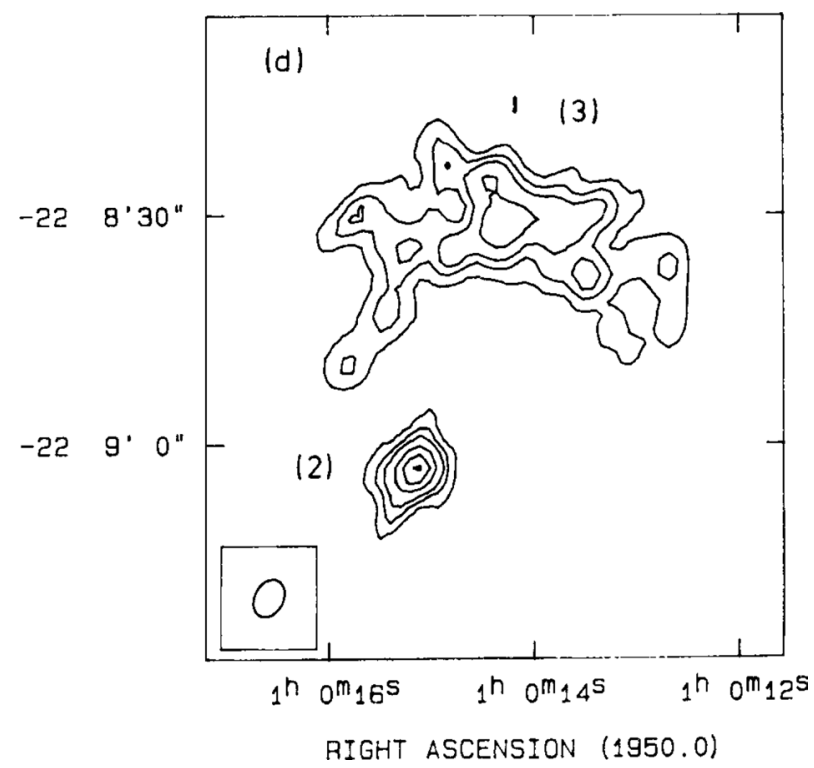

Figure $541465 \mathrm{MHz}$ contour map of a steep-spectrum source (3) that cannot easily be associated with a galaxy in the Abell 133 cluster (after Slee and Reynolds, 1984: 521).

and with the $408 \mathrm{MHz}$ Molonglo Synthesis Telescope (MOST) were a number with unusually steep spectral indices (i.e. $\alpha<-1.5$ ). In order to learn more about these spectral indices and compare them with radio, optical and X-ray morphologies they selected six southern sources, and in 1981 August and 1983 May-June these were observed at 1465 and $4886 \mathrm{MHz}$ using the VLA with beamwidths of between $\sim 4$ and 12 arc sec. All six sources were also observed with MOST at $843 \mathrm{MHz}$ between 1981 September and 1982 December, with a beamwidth of $40 \mathrm{arc} \mathrm{sec}$. On the basis of the isophote plots and calculated spectral indices, Slee and Reynolds (1984) listed the following conclusions: (1) steep-spectrum sources near cluster centres were found to be complex and contained different components with differing spectral indices; (2) four of the steep-spectrum sources did not contain a cluster galaxy that could easily account for the radio emission (e.g. see Fig. 54); (3) two of the steep-spectrum sources were possibly head-tail sources; and (4) four of the steep-spectrum sources were in clusters with high $\mathrm{X}$-ray emission, while the other two were in more distant clusters that had not been detected in X-ray surveys to that time. Slee and Reynolds (1984: 528) believed that several components of these steep-spectrum sources present, particularly, in the VLA maps, may have been ejecta relics, and they suggested two possible explanations for their formation: "(i) the source is a fossil relic of a radio galaxy (perhaps a tail) that has been swept up by the massive central cD or giant elliptical; (ii) the steep-spectrum component that we now see is the aged remnant of a plasmoid that was ejected from the bright central galaxy at a speed close to or somewhat higher than the sound speed in the intra-cluster gas."

This initial use of the VLA led Slee to initiate the "VLA Survey of Rich Clusters of Galaxies', with a view to examining their spectral indices, morphologies, distributions 


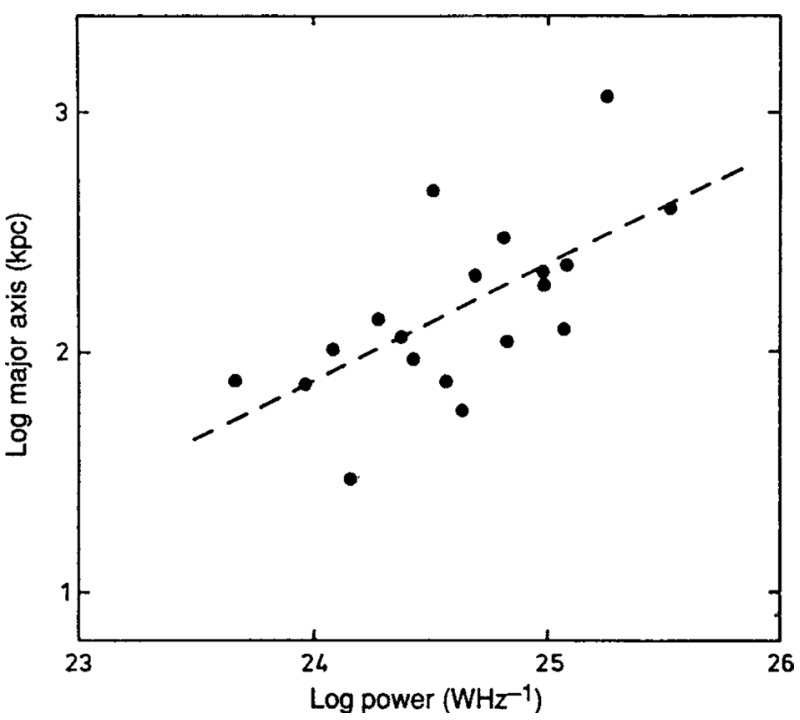

Figure 55 Plot of largest linear size vs radio power for 20 cluster doubles observed at $1500 \mathrm{MHz}$ with the VLA, and the least-squares line of best fit (after Slee, Perley and Siegman, 1989: 727).

with respect to cluster centres, polarisation, radio power, and its relation to X-ray luminosity. This project commenced in 1981, and between this date and 1985 Slee and his Australian and international collaborators carried out a radio survey at 1500 and $4900 \mathrm{MHz}$ of 58 cluster fields centred on sources with spectral indices $\alpha \leq-0.90$ (defined by $S \propto \nu^{\alpha}$ ). The initial paper reporting on this project included data on all 940 sources detected with $S_{1.5} \geq 1 \mathrm{mJy}$, together with whole-field source maps at $1500 \mathrm{MHz}$ and spectral indices for all sources $<5$ arc min from the field centres. A statistical analysis of the assembled data and optical properties of the clusters revealed that source area and largest linear size both increased with radio power (e.g. see Fig. 55); total cluster radio power increased with cluster richness; the spectral index was highest in those clusters with dominant central galaxies; and in resolved double sources, the component with the higher flux density also had the higher spectral index (Slee, Perley and Siegman, 1989).

The second paper in the VLA Clusters series drew on data prepared for paper 1, and examined the morphology, polarisation, spectral index and optical identification of sources with integrated flux densities of $S_{1.5} \geq 20 \mathrm{mJy}$. In addition, many of these radio sources were observed at $8400 \mathrm{MHz}$ (in 1989 April) with the Parkes-Tidbinbilla Interferometer in order to examine their cores. Slee et al. noted that in many instances these sources were the most powerful in the associated clusters. Their main conclusions included: (1) 79 of the 251 radio galaxies detected were resolved into double or triple sources; (2) $49 \%$ of all radio galaxies were identified with optical counterparts; (3) the radio power distributions of sources with and without optical identifications were almost identical; (4) radio cores were detected for just 10 sources, and upper limits of $\sim 6 \mathrm{mJy}$ were placed on the remainder; (5) sources with and without optical identifications had significantly different median sizes, at 74 and $34 \mathrm{kpc}$ respectively; (6) spectral

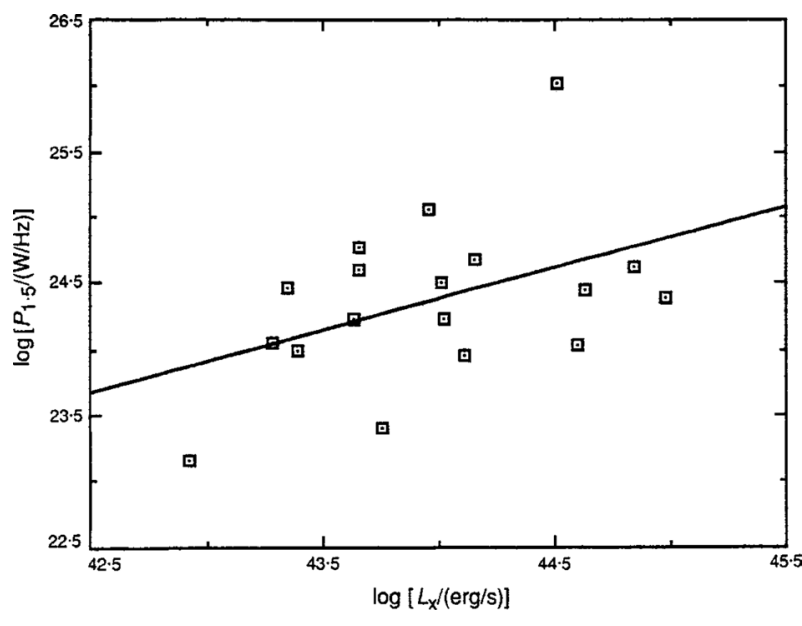

Figure 56 Plot of $1500 \mathrm{MHz}$ VLA radio flux vs X-ray luminosity for 18 cluster fields with centrally-located X-ray sources, showing the least-squares line of best fit (after Slee, Roy and Savage, 1994: 239).

indices for sources with and without identifications were virtually identical, but for sources close to cluster centres and those at larger angular distances they were significantly different; (7) linear polarisation was detected in 35 of the 59 sources observed at $4900 \mathrm{MHz}$ (with values ranging from $5 \%$ to $67 \%$ ), and for 19 of the 49 sources observed at $1500 \mathrm{MHz}$ (with values from 5\% to $37 \%$ ); and (8) there was a significant correlation between $1500 \mathrm{MHz}$ radio flux and X-ray luminosity, as shown in Fig. 56 (Slee, Roy and Savage, 1994).

Having dealt with the strongest sources, in their third paper in this series, Slee et al. turned their attention to the 737 weakest sources in their VLA survey $(1<$ $S_{1.5}<20 \mathrm{mJy}$ ). On this occasion, their main interest was in the morphology and optical identification of sources, but on the assumption that relatively fewer sources would be cluster members, and this was born out by the fact that only 174 sources $(24 \%)$ were identified with galaxies. Since most observations were made at $1500 \mathrm{MHz}$ the majority of isophote plots lacked detail, but 62 sources were recorded with double structure; only 18 of which were associated with known galaxies. There were also six triple or quadruple sources, but only two of these were identified with galaxies. Other parameters that were tabulated - but not subjected to detailed statistical analysis - were angular size, spectral index and, for those sources with published redshifts, emitting power and linear size (see Slee, Roy and Andernach, 1996).

The fourth and final paper in the VLA Clusters series had the challenging subtitle, 'An Analysis of Results', and it included the following conclusions: (1) the fraction of sources identified with galaxies rose from $\sim 28 \%$ at lower flux levels to $\sim 80 \%$ at high flux-densities; (2) $\sim 7 \%$ of sources were associated with star-like objects, about twice the chance expectation, suggesting that half of them are QSOs; (3) two different samples of identified radio cluster galaxies were identified, drawn from clusters surveyed completely out to 0.24 and 0.51 Abell 
radii, respectively; (4) sources in these two samples were concentrated towards the centres of their clusters, with surface density varying as $\left(r / R_{\mathrm{A}}\right)^{-1.6}$ (Fig. 57); (5) the lobes of cluster doubles had spectra that steepened with radio power and with surface brightness (the opposite of double-lobed QSOs); (6) for both samples of identified radio cluster galaxies there was a significant correlation between galaxy absolute red magnitude and $r / R_{\mathrm{A}}$; (7) where polarisation was detected in radio lobes at both 1500 and $4900 \mathrm{MHz}$, depolarisation was caused by tangled magnetic fields (see Fig. 58), and where it was only detected at $4900 \mathrm{MHz}$ by a combination of tangled fields and differential Faraday rotation; (8) in double radio sources for which polarisation was detected at both 1500 and $4900 \mathrm{MHz}$ the favoured magnetic field direction was

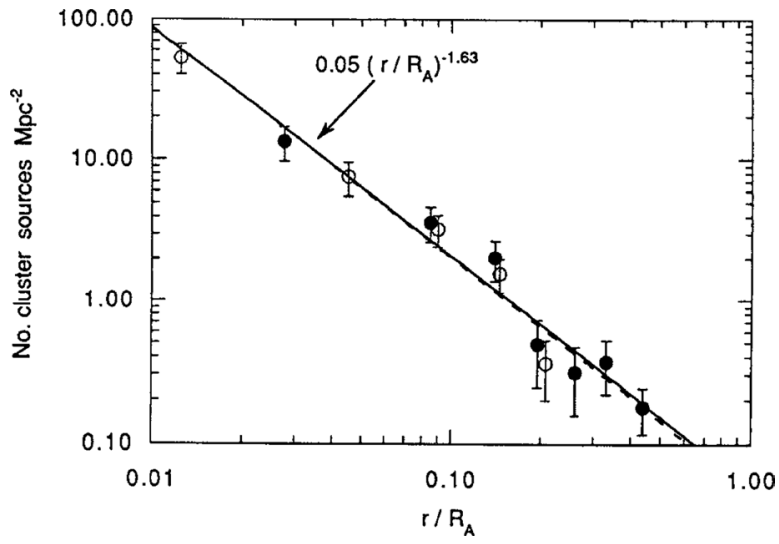

Figure 57 Radial variation in the surface density of cluster sources, with the power-law fit (after Slee, Roy and Andernach, 1998: 980). parallel to the axis, and presumably the unresolved jets; (9) lifetimes of cluster radio galaxies depended mainly on the magnetic field strength in their lobes, and any de-acceleration processes operating; (10) lifetimes of radio cluster galaxies varied depending upon polarisation levels and field characteristics, but all were $<1.2 \times 10^{7} \mathrm{yr}$; (11) it could be shown that relativistic plasma ejected from a single acceleration site in a host galaxy at an average speed of $\geq 3000 \mathrm{~km} \mathrm{~s}^{-1}$ could account for the ages mentioned in (10) above; (12) confinement of lobes could easily be explained by the static pressure exerted by the inter-cluster gas, assisted by ram pressure generated by the movement of the galaxy through the medium; (13) cluster X-ray luminosity was correlated with both total radio power and the red absolute magnitude of the cluster; and (14) since core power in cluster radio galaxies was similar to that in non-cluster radio galaxies it seemed that the high density of hot gas near the centre of a cluster did not provide an additional fuel supply for the nuclear engine (Slee, Roy and Andernach, 1998).

Having drawn attention to 'relics', or arc-like steepspectrum components of cluster radio galaxies, in several different papers (e.g. see Andernach, Gubanov and Slee, 1998), the search was now on to document further unusual examples. In 1997 January, Slee and Roy carried out 1425 MHz VLA observations of Abell 4038_9, an extreme example of a relic that was apparently not associated with an optical galaxy. With access to 3 arc sec resolution, they were able to generate a detailed contour map showing an extended source $56 \mathrm{kpc}$ in length situated a mean distance of $42 \mathrm{kpc}$ from the centroid of the Abell 4038 cluster (see Fig. 59). By imaging the two L-band channels at 1385 and (c) A2091_6a/b $4.9 \mathrm{GHz}$ Class I

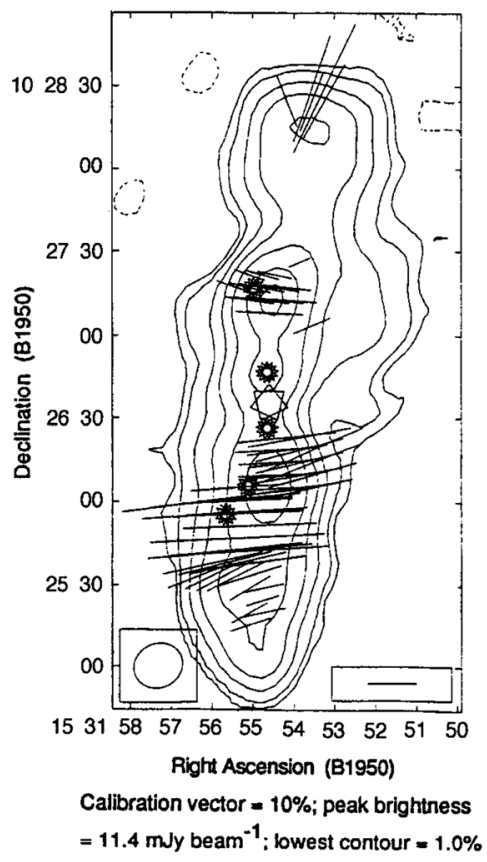

(d) A2091_6a/b $1.5 \mathrm{GHz}$ Class I

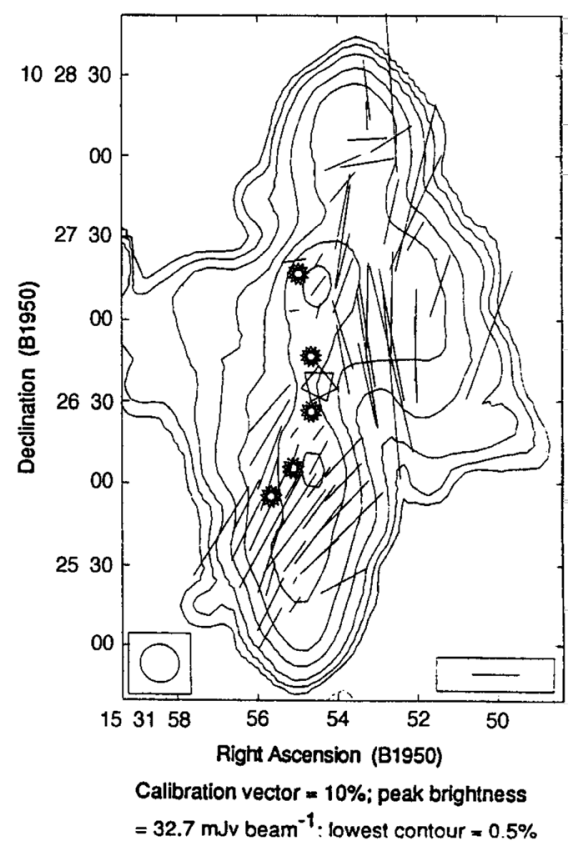

Figure 58 Maps of A2091_6a/b at $4900 \mathrm{MHz}$ (left) and $1500 \mathrm{MHz}$ (right), with superimposed electric vectors. Optical galaxy positions are shown by the stars (after Slee, Roy and Andernach, 1998: 988). 
$1465 \mathrm{MHz}$ separately, they were able to measure the spectral index of $\alpha=-2.2 \pm 0.1$ (Fig. 60), consistent with the value derived previously using data from an 18:1 range of frequencies. The shape of the southern extension of the source resembled a narrow-angle-tail source, but the absence of an obvious host galaxy made it unlikely that the source was a working radio galaxy. Instead, Slee and Roy (1998: L86) suggested that it was probably the remains of an FR II radio galaxy that was originally energised by a particularly bright elliptical galaxy $18 \mathrm{kpc}$ away. They assigned an age of $6 \times 10^{6} \mathrm{yr}$ to the source.

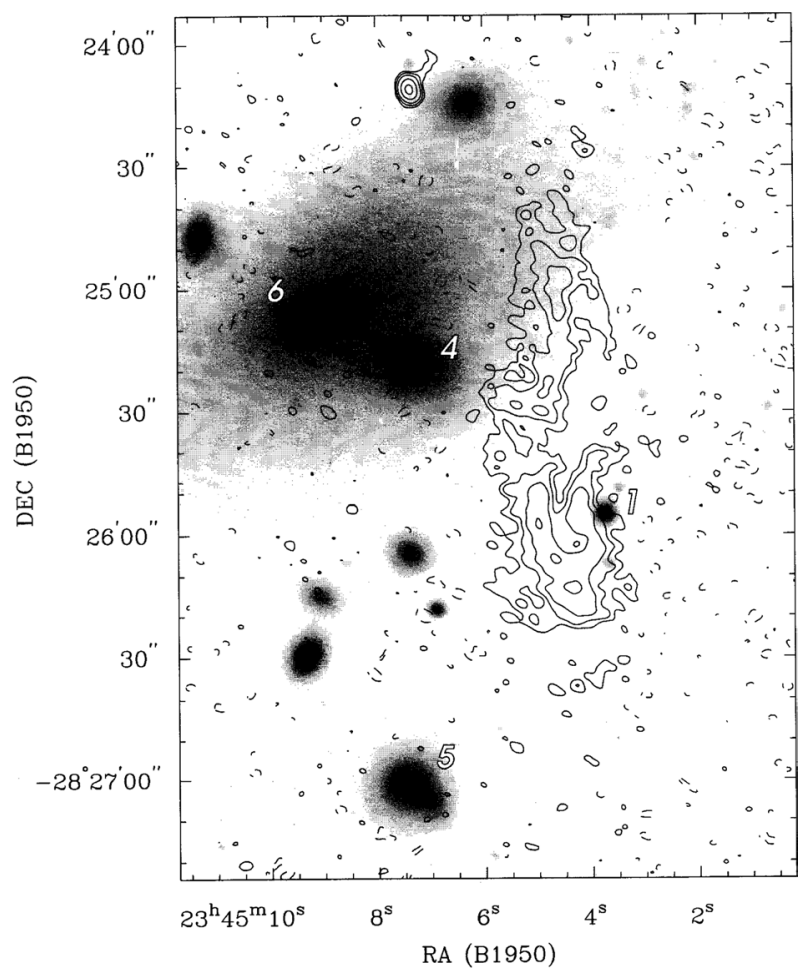

Figure 59 VLA $1425 \mathrm{MHz}$ isophote map of the steep-spectrum relic A4039_9. The large extremely bright galaxy to the northeast of the relic is at the centre of the cluster (after Slee and Roy, 1998: L87).

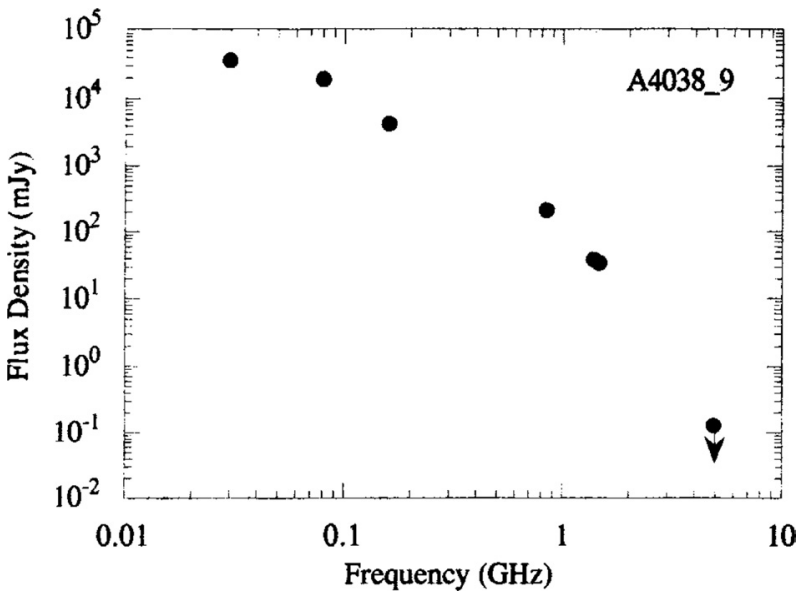

Figure 60 The radio spectrum of A4038_9, based on the 1997 VLA measures and other observations at earlier dates by Slee (after Slee and Roy, 1998: L90).
Slee's next contribution on clusters of galaxies took the above theme further by reporting on highest-resolution VLA observations of three new relic radio sources in clusters of galaxies. The observations at $1425 \mathrm{MHz}$ were made in 1998 June and September in Abell clusters A13, A85, A133 and A4038, and the paper reporting this work also included the A4038_9 source discussed above (which therefore will not be discussed here). Slee, Roy, Murgia, Andernach and Ehle (2001: 1173) described how the relics "... show a remarkable variety of fine structure that takes the form of arcs, filaments, and loops of enhanced surface brightness. Most of the arcs and filaments are barely resolved $(5 \mathrm{kpc})$ in their transverse directions, but they can have projected lengths of up to $100 \mathrm{kpc}$." A85 was the only relic that was highly polarised, but the levels varied throughout the source from 5\% up to $35 \%$, and the higher polarisation levels suggested the presence of highly ordered magnetic fields and low differential Faraday rotation. It was noted that the identification of optical host galaxies remained problematic in that the travel times from the relics in A85 and A133 to the nearest possible bright cluster galaxies were significantly longer than the modelled ages of the relics; however, there was always at least one relatively bright nearby elliptical galaxy that provided a better match. In the case of the A 85 and A133 relics there was slightly enhanced diffuse $\mathrm{X}$-ray emission in the region of each source, presumably from inverse Compton scattering, indicating magnetic field strengths of $0.47 \mu \mathrm{G}$ for $\mathrm{A} 85$ and $0.60 \mu \mathrm{G}$ for A133. Finally, Slee et al. (2001: 1172) found

“... excellent fits to the broadband radio spectra using the anisotropic model of spectral aging, and we have extended the model to include diffusion of particles between regions of different field strength. The steep radio spectra imply ages for the relics of $\sim 10^{8} \mathrm{yr}$, at the start of which period their radio luminosities would have been $\sim 10^{25} \mathrm{~W} \mathrm{~Hz}^{-1}$ at $1.4 \mathrm{GHz}$, and so their progenitors were on the boundary between FR I and FR II radio galaxies and hence among the most luminous $7 \%$ of radio galaxies. We find that the relics are in approximate pressure equilibrium with the surrounding intracluster gas, which has probably limited their free expansion and prevented them from fading by adiabatic cooling."

The most recent 'Slee' paper at the time of writing (2002 August) was a collaboration with seven international authors examining the X-ray characteristics of the cool core in the galaxy cluster A133 in the light of the radio observations published by Slee et al. (2001) above. Chandra X-Ray Observatory images showed that the core had a complex structure, the most prominent feature being a 'tongue' of emission that extended from the central $\mathrm{cD}$ galaxy to the north-west and partially overlapped the radio relic. In this context, the origin of the radio relics remained problematic in that it could be a radio source that was generated by a shock wave from merging clusters or a displaced radio lobe (or lobes) from the radio source associated with the central $\mathrm{cD}$ galaxy in the cluster 
(Fujita et al., 2002: 778). Although the assembled evidence was inconclusive, the authors tended to favour the second alternative.

Further research on clusters of radio galaxies and on relics is in progress.

\section{Discussion}

Apart from the length of time Slee has been actively involved in radio astronomical research, one of the other features of his work that immediately draws attention is his wide-ranging coverage. At one time or another he has addressed research problems that extend from our immediate terrestrial environment (the ionosphere), the solar outer corona, and the planets and comets of our solar system, through our Galaxy, with emphasis on its unusual stellar populations, the ISM, and some of its gaseous nebulae, to the galaxies, clusters of galaxies and quasars at the far reaches of the Universe. What is even more impressive is the extended intervals over which he has pursued

Table 1. The date range of Slee's publications in different research areas

\begin{tabular}{|c|c|c|c|}
\hline \multirow[t]{2}{*}{ Research area } & \multicolumn{2}{|c|}{ Date of publication } & \multirow{2}{*}{$\begin{array}{l}\text { Number of } \\
\text { publications }\end{array}$} \\
\hline & First & Last $^{\mathrm{A}}$ & \\
\hline 'Radio stars' & 1949 & 1955 & 10 \\
\hline The ionosphere \& IPM & 1953 & 1974 & 7 \\
\hline Fleurs survey & 1957 & 1963 & 8 \\
\hline Solar corona & 1956 & 1980 & 7 \\
\hline Jovian bursts & 1963 & 1968 & 6 \\
\hline Comet tails & 1957 & 1992 & 5 \\
\hline Flare stars & 1963 & $2001+$ & 16 \\
\hline Other active stars & 1982 & $2002+$ & 33 \\
\hline Pulsars & 1968 & 1987 & 8 \\
\hline Sagittarius A, etc. & 1954 & 1996 & 13 \\
\hline Interstellar medium & 1967 & 1988 & 10 \\
\hline Galaxies and LBI & 1965 & 1995 & 13 \\
\hline Culgoora surveys & 1972 & 1995 & 16 \\
\hline Clusters of galaxies & 1968 & $2002+$ & 14 \\
\hline Total & & & 166 \\
\hline
\end{tabular}

A The ' + ' indicates that research is continuing. research in some of these fields, and this is documented in Table 1 by listing the date range of his publications in each of the fields addressed in this paper. His longterm involvement (i.e. $\geq 20 \mathrm{yr}$ ) in all fields except the early 'radio stars', the Fleurs survey and Jovian bursts is apparent, but when the numbers of his publications are also taken into account, then his long-term commitment to flare stars, other active stars, long-baseline interferometry of radio galaxies, the Culgoora source survey, and clusters of galaxies is noteworthy.

This catholic approach to research is unusual, but says something of Slee's perseverance and commitment. When he was appointed to the Division of Radiophysics as a technical assistant in 1946 it was with an expectation of a career in electronics, and "... Joe Pawsey gave me the advice that this is the path I should follow — in other words, I could not reasonably aspire to a career on the research side of radio astronomy." (Slee, personal communication, 2002). However, reasonably early in the 'radio stars' work he came to realise that his real interest was in astronomical research, and this is partly what motivated him to complete a science degree and to seek appointment as a research scientist rather than remain a member of the technical staff. His personal ambition was to contribute in a broad range of astronomical areas, with emphasis on the observational and analytical aspects rather than on instrumentation development or theoretical issues, and he has continued to adhere to this policy even in retirement, and maintains an active observing program involving the Australia Telescope Compact Array (see Fig. 61) and the VLA.

Before moving on from Slee's fields of research interest, it is important that we mention that in addition to the afore-mentioned fields he has also been involved in two other areas of radio astronomy: instrumentation and history. It was his background as a radar engineer that brought him to Radiophysics in 1946, and even though he quickly gravitated to astronomical research — as we have seen - he also was actively involved in designing, building, testing, operating and maintaining items of radio astronomical hardware, from receivers, dipoles and feed

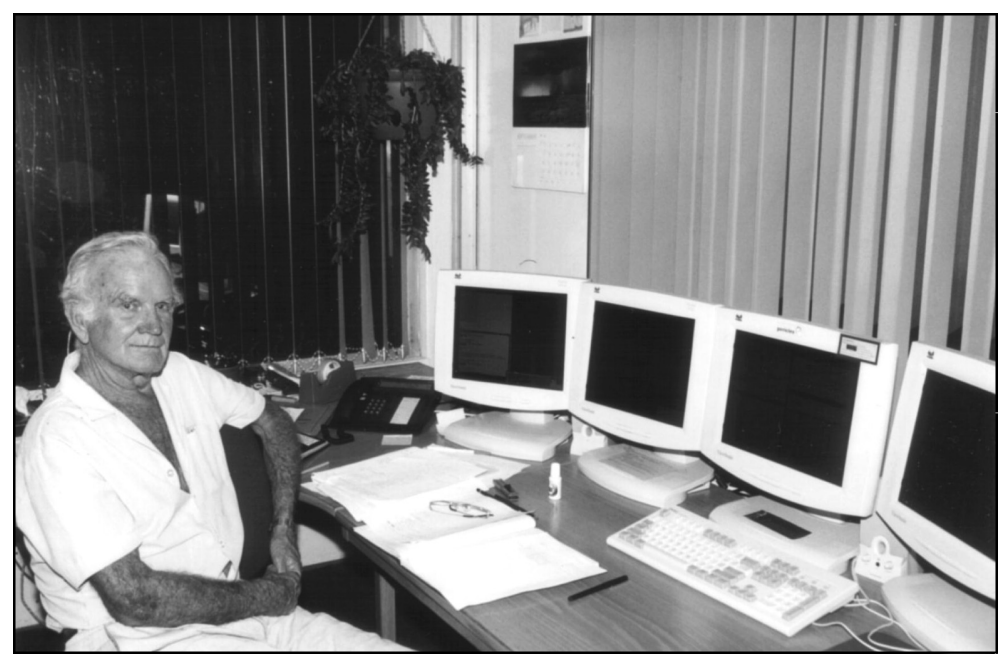

Figure 61 Bruce Slee at the control desk of the Australia Telescope Compact Array in 2002 September. 
horns to complete radio telescopes. Examples of this work would be the pioneering 3.9-m parabola at Dover Heights and, of course, the famous 'hole-in-the-ground' antenna, but he was also instrumental in maintaining and modifying the Mills and Shain Cross telescopes at Fleurs and setting up the various long-baseline interferometry systems that operated from this site. In this context, maintaining the equipment at the remote sites used in these projects was often a non-trivial task. Despite the time and effort that he put into the instrumentation side of research, Slee's name rarely appeared on papers about instrumentation. In fact, I could identify only four that focus exclusively or substantively on this area. One is a discussion of the principles of the sea interferometer - of Dover Heights fame - co-authored with John Bolton (Bolton and Slee, 1953); another is the basic technical account of the Mills Cross (Mills, Little, Sheridan and Slee, 1958); a third paper provides a description of the enigmatic 'barleysugar' antennas that he and Scheuer hoped to use to study source sizes (see Scheuer, Slee and Fryar, 1963); and the fourth and final paper deals with a novel noise-adding radiometer developed for the Parkes Radio Telescope (see Wright et al. 1986). However, many other papers by Slee contain technical information about the instrumentation used in his research programs.

The other 'missing' area of astronomy with which Slee has been involved is the history of our discipline. His first foray into this field was in 1994 when he drew on his personal recollections to contribute a fascinating paper about the Dover Heights field station for the special John Bolton issue of the Australian Journal of Physics (Slee, 1994). Much more recently, he has teamed with Orchiston and prepared a research paper on the Dover Heights 'hole-inthe-ground' antenna (Orchiston and Slee, 2002d), more popular articles about the Fleurs field station (Orchiston and Slee, 2002c) and his independent detection of solar radio emission in 1945 (Orchiston and Slee, 2002a), and an obituary for Gordon Stanley (Orchiston and Slee, 2002b). Other papers and articles are in preparation. Slee is now very much a part of Australian radio astronomical history, and perhaps in recognition of this reality he became a member of IAU Commission 41 (History of Astronomy) in 2002. As such, he joins a growing band of radio astronomers interested in documenting the history of our discipline and preserving its heritage.

If we add instrumentation and astronomical history to Slee's research portfolio, it is illuminating to identify the obvious 'missing links' - those major research areas with which he was never involved. Only two obvious contenders come to mind: solar burst emission and research on molecular lines. Both were strengths in the Radiophysics' arsenal, with research teams that quickly built up international reputations (e.g. see Christiansen, 1984; Robinson, 1994; Sullivan, 1988; Whiteoak, 1994). Circumstances determined that Slee would make contributions in other areas of radio astronomy.

Bolton arrived at Radiophysics just two months before Slee, and needed a technical assistant, a research project and a field station. Pawsey assigned him to Dover Heights, and assigned Slee to him. History quickly dictated that Bolton, Stanley and Slee would become international figures in 'radio star' research, thus channelling all three into the Cosmic Group (as opposed to the Solar Group) at Radiophysics.

As the junior partner of this famous trio, Slee initially had little opportunity - or desire - to dictate the direction of research, but as he gained maturity, experience and formal tertiary training in science he was able to develop his own research project (on radio star scintillations). Radio astronomy ceased at the Dover Heights field station in 1954, and at that time serious non-solar research was being conducted on just three other sites in the Sydney region: the long-established Potts Hill and Hornsby Valley field stations, and Fleurs, home to the brand new 85.5 MHz Mills Cross. Given Slee's source survey background and his experience in building and maintaining equipment that operated in the $60-100 \mathrm{MHz}$ range, it is no surprise that Pawsey decided that he should go to Fleurs, and continue to research discrete sources in collaboration with Mills. As we have seen, the team of Mills, Slee and Hill quickly gained international visibility as the new Mills Cross continued to yield ever-increasing numbers of sources.

It was while he was at Fleurs that Slee began to establish a degree of autonomy, and expand his research repertoire, so by the time of Mills' transfer to a chair at the University of Sydney and Shain's untimely passing (both in 1960) Slee was principal, or co-principal, investigator of projects investigating coronal scattering, the IPM, flare star emission, the sizes of discrete sources and the source(s) of the Jovian decametric emission. Some of this work drew him back to interferometry, a direction favoured by Bolton, Mills and Pawsey, amongst others, before the 'Giant Radio Telescope' (as the Parkes dish was originally known) won the day. In addition to his wide-ranging research portfolio, Slee also became known as the Division's leading exponent of long baseline interferometry (LBI).

Bolton returned to Australia in 1961 to take up the Directorship of the Parkes Radio Telescope, and he was keen to help mend the rift that had developed between Radiophysics and the British radio astronomers as a result of the infamous $2 C$ episode. And despite the prevailing focus on the Parkes parabola and the up-coming Culgoora Radioheliograph, he was also eager to make sure that Australia retained a knowledge of forefront LBI techniques. His solution was to negotiate with Hey, and establish a two-year contract appointment at the Royal Radar Establishment. When it came to a suitable Australian candidate, Slee was the obvious choice.

Slee returned to Sydney in 1966, and it took several years before a local VLBI facility in the form of the Parkes-Tidbinbilla Interferometer (and later the SHEVE Project) became a reality. In the interim, he chose to exploit the non-solar potential of the Culgoora Radioheliograph. This allowed him to continue working at 'low frequencies' (as $80 \mathrm{MHz}$, and ultimately $160 \mathrm{MHz}$, were 
Table 2. Slee's publications, by research journal and conference proceedings

\begin{tabular}{|c|c|}
\hline Publication's title & No. of papers ${ }^{\mathrm{A}}$ \\
\hline Astronomical and Astrophysical Transactions & 2 \\
\hline Astronomical Journal & 8 \\
\hline Astronomy and Astrophysics & 1 \\
\hline Astrophysical Journal & 12 \\
\hline Astrophysical Letters (later and Communications) & 6 \\
\hline ATNF News & 3 \\
\hline Australian Journal of Astronomy & 1 \\
\hline Australian Journal of Scientific Research and Australian Journal of Physics & 39 \\
\hline Journal of Astronomical History and Heritage & 1 \\
\hline Mitteilungen der Astronomische Gesellschaft & 1 \\
\hline Monthly Notices of the Royal Astronomical Society & 23 \\
\hline Nature & 15 \\
\hline New Astronomy & 1 \\
\hline Planetary and Space Science & 1 \\
\hline Proceedings (later Publications) of the Astronomical Society of Australia & 23 \\
\hline Proceedings of the Institute of Radio Engineers & 1 \\
\hline Proceedings of the Institute of Radio Engineers Australia & 1 \\
\hline Radio Science & 1 \\
\hline Sky and Telescope & 1 \\
\hline The Observatory & 3 \\
\hline Turkish Journal of Physics & 1 \\
\hline Papers in conference proceedings & 21 \\
\hline Total & 166 \\
\hline
\end{tabular}

perceived to be in those days), and to address a variety of research challenges (involving mainly flare stars, other chromospherically-active stars, the ISM, pulsars, radio galaxies and clusters of galaxies). Unlike some in Radiophysics who viewed Parkes as the sole non-solar instrument, this strategy particularly appealed to Bolton, who saw the obvious benefit of drawing on additional low-frequency data points when it came to plotting and interpreting source spectra.

However, Slee was not entirely wedded to the Radioheliograph, for between 1966 and its closure in 1984 he also made regular use of the Parkes Radio Telescope for multiwavelength observations at higher frequencies, and accelerated this access during 1984-1989, after which time the AT became readily available. His primary Parkes targets during these two decades were flare stars and other active stars, but the plasma tails of comets, interstellar scattering, compact galaxies and even pulsars also featured at one time or another.

Underlying Slee's research strategy was a burning desire to attack a variety of different research fields, using the best possible instrumentation available. Sometimes, it was a matter of realising new opportunities and exploiting these, as for example when the Culgoora Radioheliograph was employed for non-solar research. In more recent years he has also made a point of using international facilities, such as the VLA and various specialist orbiting astronomical observatories.

Local and international collaborations have been important to Slee, ever since the 1960s when he began formulating his own research projects on a regular basis.
Nowadays, papers featuring long lists of authors are not uncommon, but Slee was one of those Australian astronomers who pioneered wide-ranging international collaborations back in the 1970s and 1980s. Over the years, co-authors on his papers have included colleagues from Canada, England, Germany, Hungary, India, Italy, Japan, Mexico, Netherlands, New Zealand, Northern Ireland, Russia, Scotland, South Africa, Sweden, Taiwan and the United States, highlighting his desire to transcend geographical boundaries and political tensions in order to promote the true international spirit of scientific co-operation.

However, these comments should not blind us to the fact that half of Slee's collaborators were Australianbased, and exactly half of these were colleagues at Radiophysics or the ATNF. In areas where Slee was the obvious team leader, he was able to build up enduring, and in some cases sizable, research collaborations. For example, his flare star work involved 25 different Australian collaborators, six of whom were from Radiophysics, while the active stars project had 28 collaborators, nine from Radiophysics. And despite a perception by some that Slee's Culgoora work was carried out in virtual isolation, it is illuminating to note that of the seven Australian collaborators who participated in the source surveys five were from Radiophysics, while identical figures relate to his Culgoora clusters of galaxies project. In other words, Slee was able to build up a viable non-solar group at Culgoora, and to maintain this as a visible research entity throughout the life of the Radioheliograph. In examining Slee's overall Culgoora and Parkes research programs, 


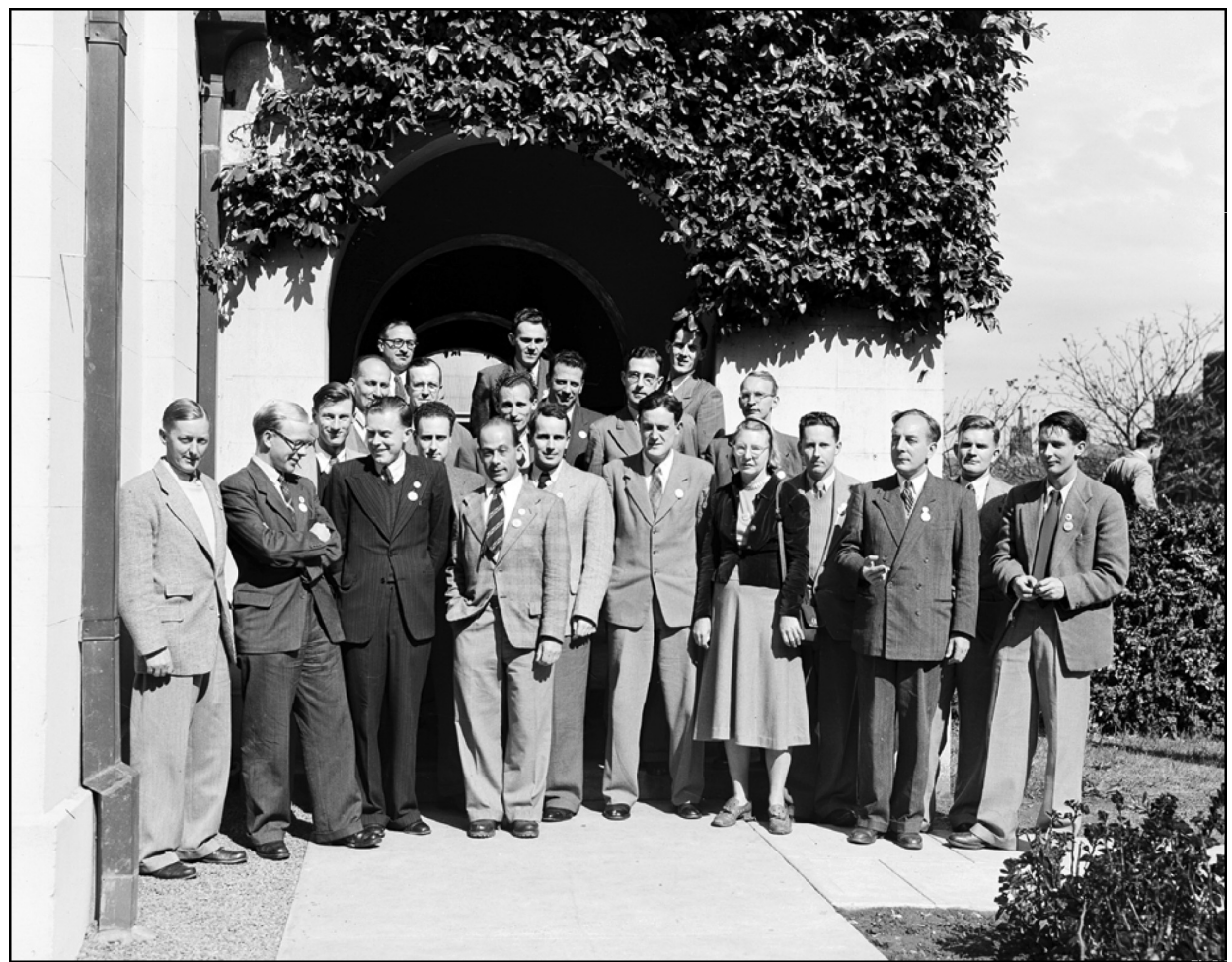

Figure 62 Group photograph showing some of the leading radio astronomers at the 1952 URSI General Assembly in Sydney. John Bolton and Bruce Slee are first and second from the right, respectively (ATNF Historic Photographic Archive: 2842-43).

it is significant that well respected colleagues like Dulk, Nelson, R. Robinson, Stewart, White and Wright tend to appear year after year as co-authors of papers penned by Slee.

Despite the relatively large number of international collaborators involved in Slee's research projects, not all of his papers were published in international journals - as Table 2 illustrates. In the Division of Radiophysics there was a policy of publishing major papers in the Australian Journal of Scientific Research and one of its successors, the Australian Journal of Physics, and as a result of such a policy this omnibus Australian physics journal gained international visibility because of Australia's rapid emergence as a leading player in international radio astronomy (see Sullivan, 1988: 328). At a later date, when the Astronomical Society of Australia (ASA) was founded, staff members were also encouraged to publish papers - or at least preliminary reports - in its Proceedings and later in its Publications, hence the comparatively large number of relevant entries in Table 2. Likewise, during the 1940s and 1950s, staff were also encouraged to gain an instant international readership for their most important research findings by publishing preliminary accounts in Nature, with the obligatory papers of substance appearing elsewhere (typically in the Australian Journal of Physics or Monthly Notices of the Royal Astronomical Society) at a later date.

Although a member of the IAU and the ASA, Slee has always preferred to devote his time and energy to research rather than administration or astronomical politics, and as a consequence he has not been actively involved in the organisation of these or other astronomical societies. An additional contributing factor in this context has been his shy nature, which also goes some way towards explaining his rather infrequent attendance at international conferences. Having said that, one international conference he thoroughly enjoyed was the URSI General Assembly, which was held in Sydney in 1952 (see Kerr, 1953). This was the first time that any of the international scientific unions had met outside Europe or the USA, and despite the general inaccessibility of Australia at the time - for a lengthy passage by ship was involved - it drew many of the world's leading radio astronomers. The scheduling of this event in Sydney was formal recognition of Australia's proud reputation in radio astronomy, and Slee's contributions at Dover Heights were a contributory factor. He features in a group photograph (see Fig. 62) that was taken on August 8, just two days before his 28th birthday, alongside such overseas notables as H. I. Ewen, J. P. Hagen, R. Hanbury Brown, C. A. Muller, F. G. Smith and J. L. Steinberg. Also in the photograph are Australian colleagues, J. G. Bolton, W. N. (Chris) Christiansen, E. R. Hill, J. V. Hindman, F. J. Kerr, A. G. Little, B. Y. Mills, R. Payne-Scott, J. H. Piddington, C. A. Shain, S. F. Smerd and J. P. Wild, all well known names in the history of Australian radio astronomy.

Despite his quiet unassuming nature, over the years Slee has succeeded in reaping rewards - other than personal satisfaction - for his research output. In addition to receiving a D.Sc. degree from the University of New South Wales, he is a Fellow of the Astronomical Society of Australia, an honour shared by only about one-fifth of the 


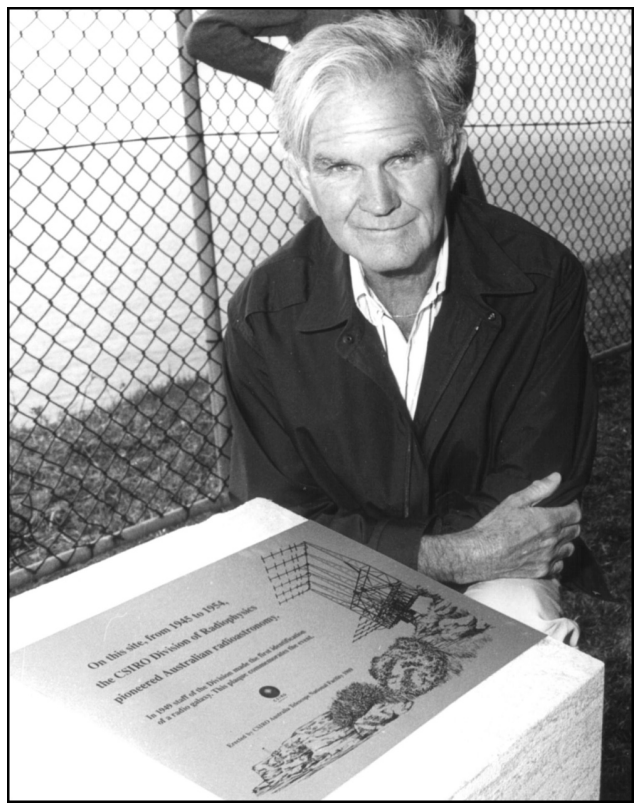

Figure 63 Bruce Slee posing beside the commemorative plaque at Dover Heights in 1989 November (adapted from ATNF Historic Photographic Archive: 15506).

membership, and in 1989 November the pioneering role that he, Bolton and Stanley played at Dover Heights during the 1940s and early 1950s was recognised when a plaque was unveiled commemorating the amazing contribution that this field station made to Australian and international radio astronomy (see Fig. 63). This plaque is still on display, but a visit to the Dover Heights site brings a sobering reflection that of the many antennas Slee used during his first two decades at Radiophysics the only one that has survived to the present day - and continues to function efficiently — is the 64-m Parkes Radio Telescope. All that remains of the Dover Heights field station is the rusting remains of the $6 \times 2$-Yagi array mounting, some concrete rubble from the blockhouse, and a recently-installed historical display and replica of the old $4 \times 2$-Yagi antenna, while a visit to Fleurs reveals that the novel Mills Cross and Shain Cross antennas are no more, having long ago rotted, rusted and disintegrated. Thus, to track Slee's initial exploits in radio astronomy is to explore the early history of these Radiophysics field stations and to mourn the loss of so much of our pioneering radio astronomical heritage. We can but hope that reason will prevail and that those early radio telescopes that have survived, including the 18-m Kennedy parabola at Parkes, parts of the Chris Cross and the Fleurs Synthesis Telescope at Fleurs, and the Radioheliograph and Radiospectrograph at Culgoora, will be restored and preserved for posterity.

\section{Concluding Remarks}

Bruce Slee is one of the few surviving founders of Australian radio astronomy, and although now long retired is the only one who continues with an active research program. He regularly applies for and is granted observing time on the Australia Telescope Compact Array in order to investigate the properties of different types of active stars, and in collaboration with US-based colleagues uses the VLA in a bid to understand the radio properties of clusters of galaxies and those enigmatic cluster 'relics'.

These are but two fields among the wide-ranging research portfolio that Slee has actively pursued for more than half a century. Along with Bolton and Stanley he solved the mystery of the 'radio stars', and showed optical astronomers that radio astronomy had an important role to play in astronomical investigations. His Dover Heights studies also revealed that source variations were caused by the Earth's ionosphere and were not intrinsic to the sources themselves. Once at Fleurs, Slee, Mills and Hill completed one of the most extensive source surveys carried out up to that time, in the process revealing problems inherent in its northern competitor, the Cambridge $2 C$ catalogue. They showed that some of the sources in the MSH catalogue had optical correlates, either gaseous nebulae in our Galaxy or extragalactic nebulae, and forecast that many of the latter would prove to be radio emitters. They also produced the first detailed isophote map of the Galactic plane at metre wavelengths.

While at Fleurs, Slee also turned his attention back to our immediate celestial neighborhood, using occultations of selected discrete sources to investigate electron density inhomogeneities in the solar corona out to distances of $\sim 60$ solar radii. However, sporadic increases in coronal scattering out to 100 solar radii were also recorded, and he was able to document irregularities in the IPM at distances of 1-4 a.u. from the Sun. Slee researched the source of the Jovian decametric burst emission, setting an upper limit to the angular size of the emitting region. He and Gent also discovered a new type of sub-millisecond Jovian decametric burst. Whilst still focussing on the Solar System, Slee and various collaborators provided new data on electron density variations in the plasma tails of several different comets, showing the existence of small-scale turbulence nearer to the cometary nucleus and large-scale turbulence at the transition region between the tail and the solar wind.

One of Slee's greatest contributions has been to our understanding of flare stars and other types of active stars. During the early 1960s, he and Lovell were the first to document the existence of radio emission from flare stars, and Slee's subsequent research has provided valuable information about radio flares associated with several different flare stars. As part of this work, he was involved in the very first multi-wavelength flare star campaign, when coordinated X-ray, optical and radio observations of YZ Canis Minoris were carried out in 1975. He was also one of the first to search successfully for radio emission from other types of active stars, and for more than two decades has continued to make major contributions in this field. Through his efforts, quiescent and/or outburst radio emission has been recorded from Algol, AM Her, Am, BY Draconis, F supergiant, FK Comae, RS CVn, red giant, dwarf pre-main sequence and unspecified Ca II emitting types of stars. Studies of these stars and the classical dMe flare stars have provided information on 
radio luminosities, spectra, polarisation, coronal electron densities and magnetic field strengths, and the generating mechanisms associated with the radio emission. This is one of the fields in which he is continuing to make contributions.

For several years Slee researched pulsars, and provided new light on pulse widths, fluxes and amplitude variations. His Galactic studies produced the earliest isophote maps of the Galactic Centre, and subsequent collaborative studies provided increasing detail of the Sgr A source as access was gained to ever more sophisticated instrumentation. He also conducted one of the first detailed surveys of radio emission from planetary nebulae. Over more than two decades, he carried out studies of the ISM, using mainly radio emission from supernovae and pulsars, and was able to relate the derived electron densities and pattern scale sizes over various path lengths to different models of scattering in vogue at the time.

Soon after returning from England, Slee used the Culgoora Radioheliograph to carry out a comprehensive sky survey and over a ten-year period recorded 2167 sources at $80 \mathrm{MHz}$ and 2045 sources at $160 \mathrm{MHz}$. In addition to investigating positions, flux densities, angular sizes and source structure, he studied the spectra of $\sim 2000$ of these sources — at the time the largest analysis of this kind ever undertaken - and showed that different types of galaxies were associated with significantly different spectra. He also investigated the relationship between spectral slope and galaxy redshift, and showed that between 1972 and 1984 nearly $50 \%$ of all galaxies displayed variations in radio flux on time intervals of months or years. Of particular interest, in this regard, were the metre-wave 'outbursts' characteristic of the QSO, 1055+018.

After his experience with LBI in England during 19651966, it is no surprise that Slee employed this technique in Australia during the 1980s and early 1990s. He and colleagues in the SHEVE consortium studied the milliarcsecond features of Cen A, QSOs and radio galaxies, and later he used the Parkes-Tidbinbilla Interferometer to provide new information on the fine-scale angular structure, spectra, and emission mechanisms of the radio-continuum cores of different types of galaxies, including Seyferts.

The final area of astronomy in which Slee has made a significant contribution is in unravelling the radio properties of clusters of galaxies. After an initial project involving 353 different Abell clusters and the Culgoora Radioheliograph and Parkes Radio Telescope, he commenced the 'VLA Survey of Rich Clusters of Galaxies', and between 1981 and 1985 examined 58 different cluster fields. Valuable data were assembled on the radio power, morphologies, flux densities, spectra, optical identifications and relation to X-ray luminosity of the detected sources. In the course of this survey, Slee detected and investigated several steep-spectrum 'relics'. His research into clusters of galaxies and 'relics' is continuing.

Owen Bruce Slee is a pioneering Australian radio astronomer, and it is a testimony to his dedication and his passion for astronomy that after more than half a century he continues an active research program. Since joining the CSIRO's Division of Radiophysics in 1946 he has made an important, long-term, wide-ranging contribution to astronomy and to Australian science, and his work deserves to be better known and recognised. It is to be hoped that this paper will go some way towards achieving this.

\section{Acknowledgements}

I should like to thank Dick Manchester for making this biographical study possible and for commenting on the manuscript; an anonymous referee for his helpful comments; and the following colleagues for their assistance: Jim Caswell, Jessica Chapman and Bob Sault (all ATNF), Stuart Ryder (AAO), and staff from the Radiophysics Laboratory Library. Finally, I am particularly grateful to Bruce Slee for his friendship, guidance and encouragement, and to the ATNF for permission to publish images drawn from the ATNF Historic Photographic Archive.

\section{References}

Ables, J. G., Komesaroff, M. M., \& Hamilton, P. A. 1970, Ap. Letters, 6, 147

Adgie, R. L., et al. 1965, Nature, 208, 275

Alurkar, S. K., Slee, O. B., \& Bobra, A. D. 1986, Aust. J. Phys., 39,433

Andernach, H., Gubanov, A. G., \& Slee, O. B. 1998, in Observational Cosmology with the New Radio Surveys, eds. M. Bremer, N. Jackson, \& I. Perez-Fournon (Dordrecht: Kluwer), 104

Artyukh, V. S., Kuz'min, A. D., \& Makarov, A. N. 1972, Soviet Astron., AJ, 16, 372

Baade, W., \& Minkowski, R. 1954, ApJ, 119, 20

Bastian, T. S., Dulk, G. A., \& Slee, O. B. 1988, AJ, 95, 794

Beasley, A. J., Ball, L. T., Budding, E., Slee, O. B., \& Stewart, R. T. 1993, AJ, 106, 1656

Biraud, F., et al. 1974, A\&A, 34, 163

Bolton, J. G. 1948, Nature, 162, 141

Bolton, J. G. 1955, Vistas in Astron., 1, 568

Bolton, J. G. 1982, PASA, 4, 349

Bolton, J. G., \& Slee, O. B. 1953, Aust. J. Phys., 6, 420

Bolton, J. G., \& Slee, O. B. 1957, in Radio Astronomy, ed. H. C. van de Hulst (Cambridge: CUP), 174

Bolton, J. G., \& Stanley, G. J. 1949, Aust. J. Sci. Res., A2, 139

Bolton, J. G., Stanley, G. J., \& Slee, O. B. 1949, Nature, 164, 101

Bolton, J. G., Stanley, G. J., \& Slee, O. B. 1953, Aust. J. Phys., 6,434

Bolton, J. G., Stanley, G. J., \& Slee, O. B. 1954, Aust. J. Phys., 7,110

Bolton, J. G., \& Westfold, K. C. 1950, Aust. J. Sci. Res., A3, 19

Bolton, J. G., Westfold, K. C., Stanley, G. J., \& Slee, O. B. 1954, Aust. J. Phys., 7, 96

Budding, E., Burgess, A., Chan, S., \& Slee, O. B. 1991, in Surface Inhomogeneities in Late-type Stars, eds. P. B. Byrne, \& D. J. Mullan (Armagh: Armagh Observatory), 253

Budding, E., Jones, K. L., Slee, O. B., \& Watson, L. 1999a, MNRAS, 305,966

Budding, E., Jones, K. L., Slee, O. B., \& Watson, L. 1999b, Turkish J. Phys., 23, 453

Budding, E., Lim, J., Slee, O. B., \& White, S. M. 2002, New Astron., 7, 35

Budding, E., Marsden, S. C., \& Slee, O. B. 2001, PASA, 18, 140

Budding, E., Slee, O. B., \& Jones, K. 1998, PASA, 15, 183

Budding, E., Slee, O. B., \& Stewart, R. T. 1992, in Evolutionary Processes in Interacting Binary Stars, eds. Y. Kondo, R. F. Sisteró, \& R. S. Polidan (Dordrecht: Kluwer), 415 
Budding, E., et al. 1997, in The Third Pacific Rim Conference on Recent Developments in Binary Star Research, ed. K.-C. Leung (San Francisco: ASP), 255

Bunton, J. D., et al. 1986, PASA, 6, 316

Bunton, J. D., et al. 1989, PASA, 8, 127

Christiansen, W. N. 1984, in The Early Years of Radio Astronomy, ed. W. T. Sullivan (Cambridge: CUP), 113

Cole, T. W., \& Slee, O. B. 1980, Nature, 285, 93

Collier, A. C., Haynes, R. F., Slee, O. B., Wright, A. E., \& Hillier, D. J. 1982, MNRAS, 200, 869

Dagkesamansky, R. D., Gubanov, A. G., Kuzmin, A. D., \& Slee, O. B. 1982, MNRAS, 200, 971

De Pater, I., \& Butler, B., submitted to Icarus

Dulk, G. A., \& Slee, O. B. 1972, Aust. J. Phys., 25, 429

Dulk, G. A., \& Slee, O. B. 1974, Nature, 248, 33

Dulk, G. A., \& Slee, O. B. 1975, ApJ, 199, 61

Ekers, R. D., van Gorkom, J. H., Schwarz, U. J., \& Goss, W. M. 1983, A\&A, 122, 143

Fujita, Y., et al. 2002, ApJ, 575, 764

Gatt, J. A., \& Lyne, A. G. 1972, MNRAS, 158, 281

Gleeson, L. J., Legg, M. P. C., \& Westfold, K. C. 1970, PASA, 1,320

Goddard, B. R., Watkinson, A., \& Mills, B. Y. 1960, Aust. J. Phys., 13,665

Goddard, D. E., \& Haynes, R. F. (eds.) 1994, Pioneering a New Astronomy. Papers in Memory of John G. Bolton. Aust. J. Phys., 47(5)

Goddard, D. E., \& Milne, D. K. (eds.) 1994, Parkes. Thirty Years of Radio Astronomy (Melbourne: CSIRO Publishing)

Haisch, B. M., et al. 1978, ApJ, 225, 35L

Haisch, B. M., et al. 1981, ApJ, 245, 1009

Haynes, R., Haynes, R., Malin, D., \& McGee, R. (eds.) 1996, Explorers of the Southern Sky. A History of Australian Astronomy (Cambridge: CUP)

Hewish, A. 1980, Nature, 285, 95

Hey, J. S., Phillips, J. W., \& Parsons, S. J. 1946, Nature, 157, 296

Higgins, C. S., Komesaroff, M. M., \& Slee, O. B. 1971, Ap. Letters, 9,75

Hill, E. R., Slee, O. B., \& Mills, B. Y. 1958, Aust. J. Phys., 11, 530

Huchtmeier, W. K., \& Slee, O. B. 1976, MitAG, 38, 87

Innis, J. L., et al. 1985, PASA, 6, 160

Janardhan, P., Alurkar, S. K., Bobra, A. D., \& Slee, O. B. 1991, Aust. J. Phys., 44, 565

Janardhan, P., Alurkar, S. K., Bobra, A. D., Slee, O. B., \& Waldron, D. 1992, Aust. J. Phys., 45, 115

Jauncey, D. L., et al. 1989, AJ, 98, 44

Jones, K. L., Brown, A., Stewart, R. T., \& Slee, O. B. 1996, MNRAS, 283,1331

Jones, K. L., Robinson, R. D., Slee, O. B., \& Stewart, R. T. 1992, MNRAS, 256, 535

Karpen, J. T., et al. 1977, ApJ, 216, 479

Kellermann, K. 1966, Icarus, 5, 478

Kellermann, K., \& Sheets, B. (eds.) 1983, Serendipitous Discoveries in Radio Astronomy (Green Bank: National Radio Astronomy Observatory)

Kerr, F. J. 1953, Sky \& Telescope, 12(3), 59

Komesaroff, M. M., Slee, O. B., \& Higgins, C. S. 1968, PASA, 1,102

Lang, K. R., \& Rickett, B. J. 1970, Nature, 225, 528

Lim, J., Slee, O. B., \& Stauffer, J. R. 1996a, PASA, 1, 102

Lim, J., Slee, O. B., \& Stauffer, J. R. 1996b, in Cool Stars, Stellar Systems, and the Sun, eds. R. Pallavicini, \& A. K. Dupree (San Francisco: ASP), 371

Lim, J., White, S. M., \& Slee, O. B. 1996, ApJ, 460, 976

McGee, R. X., Slee, O. B., \& Stanley, G. J. 1955, Aust. J. Phys., 8,347

Malofeev, V. M., Malov, O. I., \& Shchegoleva, N. V. 2000, Astro. Repts, 44, 436

Manchester, D. 1994, in Goddard and Milne, 69
Meier, D. L., et al. 1988, in The Impact of VLBI on Astrophysics and Geophysics, eds. M. J. Reid, \& J. M. Moran (Dordrecht: Kluwer), 137

Meier, D. L., et al. 1989, AJ, 98, 27

Melrose, D. B. 1980, Plasma Astrophysics: Thermal Processes in Diffuse Magnetized Plasmas (two vols.) (New York: Gordon \& Breach)

Mills, B. Y. 1984, in The Early Years of Radio Astronomy, ed. W. T. Sullivan (Cambridge: CUP), 147

Mills, B. Y., Hill, E. R., \& Slee, O. B. 1958, The Observatory, 78, 116

Mills, B. Y., \& Little, A. G. 1953, Aust. J. Phys., 6, 272

Mills, B. Y., Little, A. G., \& Sheridan, K. V. 1956, Aust. J. Phys., 9, 218

Mills, B. Y., Little, A. G., Sheridan, K. V. \& Slee, O. B. 1958, Proc. Inst. Radio Eng., 46, 67

Mills, B. Y., \& Slee, O. B. 1957, Aust. J. Phys., 10, 162

Mills, B. Y., Slee, O. B., \& Hill, E. R. 1958, Aust. J. Phys., 11, 360

Mills, B. Y., Slee, O. B., \& Hill, E. R. 1960, Aust. J. Phys., 13, 676

Mills, B. Y., Slee, O. B., \& Hill, E. R. 1961, Aust. J. Phys., 14, 497

Morris, D., Schwarz, U. T., \& Slee, O. B. 1970, Ap. Letters, 5, 187

Nelson, G. J., et al. 1979, MNRAS, 187, 405

Nelson, G. J., et al. 1986, MNRAS, 220, 91

Orchiston, W. 1993, Southern Stars, 35, 46

Orchiston, W. 1994a, in Goddard \& Haynes, 541

Orchiston, W. 1994b, in Carter Observatory Handbook for 1995 , eds. W. Orchiston, R. Dodd, \& R. Hall (Wellington: Carter Observatory), 65

Orchiston, W., \& Slee, B. 2002a, AAO Newsl., 101, 25

Orchiston, W., \& Slee, B. 2002b, ATNF News, 46, 3

Orchiston, W., \& Slee, B. 2002c, ATNF News, 47, 12

Orchiston, W., \& Slee, B. 2002d, J. Astron. History \& Heritage, 5, 21

Palmer, H. P., et al. 1967, Nature, 213, 789

Pawsey, J. L. 1946, Nature, 158, 633

Preston, R. A., et al. 1989, AJ, 98, 1

Rao, A. P., \& Slee, O.B. 1988, MNRAS, 234, 853

Robertson, P. 1992, Beyond Southern Skies. Radio Astronomy and the Parkes Telescope (Cambridge: CUP)

Robinson, B. 1994, in Goddard \& Milne, 106

Robinson, R. D., \& Slee, O. B. 1986, Aust. J. Astron., 1, 105

Robinson, R. D., Slee, O. B., \& Little, A. G., 1976, ApJ, 203, 91L

Robinson, R. D., Airapetian, V., Slee, O. B., Mathioudakis, M. \& Carpenter, K. G. 2001, in 11th Cambridge Workshop on Cool Stars, Stellar Systems, and the Sun, eds. R.J. Garcia-Lopez, R. Rebolo, \& M. R. Z. Osorio (San Francisco: ASP), 1151

Robinson, R. D., Carpenter, K. G., Slee, O. B., Nelson, G. J., \& Stewart, R. T. 1994, MNRAS, 267, 918

Sadler, E. M., Slee, O. B., Reynolds, J. E., \& Ekers, R. D. 1994, in The First Stromlo Symposium: The Physics of Active Galaxies, eds. G. V. Bicknell, M. A. Dopita, \& P. J. Quinn (San Francisco: ASP), 335

Sadler, E. M., Slee, O. B., Reynolds, J. E., \& Roy, A. L. 1995, MNRAS, 276, 1373

Seaquist, E. R., Gilmore, W., Nelson, G. J., Payten, W. J., \& Slee, O. B. 1980, ApJ, 241, L77

Scheuer, P. A. G., Slee, O. B., \& Fryar, C. F. 1963, Proc. Inst. Radio Eng. Aust., 24, 185

Schott, E. 1947, Phys. Blatt., 3, 159

Shain, C. A., \& Slee, O. B. 1957, The Observatory, 77, 204

Shakeshaft, J. R., Ryle, M., Baldwin, J. E., Elsmore, B., \& Thomson, J. H. 1955, MRAS, 67, 106

Shapirovskaya, N., Slee, O. B., \& Tsarevsky, G. S. 1995, A\&AT, 7,47

Sheridan, K. V., Labrum, N. R., \& Payten, W. J. 1973, Proc. Inst. Elect. \& Electron. Eng., 61, 1312

Slee, O. B. 1955, Aust. J. Phys., 8, 498

Slee, O. B. 1956, The Observatory, 76, 228

Slee, O. B. 1958, Nature, 181, 1610

Slee, O. B. 1959, Aust. J. Phys., 12, 134

Slee, O. B. 1961, MNRAS, 123, 223 
Slee, O. B. 1962, Aust. J. Phys., 15, 568

Slee, O. B. 1964, ApJ, 140, 823

Slee, O. B. 1966, Planet. \& Space Sci., 14, 255

Slee, O. B. 1967, in Radio astronomy and the Galactic System, ed. H. van Woerden (London: Academic Press), 237

Slee, O. B. 1969, PASA, 1, 223

Slee, O. B. 1972a, Ap. Letters, 12, 75

Slee, O. B. 1972b, PASA, 2, 159

Slee, O. B. 1977, Aust. J. Phys. Astrophys. Suppl. Number, 43, 1 Slee, O. B. 1981, PASA, 4, 184

Slee, O. B. 1982, in Extragalactic Radio Sources, eds. D. S. Heeschen, \& C. M. Wade (Dordrecht: Reidel), 35

Slee, O. B. 1984, MNRAS, 209, 215

Slee, O. B. 1994, in Goddard \& Haynes, 517

Slee, O. B. 1995, Aust. J. Phys., 48, 143

Slee, O. B., Allen, W. H., Coates, D. W., Page, A. A., \& Quinn, P. Q. 1984, in Proceedings of the Second Asian-Pacific Regional Meeting on Astronomy Bandung, Indonesia - 24 to 29 August 1981, eds. B. Hidayat, \& M.W. Feast (Jakarta: Tira Pustaka), 74

Slee, O. B., Alurkar, S. K., \& Bobra, A. D. 1986, Aust. J. Phys., 39,103

Slee, O. B., Bobra, A. D., \& Alurkar, S. K. 1987, Aust. J. Phys., 40,557

Slee, O. B., Bobra, A. D., Waldron, D., \& Lim, J. 1990, Aust. J. Phys., 43, 801

Slee, O. B., \& Budding, E. 1995, MNRAS, 277, 1063

Slee, O. B., \& Dulk, G. A. 1972, Aust. J. Phys., 25, 103

Slee, O. B., \& Dulk, G. A. 1974, in Galactic Radio Astronomy, eds. F. J. Kerr, \& S. C. Simonson (Dordrecht: Reidel), 347

Slee, O. B., Dulk, G. A., \& Otrupcek, R. E. 1980, PASA, 4, 100

Slee, O. B., \& Gent, H. 1967, Nature, 216, 235

Slee, O. B., Haynes, R. F., \& Wright, A. E. 1984, MNRAS, 208, 865

Slee, O. B., \& Higgins, C. S. 1963, Nature, 197, 781

Slee, O. B., \& Higgins, C. S. 1966, Aust. J. Phys., 19, 167

Slee, O. B., \& Higgins, C. S. 1968, Aust. J. Phys., 21, 341

Slee, O. B., \& Higgins, C. S. 1971a, Aust. J. Phys., 24, 247

Slee, O. B., \& Higgins, C. S. 1971b, Nature, 234, 210

Slee, O. B., \& Higgins, C. S. 1973, Aust. J. Phys., Astrophys. Suppl. Number, 27, 1

Slee, O. B., \& Higgins, C. S. 1975, Aust. J. Phys., Astrophys. Suppl. Number, 36, 1

Slee, O. B., Higgins, C. S., \& Patston, G. E. 1963, Sky \& Telescope, $25(2), 83$

Slee, O. B., Higgins, C. S., Roslund, C., \& Lyngå, G. 1969, Nature, 224,1087

Slee, O. B., \& Hill, E. R. 1971, Aust. J. Phys., 24, 441

Slee, O. B., Komesaroff, M. M., \& McCulloch, P. M. 1968, Nature, 219,342

Slee, O. B., \& Lee, P. Y. 1974, Aust. J. Phys., 27, 837

Slee, O. B., McConnell, D., Lim, J., \& Bobra, A. D. 1987, Nature, 325,699

Slee, O. B., \& Mulhall, P. S. 1970, PASA, 1, 322

Slee, O. B., \& Mulhall, P. S. 1971, Ap. Letters, 8, 5

Slee, O. B., \& Orchiston, D. W. 1965, Aust. J. Phys., 18, 187

Slee, O. B., \& Page, A. A. 1979, in Changing Trends in Variable Star Research, eds. F. M. Bateson, J. Smak, \& I. H. Urch (Hamilton: University of Waikato), 150

Slee, O. B., Perley, R. A., \& Siegman, B. C. 1989, Aust. J. Phys., 42,633

Slee, O. B., \& Quinn, P. J. 1979, PASA, 3, 332

Slee, O. B., \& Reynolds, J. E. 1984, PASA, 5, 516

Slee, O. B., \& Roy, A. L. 1998, MNRAS, 297, 86L

Slee, O. B., Roy, A. L., \& Andernach, H. 1996, Aust. J. Phys., 49, 977
Slee, O. B., Roy, A. L., \& Andernach, H. 1998, Aust. J. Phys., 51,971

Slee, O. B., Roy, A. L., Murgia, M., Andernach, H., \& Ehle, M. 2001, AJ, 122, 1172

Slee, O. B., Roy, A. L., \& Savage, A. 1994, Aust. J. Phys., 47, 145

Slee, O. B., Sadler, E. M., Reynolds, J. E., \& Ekers, R. D. 1994 MNRAS, 269, 928

Slee, O. B., \& Sheridan, K. V. 1975, PASA, 2, 336

Slee, O. B., Sheridan, K. V., Dulk, G. A., \& Little, A. G. 1983, PASA, 5,247

Slee, O. B., \& Siegman, B. C. 1983, PASA, 5, 114

Slee, O. B., \& Siegman, B. C. 1988, MNRAS, 235, 1313

Slee, O. B., Siegman, B. C., \& Mulhall, B. C. 1982, PASA, 4, 278

Slee, O. B., Solomon, L. H., \& Patston, G. E. 1963, Nature, 199, 991

Slee, O. B., \& Stewart, R. T. 1989, MNRAS, 236, 129

Slee, O. B., Tuohy, I. R., Nelson, G. J., \& Rennie, C. J. 1981, Nature, 292,220

Slee, O. B., White, G. L., Reynolds, J. E., \& Caganoff, S. 1990, Ap. Letters \& Comm., 27, 361

Slee, O. B., Wilson, I. R. G., \& Siegman, B. C. 1982, PASA, 4, 431

Slee, O. B., Wilson, I. R. G., \& Siegman, B. C. 1983, Aust. J. Phys., 36,101

Slee, O. B., \& Wraith, P. K. 1967, Nature, 214, 971

Slee, O. B., et al. 1974, MNRAS, 167, 31

Slee, O. B., et al. 1986, PASA, 6, 312

Slee, O. B., et al. 1987a, MNRAS, 227, 467

Slee, O. B., et al. 1987b, MNRAS, 229, 659

Slee, O. B., et al. 1987c, PASA, 7, 55

Slee, O. B., et al. 1988, Ap. Letters \& Comm., 27, 247

Slee, O. B., et al. 1989a, MNRAS, 239, 913

Slee, O. B., et al. 1989b, PASA, 8, 158

Smerd, S. F., \& Slee, O. B. 1966, Aust. J. Phys, 19, 427

Smith, A. G., \& Carr, T. D. 1964, Radio Exploration of the Planetary System (Princeston: Van Nostrand)

Stanley, G. J. 1994, in Goddard \& Haynes, 507

Stanley, G. J., \& Slee, O. B. 1950, Aust. J. Sci. Res., A3, 234

Stewart, R. T., Innis, J. L., Slee, O. B., Nelson, G. J., \& Wright, A. E. 1988, AJ, 96, 371

Stewart, R. T., et al. 1987, in Cool Stars, Stellar Systems, and the Sun. Proceedings of the Fifth Cambridge Workshop, eds. J. L. Linsky, \& R. E. Stencil (Berlin: Springer-Verlag), 95

Stewart, R. T., et al. 1989, ApJ, 342, 463

Sullivan, W. T. 1982, Classics in Radio Astronomy (Dordrecht: Reidel)

Sullivan, W. T. 1984, The Early Years of Radio Astronomy (Cambridge: CUP)

Sullivan, W. T. 1988, in Australian Science in the Making, ed. R. Home (Cambridge: CUP), 308

Turner, B. E. 1974, ApJ, 189, 137L

Tzioumis, A. K., et al. 1989, AJ, 98, 36

Vilhu, O., et al. 1993, A\&A, 278, 467

Whiteoak, J. B. 1994, in Goddard \& Milne, 75

Whitfield, G. R., \& Hogböm, J. 1957, Nature, 180, 602

Wild, J. P. 1967, Proc. Inst. Radio \& Electron. Engin. Aust., 28,279

Wright, A. E., Cropper, M., Stewart, R. T., Nelson, G. J., \& Slee, O. B. 1988, MNRAS, 231, 319

Wright, A. E., et al. 1986, PASA, 6, 512

Wright, A. E., et al. 1987, PASA, 7, 159

Yusef-Zadeh, F., Morris, M., Slee, O. B., \& Nelson, G. J. 1986a, ApJ, 300, 47L

Yusef-Zadeh, F., Morris, M., Slee, O. B., \& Nelson, G. J. 1986b, ApJ, 310, 689

1955, Nature, 175, 1074 